\title{
AN ADAPTIVE UNSCENTED KALMAN FILTER FOR TIGHTLY-COUPLED INS/GPS INTEGRATION
}

\author{
A THESIS SUBMITTED TO \\ THE GRADUATE SCHOOL OF NATURAL AND APPLIED SCIENCES \\ $\mathrm{OF}$ \\ MIDDLE EAST TECHNICAL UNIVERSITY
}

BY

TAMER AKÇA

IN PARTIAL FULLFILLMENT OF THE REQUIREMENTS

FOR

THE DEGREE OF MASTER OF SCIENCE

IN

ELECTRICAL AND ELECTRONICS ENGINEERING

FEBRUARY 2012 
Approval of the thesis:

\section{AN ADAPTIVE UNSCENTED KALMAN FILTER FOR TIGHTLY-COUPLED INS/GPS INTEGRATION}

submitted by TAMER AKÇA in partial fulfillment of the requirements for the degree of Master of Science in Electrical and Electronics Engineering Department, Middle East Technical University by,

Prof. Dr. Canan Özgen

Dean, Graduate School of Natural and Applied Sciences

Prof. Dr. İsmet Erkmen

Head of Department, Electrical and Electronics Engineering

Prof. Dr. Mübeccel Demirekler

Supervisor, Electrical and Electronics Engineering Dept., METU

\section{Examining Committee Members}

Prof. Dr. Erol Kocaoğlan

Electrical and Electronics Engineering Dept., METU

Prof. Dr. Mübeccel Demirekler

Electrical and Electronics Engineering Dept., METU

Prof. Dr. Kemal Leblebicioğlu

Electrical and Electronics Engineering Dept., METU

Prof. Dr.Tolga Çiloğlu

Electrical and Electronics Engineering Dept., METU

M.Sc.Uğur Kayasal

Roketsan Missiles Industries Inc.

Date:

$\underline{02.02 .2012}$ 
I hereby declare that all information in this document has been obtained and presented in accordance with academic rules and ethical conduct. I also declare that, as required by these rules and conduct, I have fully cited and referenced all material and results that are not original to this work.

Name, Last name : : Tamer AKÇA

Signature 


\begin{abstract}
AN ADAPTIVE UNSCENTED KALMAN FILTER FOR

TIGHTLY-COUPLED INS/GPS INTEGRATION
\end{abstract}

\begin{abstract}
Akça, Tamer
M. Sc., Department of Electrical and Electronics Engineering Supervisor: Prof. Dr. Mübeccel Demirekler
\end{abstract}

February 2012, 92 pages

In order to overcome the various disadvantages of standalone INS and GPS, these systems are integrated using nonlinear estimation techniques and benefits of the two complementary systems are obtained at the same time. The standard and most widely used estimation algorithm in the INS/GPS integrated systems is Extended Kalman Filter (EKF). Linearization step involved in the EKF algorithm can lead to second order errors in the mean and covariance of the state estimate. Another nonlinear estimator, Unscented Kalman Filter (UKF) approaches this problem by carefully selecting deterministic sigma points from the Gaussian distribution and propagating these points through the nonlinear function itself leading third order errors for any nonlinearity. Scaled Unscented Transformation (SUT) is one of the sigma point selection methods which gives the opportunity to adjust the spread of sigma points and control the higher order errors by some design parameters. Determination of these parameters is problem specific. In this thesis, effects of the SUT parameters on integrated navigation solution are investigated and an "Adaptive UKF" is designed for a tightly-coupled INS/GPS integrated system. Besides adapting process and 
measurement noises, SUT parameters are adaptively tuned. A realistic fighter flight trajectory is used to simulate IMU and GPS data within Monte Carlo analysis. Results of the proposed method are compared with standard EKF and UKF integration. It is observed that the adaptive scheme used in the sigma point selection improves the performance of the integrated navigation system especially at the end of GPS outage periods.

Keywords: INS/GPS; Adaptive Nonlinear Estimation; EKF; UKF; Unscented Transformation 


\title{
ÖZ
}

\author{
SIKI BAĞLI ANS/KKS TÜMLEŞTİRMESINDE \\ UYARLAMALI KOKUSUZ KALMAN FILTRESİ UYGULAMASI
}

\begin{abstract}
Akça, Tamer
Yüksek Lisans, Elektrik ve Elektronik Mühendisliği Bölümü

Tez Yöneticisi : Prof. Dr. Mübeccel Demirekler
\end{abstract}

Şubat 2012, 92 sayfa

Tek başlarına çalıştırıldıklarında çeşitli dezavantajları bulunan ataletsel navigasyon sistemi (ANS) ve küresel konumlama sistemi (KKS), doğrusal olmayan kestirim algoritmaları kullanılarak tümleştirilmekte ve birbirini bütünler nitelikte olan bu iki sistemin ayrı ayrı getirileri tek bir sistemden elde edilmektedir. Bu uygulama için en çok ve en yaygın bir biçimde kullanılan kestirim algoritması genişletilmiş kalman filtresidir (EKF). EKF uygulamasında yer alan doğrusallaştırma işlemleri nedeniyle kestirim sonucunun ortalama ve standart sapma değerlerinde ikinci dereceden hatalar oluşabilmektedir. Bu uygulamada kullanılabilecek bir başka doğrusal olmayan kestirim algoritması ise kokusuz kalman filtresidir (UKF). UKF, Gaussian dağılım içerisinden belirli bir şekilde seçilen örnekleme noktalarını, doğrusal olmayan sistem ve ölçüm modelinden geçirir ve ikinci dereceden hatalara sahip kestirim sonucunu elde eder. Bahsi geçen örnekleme noktalarının seçiminde kullanılan yöntemlerden biri Orantılanmış Kokusuz Dönüşümdür (SUT). Bu yöntem dahilindeki bazı değişkenler ile örnekleme noktalarının dağılımını belirleme ve yüksek dereceden kestirim hatalarının kontrolünü sağlama imkanı elde edilir. $\mathrm{Bu}$ değişkenlerin 
belirlenmesi ilgili probleme özgüdür. $\mathrm{Bu}$ tez kapsamında SUT değişkenlerinin tümleştirilmiş navigasyon sistemi üzerindeki etkileri değerlendirilmiş ve sıkı bağlı ANS/KKS tümleşik sistemi, "Uyarlamalı UKF" kullanılarak tasarlanmıştır. Bu uygulama kapsamında süreç ve ölçüm gürültülerinin dışında, SUT değişkenleri uyarlamalı olarak değiştirilmiştir. Gerçekçi bir savaş uçağı uçuş senaryosu ile, ataletsel ölçüm biriminin ve küresel konumlama sisteminin çoklu koşum analizi yöntemi dahilinde benzetimi yapılmıştır. Önerilen yöntem ile elde edilen sonuçlar, standart EKF ve UKF yöntemlerinin sonuçları ile kıyaslanmıştır. Sonuç olarak özellikle KKS sinyallerinin kesintiye uğradığı sürelerden sonra, önerilen yöntemin navigasyon sisteminin hassasiyetini arttırdığı gözlemlenmiştir.

Anahtar Kelimeler: ANS/KKS; Uyarlamalı doğrusal olmayan kestirim; EKF; UKF; kokusuz dönüşüm 
To My Family and My Endless Love Merve 


\section{ACKNOWLEDGEMENTS}

First of all, I would like to express my sincere thanks to my supervisor Prof. Dr. Mübeccel Demirekler for her complete guidance, advice, criticism and encouragement throughout the M.Sc. study.

I would like to express my appreciation to Roketsan Missile Industries Inc. for providing me a peaceful working environment and continuous support.

I also would like to thank my dear friend Recep Serdar Acar for his valuable ideas and encouragement throughout this study.

Finally, I would like to express my special thanks to my family for their permanent support and sincere love. 


\section{TABLE OF CONTENTS}

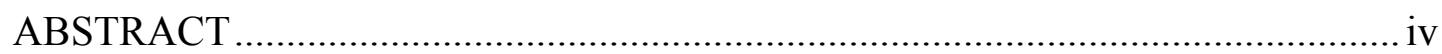

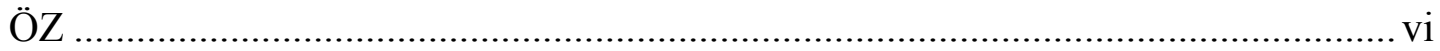

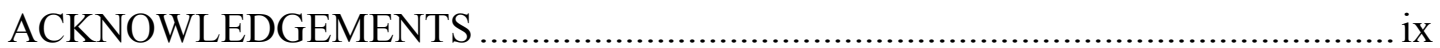

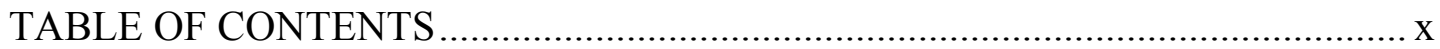

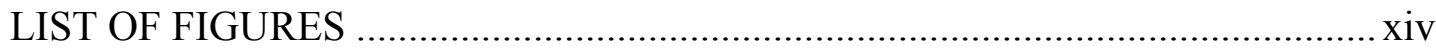

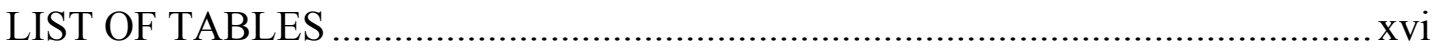

CHAPTERS

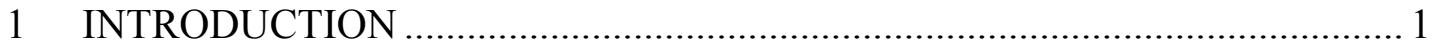

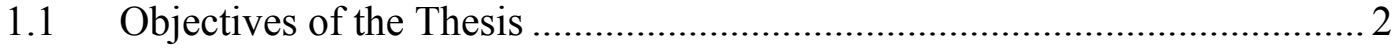

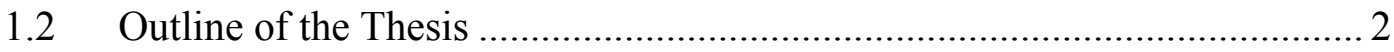

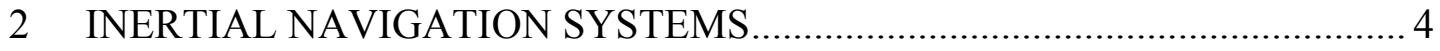

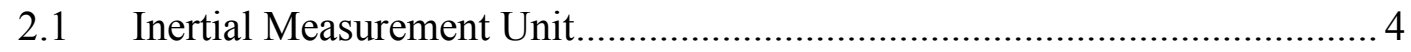

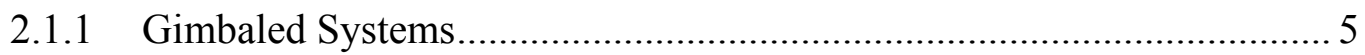

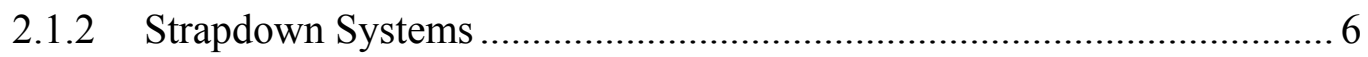

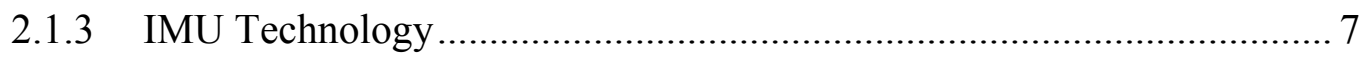

2.1.3.1 Accelerometer Technology ......................................................... 7

2.1.3.1.1 Force Feedback Accelerometer ................................................ 7

2.1.3.1.2 Vibrating Beam Accelerometer ............................................ 8

2.1.3.1.3 MEMS Accelerometer......................................................... 9

2.1.3.2 Gyroscope Technology ........................................................... 10

2.1.3.2.1 Spinning Mass Gyroscope ...................................................... 10

2.1.3.2.2 Ring Laser Gyroscope ................................................................ 11 
2.1.3.2.3 Fiber Optical Gyroscope...................................................... 12

2.1.3.2.4 Coriolis Gyroscope ........................................................... 13

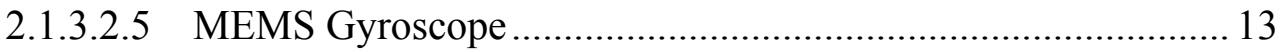

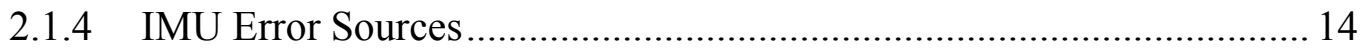

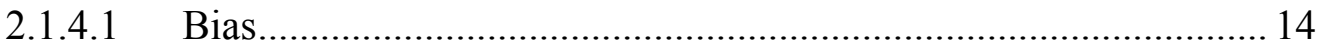

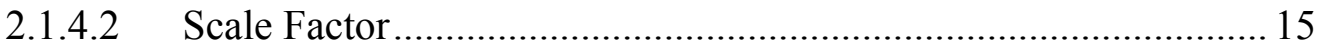

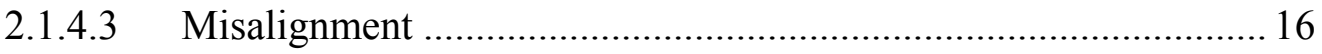

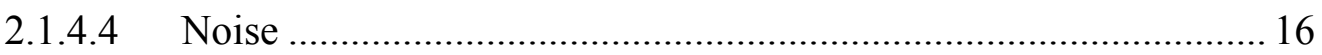

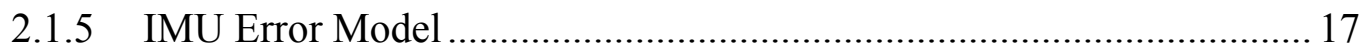

2.2 Inertial Navigation System (INS) Dynamics......................................... 18

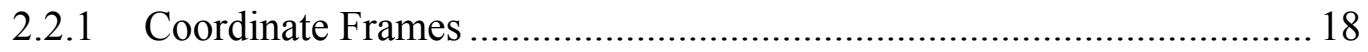

2.2.1.1 Inertial Frame (I-Frame) …...................................................... 18

2.2.1.2 Earth Frame (E-Frame) ….......................................................... 18

2.2.1.3 Navigation Frame (N-Frame) ….............................................. 19

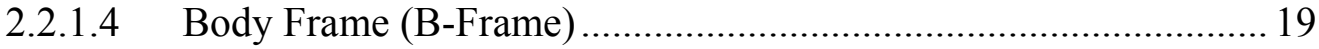

2.2.2 Earth Model....................................................................................... 19

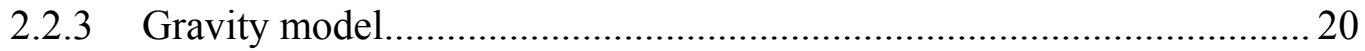

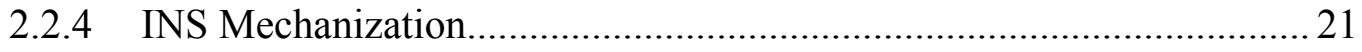

2.2.4.1 Attitude Mechanization........................................................... 22

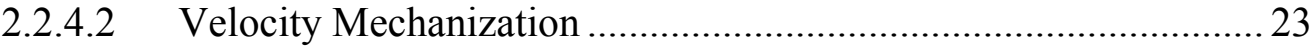

2.2.4.3 Position Mechanization............................................................... 24

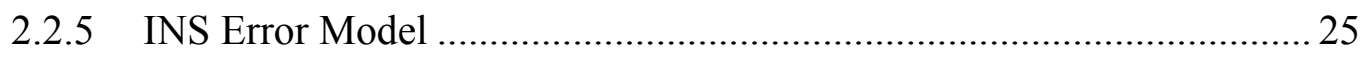

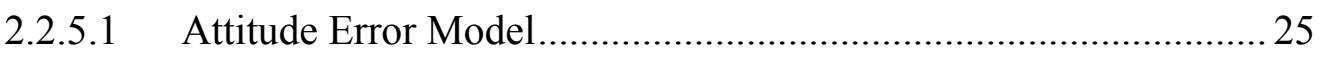

2.2.5.2 Velocity Error Model ........................................................... 27

2.2.5.3 Position Error Model......................................................................... 27

2.2.5.4 State Space Error Model …........................................................ 28 


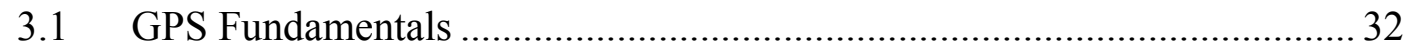

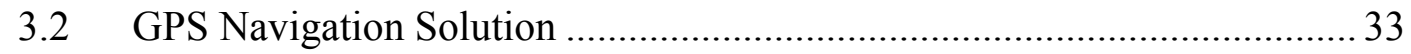

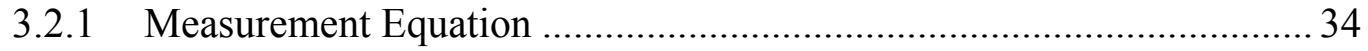

3.2.2 Least Squares Estimate of The Navigation Solution............................. 35

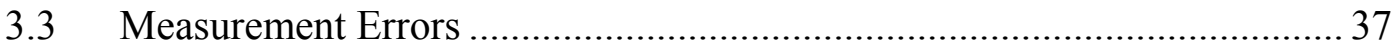

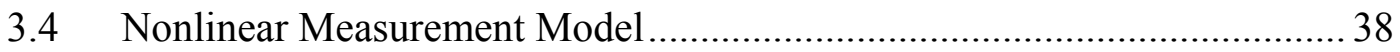

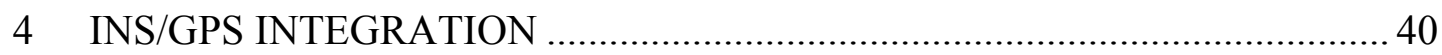

4.1 Benefits and Drawbacks of Each System ............................................... 40

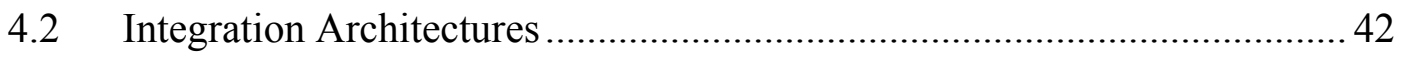

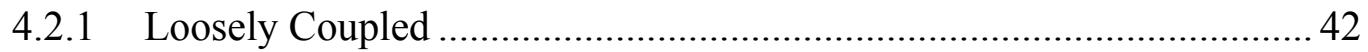

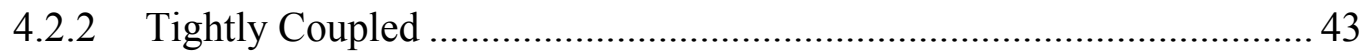

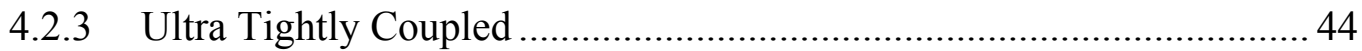

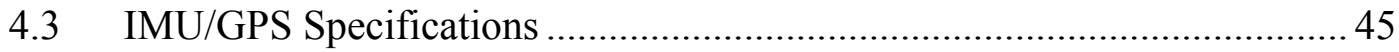

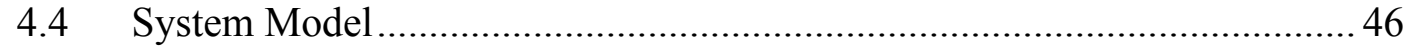

4.5 Discrete Time Equivalent System Model.............................................. 47

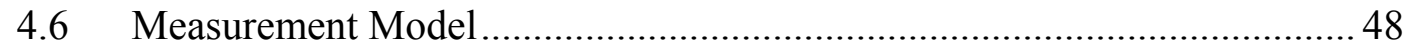

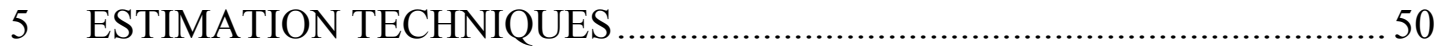

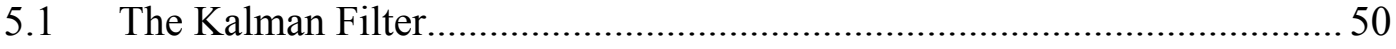

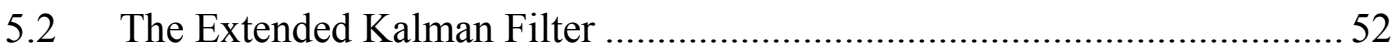

$5.3 \quad$ The Unscented Kalman Filter................................................................. 54

$5.4 \quad$ The Adaptive Unscented Kalman Filter ..................................................5

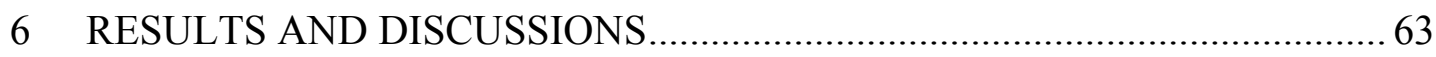

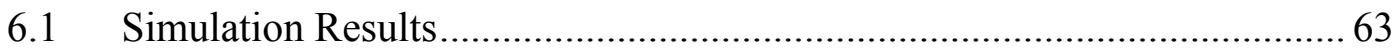

6.1.1 Reference and Standalone Inertial Navigation Results .......................63

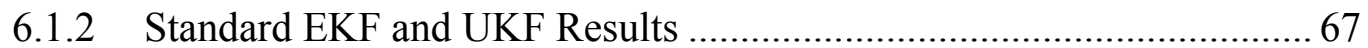




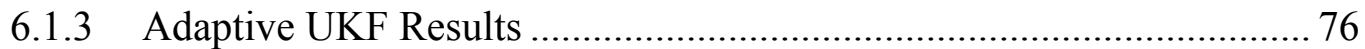

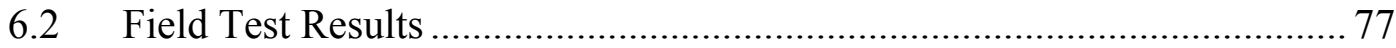

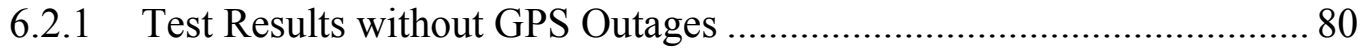

6.2.2 Test Results with GPS Outages .................................................... 83

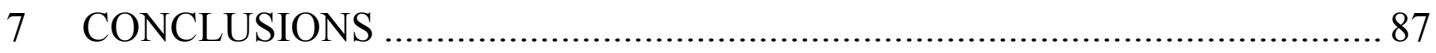




\section{LIST OF FIGURES}

\section{FIGURES}

Figure 1 Inertial measurement units (Figure is adapted from [8]) ............................ 6

Figure 2 Schematic of a force feedback accelerometer (Figure is adapted from [9]).. 8

Figure 3 Schematic of a Vibrating beam accelerometer (Figure is adapted from [9]) 9

Figure 4 Basic components of laser gyroscopes (Figure is adapted from [8]).......... 12

Figure 5 Schematic of the INS mechanization equations ...................................... 22

Figure 6 Flowchart of loosely coupled architecture ................................................. 43

Figure 7 Flowchart of tightly coupled architecture................................................. 44

Figure 8 Flowchart of ultra tightly coupled integration ........................................ 44

Figure 9 RMSE variations over alpha values for Steady state conditions ................. 61

Figure 10 RMSE variations over alpha values for 20 seconds GPS Outage ............. 61

Figure 11 RMSE variations over alpha values for 30 seconds GPS Outage .............62 62

Figure 12 RMSE variations over alpha values for 40 seconds GPS Outage ............. 62

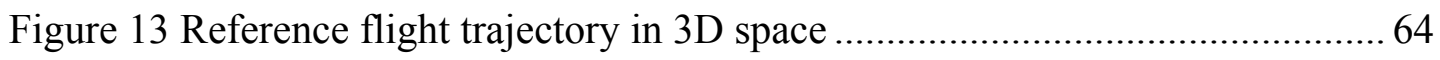

Figure 14 Standalone INS and reference flight trajectories in 3D space ..................6 64

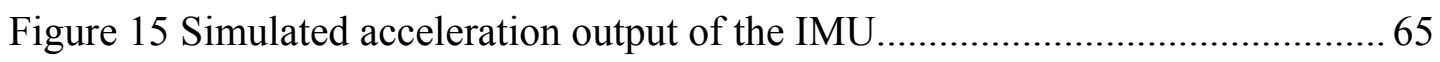

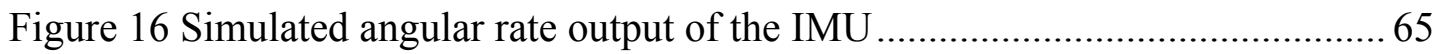

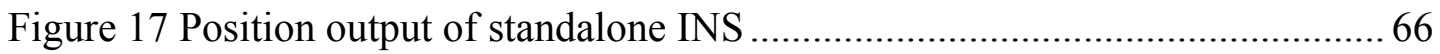

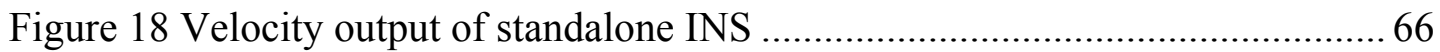

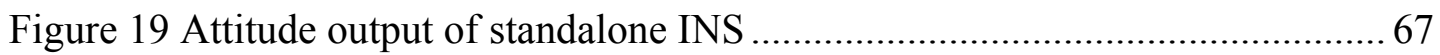

Figure 20 EKF Position estimation error and $\pm 1 \sigma$ error bound.................................6 69

Figure 21 EKF Velocity estimation error and $\pm 1 \sigma$ error bound ...............................6 69

Figure 22 EKF Attitude estimation error and $\pm 1 \sigma$ error bound............................... 70

Figure 23 EKF Accelerometer bias estimation error and $\pm 1 \sigma$ error bound............... 70

Figure 24 EKF Accelerometer bias estimation error and $\pm 1 \sigma$ error bound................ 71 
Figure 25 UKF Position estimation error and $\pm 1 \sigma$ error bound .............................. 71

Figure 26 UKF Velocity estimation error and $\pm 1 \sigma$ error bound .............................. 72

Figure 27 UKF Attitude estimation error and $\pm 1 \sigma$ error bound ............................. 72

Figure 28 UKF Accelerometer bias estimation error and $\pm 1 \sigma$ error bound .............. 73

Figure 29 UKF Gyroscope bias estimation error and $\pm 1 \sigma$ error bound...................... 73

Figure 30 Comparison of EKF and UKF RMSE metrics ..................................... 74

Figure 31 Position RMSE values of EKF\&UKF (Zoomed version of Figure 30)..... 75

Figure 32 Navigation Systems: a. FOG INS/GPS- CNS5000, b. MEMS IMU-MTi 77

Figure 33 Test set-up for the reference system (Figure is adapted from [38]) .......... 78

Figure 34 Acceleration measurements of CNS5000 (REF) and MTi (MEMS) ........ 79

Figure 35 Angular rate measurements of CNS5000 (REF) and MTi (MEMS)......... 79

Figure 36 3D Flight trajectory computed by the reference system and AUKF ......... 80

Figure 37 Horizontal position computed by the reference system and AUKF ......... 81

Figure 38 Position output computed by the reference system and AUKF................ 81

Figure 39 Velocity output computed by the reference system and AUKF ................ 82

Figure 40 Attitude output computed by the reference system and AUKF................ 82

Figure 41 AUKF Position estimation error and $\pm 1 \sigma$ error bound ............................ 84

Figure 42 AUKF Position estimation error and $\pm 1 \sigma$ error bound ............................ 84

Figure 43 AUKF Position estimation error and $\pm 1 \sigma$ error bound …........................ 85

Figure 44 AUKF Position estimation error and $\pm 1 \sigma$ error bound ............................ 85 


\section{LIST OF TABLES}

\section{TABLES}

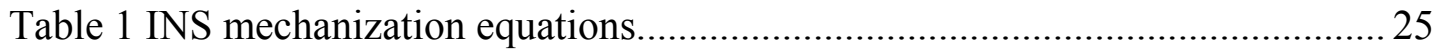

Table 2 Benefits and drawbacks of INS and GPS............................................... 41

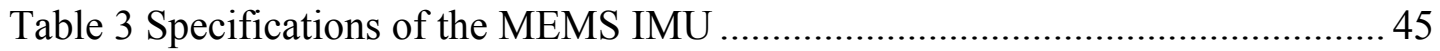

Table 4 Specifications of the GPS receiver observables........................................... 46

Table 5 The Kalman Filter Algorithm .................................................................. 51

Table 6 The Extended Kalman Filter Algorithm ........................................................ 53

Table 7 Scaled Unscented Transformation Algorithm............................................ 55

Table 8 The Unscented Kalman Filter Algorithm [32] ........................................... 56

Table 9 Spread of $\sigma$-points for different $\alpha$ parameters .........................................58

Table 10 Optimal Alpha values and RMSE improvement percentage .....................59

Table 11 Generalized variance intervals for different GPS outage periods ............... 60

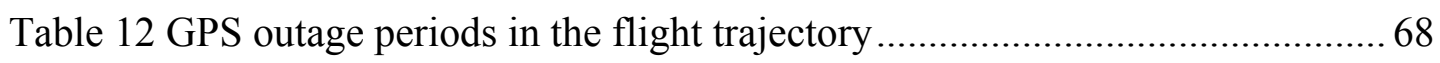

Table 13 RMSE Performance comparison of EKF and UKF …............................. 75

Table 14 RMSE Performance comparison of EKF, UKF and AUKF ...................... 76

Table 15 Field test RMSE performance comparison of EKF, UKF and AUKF....... 83

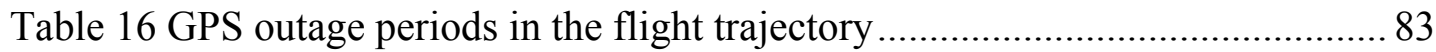

Table 17 Field test RMSE performance comparison of EKF, UKF and AUKF........86 


\section{CHAPTER 1}

\section{INTRODUCTION}

Inertial Navigation Systems (INS) are designed in order to determine the velocity, position and the attitude of a moving object. These systems are originally developed for navigating rockets during the Second World War and widely used in marine, aerospace and land navigation areas [1]. Such a system is mainly composed of accelerometers, gyroscopes and computers which make use of the outputs of these sensors. Both, accelerometers and gyroscopes operate on the inertial principles (Newton's Laws of Motion) while forming linear acceleration and angular velocity measurements. For this reason, the system composed of these inertial sensors is named as Inertial Navigation System [2]. The sensor cluster formed by three accelerometers and three gyroscopes is called as inertial measurement unit which is the most significant component of INS.

Global Positioning System (GPS) is a satellite based radio navigation system that provides three dimensional navigation solution. GPS project was developed in 1973 to improve the performance of the previous navigation systems. The system became fully operational in 1994 and made freely available for the civilian use [3]. The basic operation of the GPS is obtaining user position and velocity using the radio signals broadcast by the satellites. Navigation solution is basically obtained by comparing the transmission and receiving times of the GPS signals.

Both INS and GPS suffer from various error sources and deficiencies which propel the accompaniment of the two complementary systems. Inertial navigation systems 
exhibit relatively low noisy outputs which tend to drift over time [4]. Contrary to INS, GPS outputs are relatively noisy but do not exhibit long-term drift [4]. Using both of these systems together results in a superior navigation performance than either alone. Integrating the outputs from each sensor results in a system which can be viewed as a drift free INS [5].

\subsection{Objectives of the Thesis}

In the process of INS/GPS integration, various estimation techniques have been utilized. The most widely used algorithm is the Extended Kalman Filter which is based on linearized system and measurement models [6]. In order to improve the performance of the integration, different methods are proposed and implemented. In the scope of this thesis, an Adaptive Unscented Kalman Filter implementation is used to overcome the deficiencies of the Extended Kalman Filter and improve the navigation performance of the INS/GPS integrated system.

\subsection{Outline of the Thesis}

Chapter 2 presents the fundamental information about the inertial navigation systems. The Earth and the gravity models, error model of the inertial measurement unit, the INS mechanization equations, and the linear error model of the INS are all included in this chapter.

Chapter 3 provides the fundamental characteristics of the Global Positioning System. After the background information, methods for obtaining the GPS navigation solution are investigated. The definitions of the sources of measurement errors are discussed and a nonlinear measurement model is obtained at the end of the chapter.

Chapter 4 discusses the benefits and drawbacks of the INS and the GPS in detail by considering their performance, cost and functionality. INS/GPS integration architectures, loosely coupled, tightly coupled and deeply coupled, are defined. 
Specifications of the utilized sensor systems are supplied. Finally, the state space model and the measurement model for the INS/GPS integration process are constituted.

Chapter 5 provides the background information about the nonlinear estimation algorithms, Extended Kalman Filter and Unscented Kalman Filter. The adaptive scheme utilized for the Unscented Kalman Filter is discussed. Pseudo codes for each algorithm are supplied in related sections.

Chapter 6 presents overall results of the simulations, tests and discussions about the utilized methods.

Chapter 7 provides a brief summary and conclusions of the thesis study. The comparison of the results and the future work of the thesis are mentioned. 


\section{CHAPTER 2}

\section{INERTIAL NAVIGATION SYSTEMS}

This chapter provides the background information about the inertial navigation systems, which is necessary in order to understand the nature and principles of operation of the inertial navigation. Equations serving as the framework for INS/GPS integration are explained and clarified starting from the sensor level then proceeding to the system level. After making a brief introduction to inertial measurement units, basic design and production technologies for inertial sensors are considered. Then IMU error model is obtained using the predefined inertial sensor error source models. Remaining part of the chapter is devoted to the INS dynamics which includes the definitions of reference frames, the Earth and gravity models, INS mechanization equations, INS error model and state space error model for the INS/GPS integration process in turn.

\subsection{Inertial Measurement Unit}

An inertial measurement unit is composed of three gyroscopes and three accelerometers which are orthogonally mounted on a fixture. These sensors are mainly used to determine the current state of the system in three dimensional space. Also flight control systems and stabilized platforms make use of the IMU outputs [7].

Angular velocity of the system is measured by gyroscopes. Via integrating angular velocities in three axes, angular position of the system can be obtained considering 
the initial conditions. Angular velocities can be regarded as the rate at which the system rotates around a given axis.

Linear acceleration of the system is measured by accelerometers. Once integrating the linear acceleration, linear speed is obtained. By integrating the speed, change in the position can be found. With the use of initial conditions, current velocity and position of the system can be obtained.

Literally, large number of designs exists for inertial measurement units. These units are classified according to their sensor technologies and areas of interests. In general, these systems are categorized into two groups, namely gimbaled systems and strapdown systems.

\subsubsection{Gimbaled Systems}

The sensor cluster consisting of three gyroscopes and three accelerometers is rigidly mounted to the gimbal part in gimbaled systems. In this setup, sensors are isolated from the rotations of the outer system. Attitude of the sensor cluster does not change even the vehicle rotates. The sensor cluster is stabilized by using feedback from the gyroscopes which sense the rotation of the outer system. By using sensors with precise measurement, very accurate results can be obtained from this system. One drawback of this system is the gimbal lock phenomena. When two of the gimbals align themselves parallel to each other, rotations of the sensor cluster around one of the three axes can not be eliminated. A fourth gimbal is required to overcome this problem [8].

Gimbals are very expensive and sophisticated devices. High cost can be considered as a disadvantage of this system. The advantage is that, many sensor errors are eliminated and more accurate results are obtained by stabilizing the inertial sensors. 


\subsubsection{Strapdown Systems}

The sensor cluster is rigidly mounted to the axis of the moving object in strapdown systems. Therefore inertial sensors are not stabilized, in other words they follow the motion of the outer vehicle. They experience higher rotation rates than the gimbaled systems. Due to the higher rotation rates, outputs of the sensors become more erroneous and more complicated error correction mechanisms are needed to compensate for the deviations from the exact data.

Strapdown systems can be preferred in wide range of applications as they have smaller size and lower weight. Most importantly, they reduce the cost, power consumption and complexity of the system drastically. As a result, with the accompaniment of the improvements in computation power of onboard computers since 70's, strapdown systems became the most common configuration [8]. In this thesis work, a strapdown inertial navigation system is modeled. Diagram of the internal structure of two systems can be seen in the figure below.

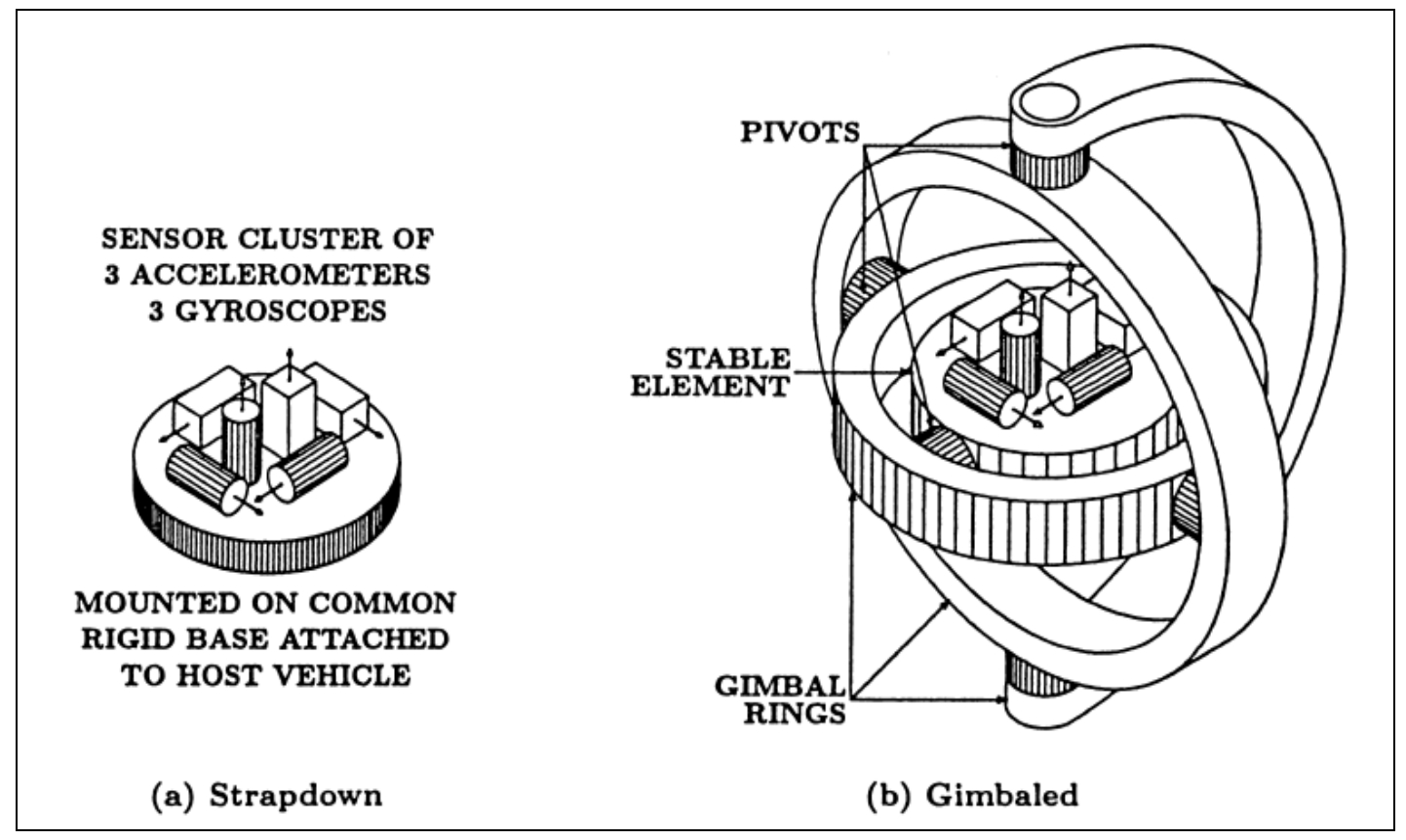

Figure 1 Inertial measurement units (Figure is adapted from [8]) 


\subsubsection{IMU Technology}

Basic operational principles and technological basis of accelerometers and gyroscopes are discussed in this section.

\subsubsection{Accelerometer Technology}

Most of the accelerometers are based on force feedback or vibrating beam technology. Both technologies share the same principle of measuring the force acting on a proof mass instead of the vehicle [9].

\subsection{Force Feedback Accelerometer}

These types of accelerometers are also called as "pendulous mass". A proof mass is connected to the accelerometer case by the help of a pendulous arm. The proof mass is made free to move only in the input axis direction by supporting it with a hinge in two dimensions. Acceleration of the vehicle causes a force to act on the proof mass which in turn results in a deflection in the position of the proof mass. An external force which is proportional to the acceleration is required to stabilize the proof mass. The deflection of the proof mass is detected by a pick-off system. The signal generated by this system is used in a torquer system in order to move the proof mass back to its null position. The force applied by the torquer system is proportional to the acceleration of the host system [10]. A simple schematic of a force feedback accelerometer can be seen in Figure 2 


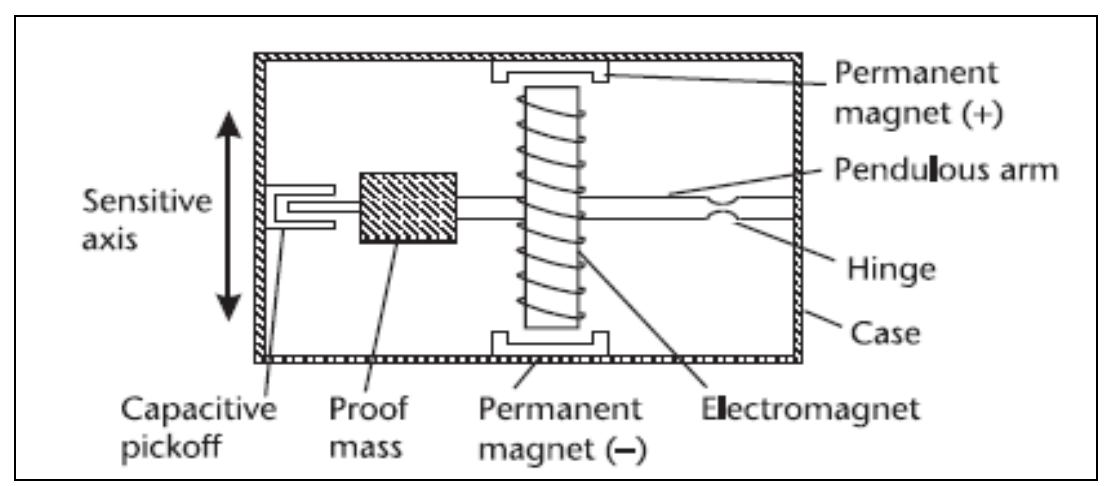

Figure 2 Schematic of a force feedback accelerometer (Figure is adapted from [9])

Qualities of the components (hinge, pendulous arm, proof mass, torquer, pick-off system...) are main factors affecting the performance of sensors. Different grades of performance can be obtained at different prices by varying the component quality. Most of the high performance accelerometers are mechanical force feedback type of technology [9].

\subsection{Vibrating Beam Accelerometer}

These types of sensors are also called as "resonant accelerometers". Vibrating Beam accelerometer uses the same proof mass and pendulous arm structure as force feedback accelerometers. But the proof mass is supported along the sensitive axis by a vibrating beam at its resonant frequency. When there is acceleration along the sensitive axis, the proof mass deflects and compresses or tenses the vibrating beam which constrains its motion. The resonant frequency of the beam is decreased by compression and increased by the tension caused by proof mass. As a result, acceleration of the system can be obtained by measuring the resonant frequency of the vibrating beam [11]. 


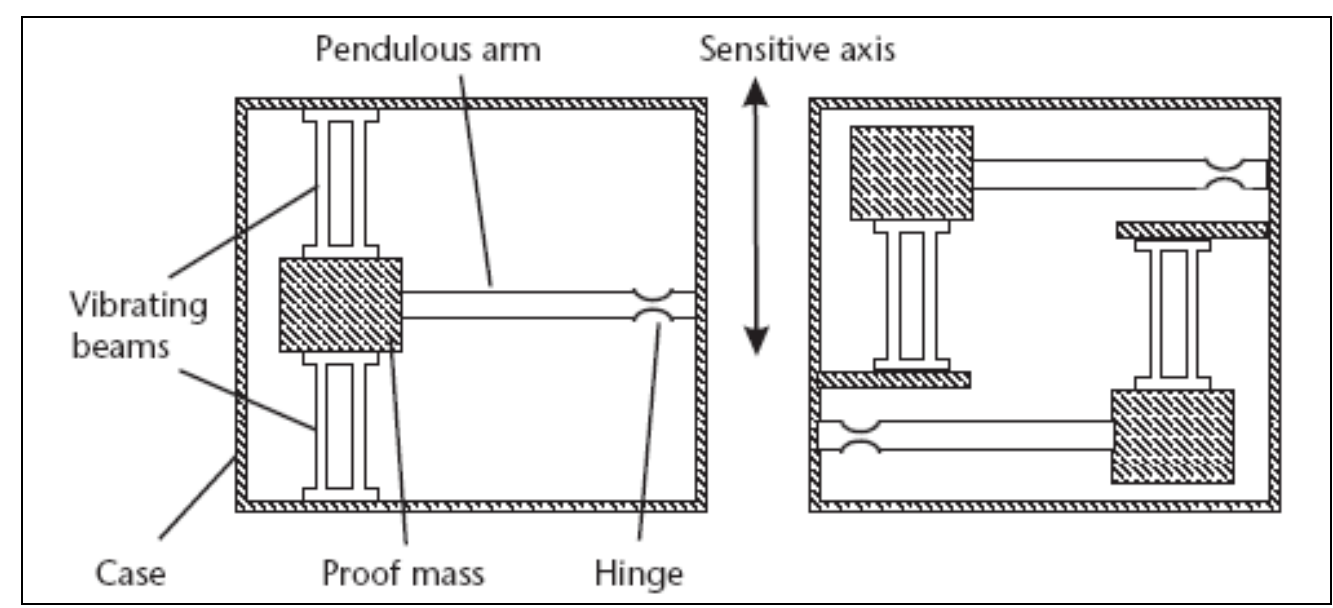

Figure 3 Schematic of a Vibrating beam accelerometer (Figure is adapted from [9])

\subsection{MEMS Accelerometer}

Development of the low cost micro sensors and micro actuators became realizable with the arrival of MEMS technology. This technology enables locating complete systems on a single chip. Inertial sensors are typical modern applications of this technology. With the features of low cost, low power consumption and small size, these devices overcome most of the design requirements. MEMS accelerometers are quickly replacing conventional accelerometers. They are being developed and implemented in a wide range of commercial applications like automotive industry. Actually, MEMS accelerometers do not represent another measurement principle. It uses the same principles of operations but utilizes another production technique. Both pendulous-mass and vibrating beam types of accelerometers can be built by MEMS technology [10].

By using silicon or quartz, very small mechanical structures are produced enabling three axis accelerometers and their electronics to be integrated in a common chip. However, as the size of the sensing instruments decreases, measurement performance and sensitivity decrease meaning a higher instrument error and noise level. In the case of low measurement quality, MEMS inertial sensors can not be used as a standalone navigation system [12]. Deterministic errors of the sensors should be compensated and random errors should be accurately estimated throughout the usage period in order to obtain an acceptable performance. 
In the scope of this study, MEMS accelerometers are selected as the linear acceleration measurement source and modeled accordingly.

\subsubsection{Gyroscope Technology}

Gyroscope technology is more complicated and more diverse than accelerometers. There have been many different solutions for the design and production of gyroscopes. Gyroscope technology can be discussed in four main types namely spinning mass, optical, coriolis and MEMS gyroscopes [10]. Also there are two types of optical gyroscopes, that is ring laser and fiber optic gyroscopes, using the same principle of operation but different technological basis. All of these technologies are handled separately in the following parts.

\subsection{Spinning Mass Gyroscope}

Gyroscopic theory is the fundamental method of measuring rotation of a body without using an external reference [10]. When a spinning mass is rotated around its input axis perpendicular to its spin axis, a reaction will take place in the third axis which is perpendicular to both. The amount of this reaction is an indicator for the rate of input rotation. This principle forms the basis for all mechanical gyroscopes [10].

There exist different types of spinning mass gyroscopes such as dynamically tuned gyroscope, electrostatically suspended gyroscope, magnetically suspended gyroscope etc. A dynamically tuned gyroscope eliminates the movement in the reaction axis by using an electrical rebalance loop. The input rotation rate is proportional to the amount of required current for balancing the spinning mass. Different gyroscope performances can be obtained at different prices depending on the quality of control electronics, spin motor and torquers [9]. 


\subsection{Ring Laser Gyroscope}

RLG consists of a triangular laser cavity with mirrors located at the vertices of closed loop with at least three arms. A beam of laser is split into two beams, one of which travels in the clockwise direction and the other in the counter-clockwise direction. Mirrors at the vertices are used to form a continuous light path. Laser beams generated travel around this path being reflected from each mirror, and return to its initial starting point. In case of the sensor being stationary, both beams have the same frequency. When the gyro rotates at some angular rate, optical path lengths change, i.e., the distance traveled by the beam travelling in the opposite direction of the rotation becomes shorter than the other one. The frequency of each beam changes to maintain the resonant condition. This results in a phase difference between two travelling waves. This phenomenon is called "Sagnac Effect". The phase shift can be measured by an interferometer. This output is proportional to the rate of rotation [11].

The drawback of this technology is the laser lock phenomena. At low rotation rates, the phase difference measured can be equal to zero as the result of back scattering. This causes the beams to synchronize and travel at the same rates in the lock-in state. At this state, the device will not accurately track its angular position over time meaning that no measurements are taken. One of the methods to lessen this problem is using mechanical oscillation. Angular vibrations are applied to the whole laser cavity at high frequency and low amplitude through small angles. Drawback of this method is an increase in size, weight and complexity of the system. The output should be compensated optically or electronically for the oscillatory motion. There are no moving parts in RLG structure meaning that there is no friction. Therefore there will not be inherent drift terms, which is an advantage of the system. Furthermore the entire unit is compact, light weighted and virtually indestructible. The primary disadvantage of RLG technology is the requirement for polishing the laser blocks and difficulties in producing the mirrors. High technology methods must be used to produce the mirrors that increase the cost of the device. RLG technology is still advancing, and it is at the practical limits of this technology [13]. 


\subsection{Fiber Optical Gyroscope}

Its principle operation depends on measuring the "Sagnac Effect" as in the case of RLG. An external laser source exists in the FOG which generates travelling beams both in the clockwise and in the counter-clockwise directions. In this setup, the light waves travel in a fiber-optic cable. When the device experiences inertial rotation, the distances travelled by two counter waves become different due to the sagnac effect. This operation results in two different frequencies for two beams which mean a phase difference between the laser beams. The output phase difference measured is proportional to the rotation rate of the device [11].

Meanwhile, temperature changes and accelerations may disrupt the structure of the optical fiber. This is one of the sources of error which should be minimized by using design techniques. On the other hand fiber optic gyroscopes do not include mirrors or gas in their internal design. Furthermore, lock-in phenomena is not inherent in FOG technology unlike the RLG. These properties make the FOG technology cheaper compared to RLG. In these days FOGs are replacing RLGs in the lower performance tactical and commercial applications. Improvements in the FOG performance will make it possible to use these devices in strategic applications with performance requirements of $0,001 \mathrm{deg} / \mathrm{h}$ [10]. In the implementation of FOG; fiber-optic sensing coil, a light source and a photo detector is used. In the figure below, internal structures of two types of laser gyros (RLG, FOG) can be seen.

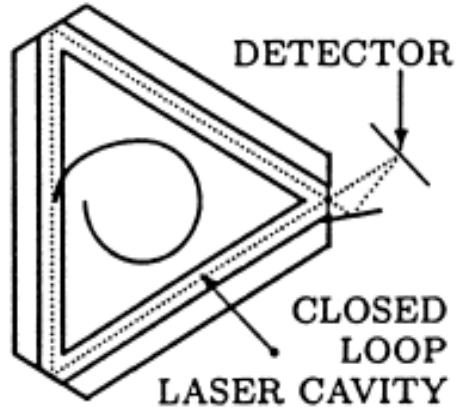

(a) Ring Laser Gyro (RLG)

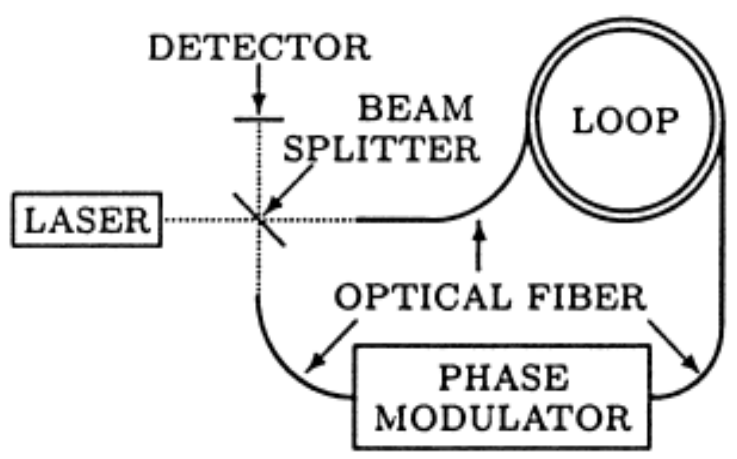

(b) Fiber Optic Gryo (FOG)

Figure 4 Basic components of laser gyroscopes (Figure is adapted from [8]) 
By the help of developments in the FOG technology, Interferromagnetic Fiber Optical gyroscope (IFOG) is invented. This device has brought substantial increases in the performance of gyroscopes. This technology has been used in many lower grade applications as unmanned vehicles, stabilization systems etc. [8]. Measurement accuracy of IFOGs is approaching to RLGs but due to high prices, it is not used widely. However with increasing industrial investments, the costs will decrease and IFOG is expected to replace RLG in high performance applications. With the implementation of IFOG technology, integrated guidance and navigation systems have the potential of having very low cost with high reliability and suitability for a wide range of military and commercial applications [13].

\subsection{Coriolis Gyroscope}

Coriolis gyroscopes are also called as vibratory gyroscopes. In this type of sensors, there exists an element which undergoes a simple harmonic motion in a plane. The shapes of this vibrating element may be tuning fork, ring, hemisphere, cylinder, spring, beam or pair of beams [9]. All types of these gyroscopes have the same principle of operation, which can be summarized as detecting the coriolis acceleration of the vibrating element when it is subject to a rotation. A sinusoidal vibration is induced in a plane perpendicular to the vibration of the element when the gyroscope is rotated. The amount of the induced vibration is proportional to the rotation rate. Most of the coriolis gyroscopes have low performance specifications with a low price. Hemispherical resonator gyroscope is an exception that it can indeed offer high performance [10].

\subsection{MEMS Gyroscope}

As stated in section 2.1.3.1.3 about MEMS accelerometers, MEMS gyroscopes do not represent a different measurement principle. They use the same principles of operations but utilize another production technique. MEMS gyroscopes are generally based on coriolis principles discussed in section 2.1.3.2.4. These gyroscopes are 
produced in very small sizes and micro-machined directly on silicon or similar substrates. It is noted that better performances can be obtained by using quartz rather than silicon substrates [9]. Basic advantages of this technology are its mass production capability, very low cost with high quantity, very small size, minimal power requirements and resistance to very high shock. In the context of this thesis, a MEMS gyroscope with moderate performance is modeled.

\subsubsection{IMU Error Sources}

The outputs of the inertial sensors exhibit definite types of errors to some extent depending on their performance. Bias, scale factor, misalignment and noise are the basic systematic error sources of accelerometers and gyroscopes. Each of these error sources has four different characteristics namely, a deterministic fixed component, a temperature dependent component, a turn-on to turn-on component and an in-run variation component [9]. First two deterministic components of sensor errors can be compensated on the IMU processor by using the IMU calibration data. The procedures for obtaining the calibration database for inertial sensors are clearly defined by IEEE standards [14]. The remaining two other error components have a stochastic behavior which determines the performance of the IMU. In the context of this study, it is assumed that the deterministic errors of the IMU outputs are compensated perfectly. Since post calibration performance of the IMU is major in determining the inertial navigation performance, only stochastic errors are modeled while forming the inertial data [9]. Stochastic error characteristics of accelerometers and gyroscopes are discussed in detail.

\subsubsection{Bias}

Inertial sensor bias is defined as the constant error present in the average of the sensor output over a specified time measured at specified operating conditions [14]. Bias error is independent of the underlying acceleration or angular rate of the sensor. It is convenient to consider bias in two components as static and dynamic biases. 
Static bias is also known as turn-on to turn-on bias or bias repeatability. This component of the bias is set at the power up and it is constant throughout IMU operation period. But it varies from run to run. Bias repeatability also includes residual fixed bias remaining after the calibration of sensor [9]. The variation of the bias repeatability can be represented by a Gaussian distribution and this component can be modeled as a random constant as follows.

$$
\begin{aligned}
& \delta \dot{a}_{\text {bias }}=0+v_{\text {accbias }} \\
& \delta \dot{\omega}_{\text {bias }}=0+v_{\text {gyrobias }}
\end{aligned}
$$

Where $v_{\text {accbias }}, v_{\text {gyrobias }} \sim$ zero mean gaussian white noise

Dynamic bias is also known as in-run bias variation or bias stability. This component of the bias varies over periods of order of a minute depending on the characteristics of sensors. Bias stability also includes temperature dependent bias remaining after the calibration of sensor. Typical value of the bias stability is about 10 percent of bias repeatability [9]. Bias stability can be modeled as a first order Gaussian Markov process.

\subsubsection{Scale Factor}

Scale factor is a parameter that is used to obtain acceleration or angular rate values from the voltage outputs of accelerometers and gyroscopes. Scale factor is defined as the departure of slope of the input-output curve of an inertial sensor from unity after the unit conversion by IMU [14]. The scale factor errors of the accelerometers and gyroscopes are proportional to acceleration and angular rates along the sensitive axis respectively. Similar to bias, it is convenient to separate scale factor as static and dynamic components.

Static component of the scale factor, namely scale factor repeatability is set at the power up of the instrument and remains constant throughout IMU operation period. It also includes the residual error left after the calibration of sensor. Scale factor repeatability can be modeled as a random constant [9]. 
Dynamic component of the scale factor, namely scale factor stability is the dual of bias stability. This component includes temperature dependent scale factor error remaining after the calibration of sensor and represents the variation of the scale factor error over the operating periods. Scale factor stability can also be modeled as a first order Gaussian Markov process [9].

\subsubsection{Misalignment}

Because of the manufacturing limitations, sensitive axis of the inertial sensors cannot be placed orthogonally. This makes each accelerometer and gyroscope sensitive to the accelerations and angular rates along other axis which results in a cross-coupling error. Scale factor and misalignment errors are unitless quantities which are typically expressed in parts per million (ppm). Misalignment errors can be modeled as random constants [7].

\subsubsection{Noise}

Random noise is an additional error resulting from the internal structure of inertial sensors and sensor electronics. In general, random noise is a non-systematic, stochastic process which cannot be compensated by using deterministic models. Random noise of the inertial sensors can be modeled as zero mean additive white noise. Random noise of the accelerometers and gyroscopes is generally described as velocity random walk and angular random walk respectively. Noise on the accelerometer measurements is integrated to obtain velocity random walk. Similarly, noise on the gyroscope measurements is integrated to obtain angular random walk. Magnitudes of the random walk process are proportional to the square root of the integration time [9]. 


\subsubsection{IMU Error Model}

Accelerometers and gyroscopes exhibit further error characteristics such as bias instability, g-dependent bias, g-square dependent bias, scale factor nonlinearity, quantization noise etc. [9]. But these errors have negligible effect on the system performance for a short period of operation time in the order of a few minutes and they are out of scope of this study. The major error sources which have significant contribution to the growth of errors in inertial navigation systems are bias repeatability, scale factor repeatability, misalignment and random noise [4]. In the simulations, first three parameters are set randomly at the beginning of each run and held constant through that individual run. Random noise is generated at each time step by randomly sampling from a zero mean Gaussian distribution whose standard deviation is determined by the sensor specifications. Bias and scale factor stability errors are not modeled since the simulation time is comparable with the correlation time of these errors. The mathematical models, constituted in order to represent realistic IMU errors by injecting predefined errors to the reference accelerometer and gyroscope outputs are given in equations (2.2) and (2.3) respectively.

$$
\left(\begin{array}{c}
\tilde{f}_{x}^{b} \\
\tilde{f}_{y}^{b} \\
\tilde{f}_{z}^{b}
\end{array}\right)=\left(\left(\begin{array}{l}
b_{x}^{a c c} \\
b_{y}^{a c c} \\
b_{z}^{a c c}
\end{array}\right)+\left(\begin{array}{ccc}
1 & M_{x y}^{a c c} & M_{x z}^{a c c} \\
M_{y x}^{a c c} & 1 & M_{y z}^{a c c} \\
M_{z x}^{a c c} & M_{z y}^{a c c} & 1
\end{array}\right)\left(\begin{array}{c}
f_{x}^{b} \\
f_{y}^{b} \\
f_{z}^{b}
\end{array}\right)\right)\left(\begin{array}{ccc}
1+S_{x}^{a c c} & 0 & 0 \\
0 & 1+S_{y}^{a c c} & 0 \\
0 & 0 & 1+S_{z}^{a c c}
\end{array}\right)+\left(\begin{array}{l}
w_{x}^{a c c} \\
w_{y}^{a c c} \\
w_{z}^{a c c}
\end{array}\right)
$$

Where $f^{b}, \tilde{f}^{b}, b^{a c c}, M^{a c c}, S^{a c c}, w^{a c c}$ stand for reference acceleration, accelerometer sensed acceleration, bias, misalignment, scale factor and noise parameters for modeled accelerometer respectively.

$$
\left(\begin{array}{c}
\tilde{\omega}_{x}^{b} \\
\tilde{\omega}_{y}^{b} \\
\tilde{\omega}_{z}^{b}
\end{array}\right)=\left(\left(\begin{array}{l}
b_{x}^{\text {gyro }} \\
b_{y}^{\text {gyro }} \\
b_{z}^{\text {gyro }}
\end{array}\right)+\left(\begin{array}{ccc}
1 & M_{x y}^{\text {gyro }} & M_{x z}^{\text {gyro }} \\
M_{y x}^{\text {gyro }} & 1 & M_{y z}^{\text {gyro }} \\
M_{z x}^{\text {gyro }} & M_{z y}^{\text {gyro }} & 1
\end{array}\right)\left(\begin{array}{c}
\omega_{x}^{b} \\
\omega_{y}^{b} \\
\omega_{z}^{b}
\end{array}\right)\right)\left(\begin{array}{ccc}
1+S_{x}^{\text {gyro }} & 0 & 0 \\
0 & 1+S_{y}^{\text {gyro }} & 0 \\
0 & 0 & 1+S_{z}^{\text {gyro }}
\end{array}\right)+\left(\begin{array}{c}
w_{x}^{\text {gyro }} \\
w_{y}^{\text {gyro }} \\
w_{z}^{\text {gyro }}
\end{array}\right)
$$

Where $\omega^{g}, \tilde{\omega}^{g}, b^{\text {gyro }}, M^{\text {gyro }}, S^{\text {gyro }}, w^{\text {gyro }}$ stand for reference angular rate, gyroscope sensed angular rate, bias, misalignment, scale factor and noise parameters for modeled gyroscope respectively. 


\subsection{Inertial Navigation System (INS) Dynamics}

Main objective of the inertial navigation systems is obtaining position, velocity and attitude information of the host platform. While constituting this navigation information, onboard computer of the INS makes use of inertial data collected from IMU, gravity data obtained from the Earth model and gravity model. Collected data is processed by the help of kinematic INS mechanization equations and desired inertial solution is acquired. In this section of the thesis, dynamics of the inertial navigation system is discussed in detail.

\subsubsection{Coordinate Frames}

A coordinate frame is a reference that provides an origin and a set of axis in three dimensional space for a moving object [9]. Defining required number of orthogonal and right handed frames is the fundamental process of navigation. While navigating in the vicinity of the Earth, velocity and position with respect to the Earth are the main outputs [8]. At the same time, measurements of IMU are referenced to a nonrotating inertial frame expressed in body axis of the vehicle. Therefore it is customary to define the coordinate frames before considering the navigation equations in order to prevent any confusion.

\subsubsection{Inertial Frame (I-Frame)}

Origin of the inertial frame is the centre of the Earth and its axes are non-rotating with respect to the fixed stars. Z-axis of the inertial frame is coincident with the Earth's polar axis (z-axis).

\subsubsection{Earth Frame (E-Frame)}

Origin of the Earth frame is the center of the Earth and its axes are fixed with respect to the Earth (rotating around the Earth's polar axis with Earth rate). X-axis of the 
Earth frame points to the Greenwich meridian from the center of the Earth. Its Z-axis is coincident with the Earth's polar axis. Y-axis of the Earth frame is defined along the equatorial plane forming a right handed orthogonal set with $\mathrm{X}$-axis and $\mathrm{Z}$-axis.

\subsubsection{Navigation Frame (N-Frame)}

Origin of the Navigation frame is at the center of navigation system which moves with the host platform. It is a locally level geographic frame whose $\mathrm{X}, \mathrm{Y}, \mathrm{Z}$ axes are aligned with the directions of north, east and down respectively.

\subsubsection{Body Frame (B-Frame)}

Origin of the body frame is at the center of navigation system. Orthogonal axes of the body frame are aligned with roll, pitch and yaw axes of the navigating platform. $\mathrm{X}$-axis of the body frame is forward along the longitudinal axis of the navigating platform, Z-axis is directed downward and Y-axis is defined towards right side of the navigating platform forming a right handed orthogonal set with $\mathrm{X}$-axis and $\mathrm{Z}$-axis.

\subsubsection{Earth Model}

Since velocity and position with respect to Earth are the main outputs while navigating in the vicinity of the Earth [8], it is required to define an Earth model which makes realistic assumptions regarding the shape of the Earth. In most of the navigation systems, Earth is modeled as an ellipsoid which is defined by two radii [9]. The equatorial radius Ro is defined as the length of semi-major axis which is equal to the radius of the Earth's equatorial plane. Polar radius Rp is defined as the length of semi-minor axis which is equal to the distance from earth center to either pole.

In accordance with this model, the following parameters are defined in the WGS84 standards as given below. The ellipsoid is represented by the equatorial radius and 
the flattening. Other Earth related parameters may be obtained using these two terms [15].

$$
\begin{array}{ll}
R_{0}=6,378,137.0 m & : \text { Length of semi-major axis } \\
R_{p}=R_{0}(1-f) & : \text { Length of semi-minor axis } \\
f=1 / 298.257223563 & : \text { Flattening of the ellipsoid } \\
\mathrm{e}=(f(2-f))^{1 / 2} & : \text { Major eccentricity of the ellipsoid }
\end{array}
$$

A meridian of curvature and a transverse radius of curvature, which will be used in defining the rates of change of latitude and longitude, may also be derived in accordance with the ellipsoidal definition of the Earth [8].

$$
\begin{aligned}
& R_{N}=\frac{R_{0}\left(1-e^{2}\right)}{\left(1-e^{2} \sin ^{2} L\right)^{3 / 2}}: \text { Meridian radius of curvature } \\
& R_{E}=\frac{R_{0}}{\left(1-e^{2} \sin ^{2} L\right)^{1 / 2}}: \text { Transverse radius of curvature }
\end{aligned}
$$

For navigation purposes, rotation rate of the Earth is assumed as a constant according to the WGS84 standards. Value of the Earth rate is given in the equation (2.6) [15].

$$
\omega_{i e}=\Omega=7.292115 \times 10^{-5} \mathrm{rad} / \mathrm{s}
$$

Rotation of the Earth is in counter-clockwise direction around Z-axis of E-frame. Earth rotation vector can be expressed in E-frame as follows.

$$
\omega_{I E}^{E}=\left(\begin{array}{lll}
0 & 0 & \omega_{I E}
\end{array}\right)^{T}
$$

Earth rotation vector can be expressed in $\mathrm{N}$-frame as a function of geodetic latitude.

$$
\omega_{I E}^{N}=\left(\begin{array}{lll}
\omega_{I E} \cos L & 0 & -\omega_{I E} \sin L
\end{array}\right)^{T}
$$

\subsubsection{Gravity model}

A relatively simple model of acceleration constituted by gravity at the ellipsoid as a function of latitude is given by WGS84 datum as in the Equation (2.9). This is a gravity field model called Somigliana. It is assumed that the gravity vector is 
perpendicular to the predefined ellipsoid and its direction is downwards through the third axis of the $\mathrm{N}$-frame [9].

$$
g_{0}(L) \approx 9.7803253359 \frac{\left(1+0.001931853 \sin ^{2} L\right)}{\sqrt{1-e^{2} \sin ^{2} L}} \mathrm{~m} / \mathrm{s}^{2}
$$

The variation of the gravitational field with height can be modeled using a scaling parameter which is a function of latitude and height above the ellipsoid as follows.

$$
\begin{gathered}
g(L, h) \approx \frac{\left(r_{e S}^{e}(L)\right)^{2}}{\left(r_{e S}^{e}(L)+h\right)^{2}} g_{0}(L) \\
r_{e S}^{e}(L)=R_{E} \sqrt{\cos ^{2} L+\left(1-e^{2}\right)^{2} \sin ^{2} L}: \text { Geocentric radius }
\end{gathered}
$$

Acceleration due to gravity which is also called "plumb-bob gravity" is composed of gravitational acceleration and centripetal acceleration [16].The formulas given above define magnitude of the acceleration due to gravity. Since the direction of this acceleration is downwards perpendicular to the predefined ellipsoid, vector form of the plumb-bob gravity can be represented in $\mathrm{N}$-frame as given in equation (2.12).

$$
g_{p}^{N}=\left(\begin{array}{lll}
0 & 0 & g(L, h)
\end{array}\right)^{T}
$$

\subsubsection{INS Mechanization}

This section of the thesis focuses on the equations derived for obtaining navigation solution, namely attitude, velocity and position, using linear acceleration and angular rate outputs of IMU's. Figure 5 shows a schematic of operations utilized in inertial navigation processing. 


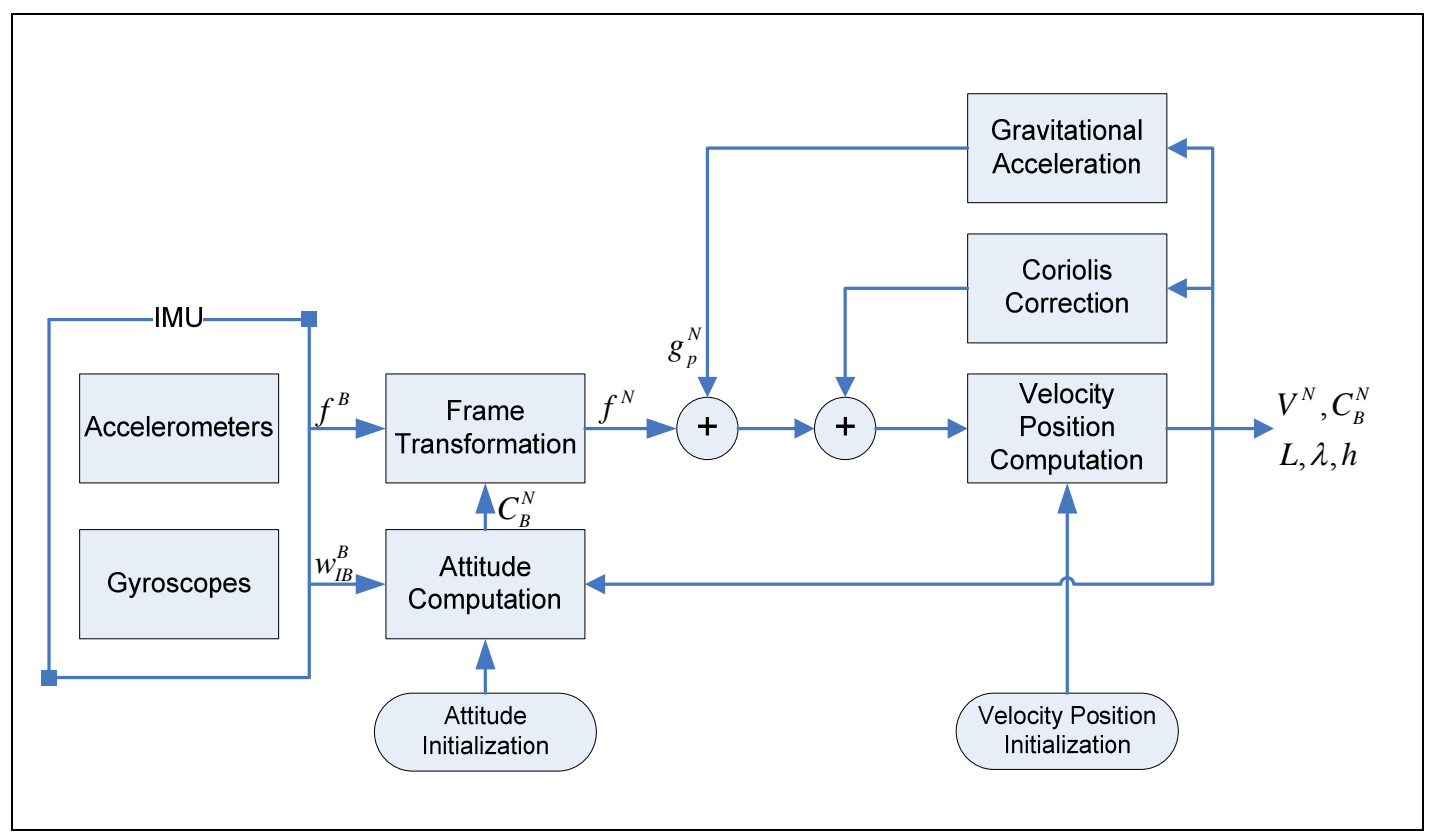

Figure 5 Schematic of the INS mechanization equations

After the initialization of navigation states, attitude computation is done in order to transform the linear acceleration outputs of accelerometers from B-frame to $\mathrm{N}$-frame. Then using gravity model and coriolis correction, velocity and position computations are done. INS mechanization is defined in the form of continuous time differential equations. Equations for attitude, velocity and position mechanizations are given in sections 2.2.4.1, 2.2.4.2 and 2.2.4.3 respectively.

\subsubsection{Attitude Mechanization}

Attitude is a significant parameter for strapdown inertial navigation processing since transformation of vectors between different reference frames is utilized by using this information. In the literature, there are miscellaneous methods for representing attitude [16]. In the context of this thesis, coordinate transformation matrix, in other words direction cosine matrix (DCM), is the selected notation for updating attitude solution. For visualization and evaluation of the results, Euler angles are computed from the DCM by a simple algorithm. 
Differential equation describing the rate of change of DCM that is used to transform vectors represented in $\mathrm{B}$-frame to vectors in $\mathrm{N}$-frame is given below [17]. The variables defined as $\left(\omega_{I B}^{B} \mathrm{x}\right),\left(\omega_{I N}^{N} \mathrm{x}\right)$ are skew-symmetric forms of the corresponding vectors.

$$
\dot{C}_{B}^{N}=C_{B}^{N}\left(\omega_{I B}^{B} \mathrm{x}\right)-\left(\omega_{I N}^{N} \mathrm{x}\right) C_{B}^{N}
$$

Angular rates existing in the equation above are defined as follows.

$$
\begin{aligned}
& \omega_{I N}^{N}=\omega_{I E}^{N}+\omega_{E N}^{N} \\
& \omega_{I E}^{N}=\left(\begin{array}{lll}
\omega_{I E} \cos L & 0 & -\omega_{I E} \sin L
\end{array}\right)^{T}: \text { : Earth rate in N-frame }
\end{aligned}
$$

Transport rate $\left(\omega_{E N}^{N}\right)$ is defined as the angular rate of the $\mathrm{N}$-frame with respect to the E-frame represented in the $\mathrm{N}$-frame.

$$
\omega_{E N}^{N}=\left(\begin{array}{lll}
\dot{\lambda} \cos L & -\dot{L} & -\dot{\lambda} \sin L
\end{array}\right)^{T}=\left(\frac{V_{E}}{R_{E}+h}-\frac{V_{N}}{R_{N}+h}-\frac{V_{E} \tan L}{R_{E}+h}\right)^{T}
$$

Where $L, \lambda$ stand for latitude, longitude and $V_{E}, V_{N}$ stand for east and north components of $V^{N}$. Initial value for this differential equation is obtained by using initial Euler angles as follows.

$$
C_{B}^{N}=\left(\begin{array}{ccc}
\cos \theta \cos \psi & -\cos \phi \sin \psi+\sin \phi \sin \theta \cos \psi & \sin \phi \sin \psi+\cos \phi \sin \theta \cos \psi \\
\cos \theta \sin \psi & \cos \phi \cos \psi+\sin \phi \sin \theta \sin \psi & -\sin \phi \cos \psi+\cos \phi \sin \theta \sin \psi \\
-\sin \theta & \sin \phi \cos \theta & \cos \phi \cos \theta
\end{array}\right)
$$

Where $\phi, \theta, \psi$ are roll, pitch and yaw angles respectively. Initial values of these angles are predefined depending on individual simulation trajectories.

\subsubsection{Velocity Mechanization}

As stated before, inertial navigation deals with the velocity of a moving object with respect to the Earth. Since position solution is expressed in curvilinear coordinates by latitude, longitude and altitude; velocity solution is supposed to be expressed in the $\mathrm{N}$-frame. At the same time, linear accelerations are measured with respect to I-frame 
and represented in B-frame. Therefore, starting from the I-frame, differential equations for velocity mechanization are derived in I-frame, E-frame and $\mathrm{N}$-frame in turn [18].

Velocity in I-frame:

$$
\dot{V}^{I}=f^{I}+g^{I}-\omega_{I E}^{I} \times\left(\omega_{I E}^{I} \times r\right)-\omega_{I E}^{I} \times V^{I}
$$

Velocity in E-frame:

$$
\dot{V}^{E}=f^{E}+g^{E}-\omega_{I E}^{E} \times\left(\omega_{I E}^{E} \times r\right)-2 \omega_{I E}^{E} \times V^{E}
$$

Velocity in N-frame:

$$
\dot{V}^{N}=f^{N}+g^{N}-\omega_{I E}^{N} \times\left(\omega_{I E}^{N} \times r\right)-\left(2 \omega_{I E}^{N}+\omega_{E N}^{N}\right) \times V^{N}
$$

By substituting $f^{N}=C_{B}^{N} f^{B}$ and plumb-bob gravity vector, conclusive equation for $\mathrm{N}$-frame velocity mechanization is obtained.

$$
\dot{V}^{N}=C_{B}^{N} f^{B}+g_{p}^{N}-\left(2 \omega_{I E}^{N}+\omega_{E N}^{N}\right) \times V^{N}
$$

\subsubsection{Position Mechanization}

Position solution of inertial navigation systems is generally expressed in curvilinear coordinates by latitude, longitude and altitude for its simplicity and comprehensibility. The rate of change of the curvilinear position can be expressed as follows [19].

$$
\dot{L}=\frac{V_{N}}{R_{N}+h} ; \dot{\lambda}=\frac{V_{E}}{R_{E}+h} ; \dot{h}=-V_{D}
$$

Where; $V_{N}, V_{E}, V_{D}$ stand for the north, east and down components of the $\mathrm{N}$-frame velocity vector respectively. Definitions of $R_{N}, R_{E}$ are given in the Earth model equation (2.5). 
In order to transform vectors defined in the N-frame to E-frame, a DCM is formed which is function of latitude and longitude. For instance, the Earth rate vector represented in E-frame is transformed to the $\mathrm{N}$-frame via transpose of this DCM [19].

$$
C_{N}^{E}=\left(\begin{array}{ccc}
-\sin \lambda \cos \lambda & -\sin \lambda & -\cos L \cos \lambda \\
-\sin L \sin \lambda & \cos \lambda & -\cos L \sin \lambda \\
\cos L & 0 & -\sin L
\end{array}\right)
$$

A summary of the INS mechanization equations is given in the table below.

Table 1 INS mechanization equations

\begin{tabular}{|l|c|}
\hline Attitude & $\dot{C}_{B}^{N}=C_{B}^{N}\left(\omega_{I B}^{B} \mathrm{x}\right)-\left(\omega_{I N}^{N} \mathrm{x}\right) C_{B}^{N}$ \\
\hline Velocity & $\dot{V}^{N}=C_{B}^{N} f^{B}+g_{p}^{N}-\left(2 \omega_{I E}^{N}+\omega_{E N}^{N}\right) \times V^{N}$ \\
\hline Position & $\dot{L}=\frac{V_{N}}{R_{N}+h} ; \dot{\lambda}=\frac{V_{E}}{R_{E}+h} ; \dot{h}=-V_{D}$ \\
\hline
\end{tabular}

\subsubsection{INS Error Model}

In the context of this thesis, an error state system model will be constituted for the integration of INS and GPS. Position, velocity, attitude errors and inertial sensor biases are selected as the state space variables. Therefore nonlinear equations for position, velocity and attitude given in the previous section need to be linearized about the most recent navigation solution. For this linearization operation, nonlinear equations are perturbed for the state space variables [9]. In the derivation step of this model, second order terms in the error quantities are dropped [19].

\subsubsection{Attitude Error Model}

For the attitude errors, small angle attitude error model is utilized. The attitude mechanization equation [equation (2.13)] is linearized with respect to the state space variables and the following results are obtained [9]. 


$$
\delta \dot{\Psi}=-\omega_{I N}^{N} \times \delta \Psi+\delta \omega_{I N}^{B}-C_{B}^{N} \delta \omega_{I B}^{B}
$$

Where; $(\delta \Psi)$ is the attitude error present in the state estimate.

$\left(\delta \omega_{I B}^{B}\right)$ is the gyroscope error vector represented in the B-frame.

$\left(\delta \omega_{I N}^{N}\right)$ is the error in the angular rate of the $\mathrm{N}$-frame with respect to the I-frame which is composed of earth rate error and transport rate error as given below.

$$
\delta \omega_{I N}^{N}=\delta \omega_{I E}^{N}+\delta \omega_{E N}^{N}
$$

Earth rate error $\left(\delta \omega_{I E}^{N}\right)$ is obtained by perturbing the Earth rate $\left(\omega_{I E}^{N}\right)$ by latitude error $(\delta L)$. Assuming that perturbation of the Earth rate is equal to zero

$$
\delta \omega_{I E}^{N}=(-\Omega \sin L \delta L \quad 0 \quad-\Omega \cos L \delta L)^{T}
$$

Transport rate error $\left(\delta \omega_{E N}^{N}\right)$ is similarly obtained by perturbing $\left(\omega_{E N}^{N}\right)$ by four of the state space variables $\delta V_{E}, \delta V_{N}, \delta h, \delta L$.

$$
\delta \omega_{E N}^{N}=\left(\begin{array}{l}
\frac{\delta V_{E}}{\left(R_{E}+h\right)}-\frac{V_{E}}{\left(R_{E}+h\right)^{2}} \delta h \\
-\frac{\delta V_{N}}{\left(R_{N}+h\right)}+\frac{V_{N}}{\left(R_{N}+h\right)^{2}} \delta h \\
\frac{-\tan L}{\left(R_{E}+h\right)} \delta V_{E}+\frac{V_{E} \tan L}{\left(R_{E}+h\right)^{2}} \delta h-\frac{V_{E}}{\left(R_{E}+h\right) \cos ^{2} L} \delta L
\end{array}\right)
$$

Attitude error model can be represented in state space form as follows. Definitions for the elements of the state space are given in Section 2.2.5.4.

$$
\delta \dot{\Psi}=\left(\begin{array}{lll}
F_{\Psi P} & F_{\Psi V} & F_{\Psi \Psi}
\end{array}\right)\left(\begin{array}{c}
\delta P \\
\delta V \\
\delta \Psi
\end{array}\right)-C_{B}^{N} \delta \omega_{I B}^{B}
$$




\subsubsection{Velocity Error Model}

Similarly, velocity error model is obtained by perturbing the velocity mechanization equation given in the equation (2.20) by state vectors, Earth rate and transport rate errors. The rate of change of velocity error is given below as a function of state variables and gravity error [9].

$$
\delta \dot{V}^{N}=-\Psi C_{B}^{N} f^{B}+C_{B}^{N} \delta f^{B}-\left(2 \omega_{I E}^{N}+\omega_{E N}^{N}\right) \times \delta V^{N}-\left(2 \delta \omega_{I E}^{N}+\delta \omega_{E N}^{N}\right) \times V^{N}-\frac{2 g_{0}(L)}{r_{e S}^{e}(L)} \delta h
$$

Velocity error model can be represented in state space form as follows. Definitions for the elements of the state space are given in section 2.2.5.4 state space error model.

$$
\delta \dot{V}^{N}=F_{V P} \delta P+F_{V V} \delta V+F_{V \Psi} \delta \Psi
$$

\subsubsection{Position Error Model}

Analogous to velocity error model, position error model is obtained by perturbing the position mechanization equation given in the equation (2.21) by position and velocity error states [9].

$$
\begin{aligned}
& \delta \dot{L}=\frac{1}{\left(R_{N}+h\right)} \delta V_{N}-\frac{V_{N}}{\left(R_{N}+h\right)^{2}} \delta h \\
& \delta \dot{\lambda}=\frac{1}{\left(R_{E}+h\right) \cos L} \delta V_{E}+\frac{V_{E} \tan L}{\left(R_{E}+h\right) \cos L} \delta L-\frac{V_{E}}{\left(R_{E}+h\right)^{2} \cos L} \delta h \\
& \delta \dot{h}=-\delta V_{D}
\end{aligned}
$$

Position error model can be represented in state space form as follows. Definitions for the elements of the state space are given in section 2.2.5.4 state space error model.

$$
\delta \dot{P}=\left(\begin{array}{lll}
F_{P P} & F_{P V} & F_{P \Psi}
\end{array}\right)\left(\begin{array}{l}
\delta P \\
\delta V \\
\delta \Psi
\end{array}\right)
$$




\subsubsection{State Space Error Model}

State space for the INS/GPS integration process is composed of fifteen elements namely position, velocity and attitude errors, accelerometer biases and gyroscope biases. Each of these states is a three dimensional vector. This state space model will be utilized as the basis for linearized system model of the estimators of INS/GPS integration process. State transition matrix is obtained by using the state space forms of individual error models for each state space variable as follows.

$$
\left(\begin{array}{c}
\delta \dot{P} \\
\delta \dot{V} \\
\delta \dot{\psi} \\
\delta \dot{a}_{\text {Bias }} \\
\delta \dot{\omega}_{\text {Bias }}
\end{array}\right)=\left(\begin{array}{ccccc}
F_{P P} & F_{P V} & F_{P \psi} & 0 & 0 \\
F_{V P} & F_{V V} & F_{V \psi} & C_{B}^{N} & 0 \\
F_{\psi P} & F_{\psi V} & F_{\psi \psi} & 0 & C_{B}^{N} \\
0 & 0 & 0 & 0 & 0 \\
0 & 0 & 0 & 0 & 0
\end{array}\right)\left(\begin{array}{c}
\delta P \\
\delta V \\
\delta \psi \\
\delta a_{\text {Bias }} \\
\delta \omega_{\text {Bias }}
\end{array}\right)
$$

Components of this state transition matrix are given in the equations (2.33) to (2.41) $[9]$

$$
\begin{aligned}
& F_{P P}=\left(\begin{array}{ccc}
0 & 0 & \frac{-V_{N}}{\left(R_{N}+h\right)^{2}} \\
\frac{V_{E} \tan L}{\left(R_{E}+h\right) \cos L} & 0 & \frac{-V_{E}}{\left(R_{E}+h\right)^{2} \cos L} \\
0 & 0 & 0
\end{array}\right) \\
& F_{P V}=\left(\begin{array}{ccc}
\frac{1}{\left(R_{N}+h\right)} & 0 & 0 \\
0 & \frac{1}{\left(R_{E}+h\right) \cos L} & 0 \\
0 & 0 & -1
\end{array}\right) \\
& F_{P \Psi}=0_{3 \times 3}
\end{aligned}
$$




$$
\begin{aligned}
& F_{V P}=\left(\begin{array}{ccc}
-\frac{V_{E}^{2}}{\left(R_{E}+h\right) \cos ^{2} L}-2 V_{E} \Omega \cos L & 0 & \frac{V_{E}^{2} \tan L}{\left(R_{E}+h\right)^{2}}-\frac{V_{N} V_{D}}{\left(R_{N}+h\right)^{2}} \\
\frac{V_{N} V_{E}}{\left(R_{E}+h\right) \cos ^{2} L}+2 V_{N} \Omega \cos L-2 V_{D} \Omega \sin L & 0 & -\frac{V_{E} V_{N} \tan L+V_{E} V_{D}}{\left(R_{E}+h\right)^{2}} \\
2 V_{E} \Omega \sin L & 0 & \frac{V_{E}^{2}}{\left(R_{E}+h\right)^{2}}+\frac{V_{N}^{2}}{\left(R_{N}+h\right)^{2}}
\end{array}\right) \\
& F_{V V}=\left(\begin{array}{ccc}
\frac{V_{D}}{\left(R_{N}+h\right)} & -\frac{2 V_{E} \tan L}{\left(R_{E}+h\right)}-2 \Omega \sin L & \frac{V_{N}}{\left(R_{N}+h\right)} \\
\frac{V_{E} \tan L}{\left(R_{E}+h\right)}+2 \Omega \sin L & \frac{V_{N} \tan L+V_{D}}{\left(R_{E}+h\right)} & \frac{V_{E}}{\left(R_{E}+h\right)}+2 \Omega \cos L \\
-\frac{2 V_{N}}{\left(R_{N}+h\right)} & -\frac{2 V_{E}}{\left(R_{E}+h\right)}-2 \Omega \cos L & 0
\end{array}\right) \\
& F_{V \Psi}=\left(\begin{array}{ccc}
0 & -f_{D} & f_{E} \\
f_{D} & 0 & -f_{N} \\
-f_{E} & f_{N} & 0
\end{array}\right) ;\left(\begin{array}{l}
f_{N} \\
f_{E} \\
f_{D}
\end{array}\right)=C_{B}^{N}\left(\begin{array}{l}
f_{x} \\
f_{y} \\
f_{z}
\end{array}\right) \\
& F_{\Psi P}=\left(\begin{array}{ccc}
\Omega \sin L & 0 & \frac{V_{E}}{\left(R_{E}+h\right)^{2}} \\
0 & 0 & -\frac{V_{N}}{\left(R_{N}+h\right)^{2}} \\
\Omega \cos L+\frac{V_{E}}{\left(R_{E}+h\right) \cos ^{2} L} & 0 & -\frac{V_{E} \tan L}{\left(R_{E}+h\right)^{2}}
\end{array}\right) \\
& F_{\Psi V}=\left(\begin{array}{ccc}
0 & -\frac{1}{\left(R_{E}+h\right)} & 0 \\
\frac{1}{\left(R_{N}+h\right)} & 0 & 0 \\
0 & \frac{\tan L}{\left(R_{E}+h\right)} & 0
\end{array}\right) ; F_{\Psi \Psi}=\left(\begin{array}{ccc}
0 & \omega_{D} & -\omega_{E} \\
-\omega_{D} & 0 & \omega_{N} \\
\omega_{E} & -\omega_{N} & 0
\end{array}\right)
\end{aligned}
$$




$$
\begin{gathered}
\text { Where }\left(\begin{array}{lll}
\omega_{N} & \omega_{E} & \omega_{D}
\end{array}\right)^{T}=\omega_{I N}^{N}=\omega_{I E}^{N}+\omega_{E N}^{N} \\
F_{\Psi \Psi}=\left(\begin{array}{ccc}
0 & -\frac{V_{E} \sin L}{\left(R_{E}+h\right) \cos L}-\Omega \sin L & \frac{V_{N}}{R_{N}+h} \\
\frac{V_{E} \sin L}{\left(R_{E}+h\right) \cos L}+\Omega \sin L & 0 & \frac{V_{E}}{\left(R_{E}+h\right)}+\Omega \cos L \\
-\frac{V_{N}}{R_{N}+h} & -\frac{V_{E}}{\left(R_{E}+h\right)}-\Omega \cos L & 0
\end{array}\right)
\end{gathered}
$$




\section{CHAPTER 3}

\section{GLOBAL POSITIONING SYSTEM (GPS)}

The global positioning system is a satellite based navigation system that provides the users three dimensional position and velocity solution by using passive radio signals. Accurate time information is also provided in addition to the navigation solution when there is an unobstructed line of sight to four or more satellites. GPS project was developed in order to get through the deficiencies of the previous navigation systems in 1973 by U.S. Department of Defense. The system became fully operational in 1994 and made freely available for the civilian use [3]. Several improvements and modernizations are made to the system in order to meet the military and civilian users' needs. There are ongoing studies which aim to increase performance and accuracy of the system [10].

The basic operation of the GPS is obtaining user position and velocity using radio navigation signals broadcast by the orbiting satellites. These signals include the position and velocity information of each satellite and the time of transmission of the signal from the satellite. By comparing the transmission and receiving times of the GPS signals, the range between satellites and the GPS receiver antenna is measured assuming that the signal travels with the speed of light. Geometrically, position of the user should be on a sphere centered at the corresponding satellite with a radius defined by the range information obtained. Each ranging data gathered from individual satellites defines a similar sphere for the position of the user. When two satellites are available, position solution is confined on a circle formed by the intersection of two spheres. By using the information from a third satellite, two 
possible points are determined to define the user position. Therefore, signals from at least four satellites are required in order to obtain navigation solution of a GPS user. When there exists an obstruction of line of sight to the satellites or a signal loss resulting in access to less than four satellites, standalone GPS cannot provide navigation solution to its users [19].

In this chapter, fundamental characteristics of the GPS are considered first. Then techniques for obtaining the GPS navigation solution for position are investigated. After defining the sources of measurement errors, a nonlinear measurement model is obtained at the end of the chapter.

\subsection{GPS Fundamentals}

GPS consists of three main segments namely the space segment, the control segment and the user segment. The space and control segments are maintained and operated by the US Air Force [20]. The user segment consists of GPS receiver and receiver antenna which are commercial off-the-shelf products.

The space segment is composed of the GPS satellites which rotate in Earth centered approximately circular orbits. The set of satellites in orbit providing the ranging signals and data messages to the user segment is called the satellite constellation. Standard constellation consists of twenty-four satellites which are positioned in six orbital planes with four satellites in each plane [21]. The orbits are equally spaced around the equatorial plane with a nominal inclination angle which provides an allday long global navigation capability. The subsystems of the satellites perform some functions such as maintaining the solar panels pointing to the sun and satellites pointing to the Earth [21].

The control segment is physically composed of the master control station, monitor stations and the ground antennas. Basic operation of the control segment is maintaining the orbital configuration of satellites, monitoring the health of satellites and signals broadcast. Moreover, satellite clock corrections, ephemerides, almanac 
and other control parameter updates are sent to the constellation at least once per day by the control segment. Command and control of the GPS constellation is provided by the master control station centrally. All of the six monitor stations and four ground antennas are also controlled remotely by the master control station [20].

The user segment comprises the GPS receiver and also the receiver antenna. The radio frequency signals transmitted from GPS constellation are converted to electronic signals by receiver antenna and processed by the receiver in order to obtain position, velocity and time information. A GPS receiver processor consists of two main components. First one is the ranging processor which determines the transmission time of the received signal and forms the GPS observables. Second one is the navigation processor which obtains GPS navigation solution by using the GPS observables [9]. In the next section, basic operations used in the navigation processor are summarized.

\subsection{GPS Navigation Solution}

In order to calculate the position of a GPS receiver, at least four satellites should be available since there are four parameters to be determined to find the user position in the E-frame; three parameters for $\mathrm{X}, \mathrm{Y}, \mathrm{Z}$ coordinates and receiver clock bias term. In order to obtain these four unknowns four independent equations are needed. Position calculations are done by using the ranging signals and data messages which are acquired from individual GPS signals broadcast by the satellites [19].

Position computation of the receiver is done by making use of the pseudo range observable which is obtained from the ranging signals. Pseudo range is the receiver calculated erroneous range between each satellite and the receiver antenna. It is obtained by comparing the transmission and receiving time of the GPS signals and multiplying by the speed of light [22].

Pseudo ranges can be modeled as: 


$$
P_{i}=\left(t_{r}-t_{i}\right) c=\tau_{i} C
$$

Where; i stands for the number of satellite, $\tau_{i}$ is the travelling time of the signal from satellite $\mathrm{i}$ to the receiver, and $c$ represents speed of light.

Atomic clocks in each satellite and clocks in the receivers do not run exactly aligned with the true GPS time which is represented by $t^{G P S}$. There exists a clock offset in each clock of the satellites and the receiver. Clock offsets of the GPS satellites are determined by the control segments and sent to each satellite. This correction term is present in the data message broadcast by each satellite [9].

The clock offsets of GPS satellites and the receiver can be defined as:

$$
\begin{aligned}
& t_{i}=t^{G P S}+\delta t_{i}: i^{\prime} \text { th satellite time } \\
& t_{r}=t^{G P S}+\delta t_{r}: \text { receiver time }
\end{aligned}
$$

\subsubsection{Measurement Equation}

The most common algorithm used in the position calculations of receivers using pseudo ranges is the least squares estimator (LSE) [23]. This method can easily be used for over determined systems where the number of equations is more than the number of unknowns. First of all, measurement equation for the GPS position should be written in order to use LSE.

The geometric range between the satellites and the receiver can be defined as:

$$
\rho_{i}=\sqrt{\left(X_{i}-X\right)^{2}+\left(Y_{i}-Y\right)^{2}+\left(Z_{i}-Z\right)^{2}}
$$

Where $X_{i}, Y_{i}, Z_{i}$ and $X, Y, Z$ correspond to ECEF position of the $\mathrm{i}^{\text {th }}$ satellite and true position of the GPS receiver respectively. ECEF positions and velocities of the satellites are present in the data message broadcast by each satellite therefore these variables are given. 
By adding the measurement errors of the GPS receiver to the geometric range, equation for the measured pseudo range can be obtained as follows:

$$
P_{i}=\rho_{i}+c\left(\delta t_{r}-\delta t_{i}\right)+T_{i}+I_{i}+e_{i}
$$

Undefined variables in the measured pseudo range equation above can be clarified as $T_{i}$ : Tropospheric delay

$I_{i}:$ Ionospheric delay

$e_{i}$ : Pseudo range measurement residual

Atmospheric delay parameters are included in the data message broadcast by each satellite as correction terms. Definitions of these measurement error parameters are given in detail in section 3.3.

Combining the geometric range equation with the previous equation, conclusive expression for the measured pseudo range can be obtained as follows.

$$
P_{i}=\sqrt{\left(X_{i}-X\right)^{2}+\left(Y_{i}-Y\right)^{2}+\left(Z_{i}-Z\right)^{2}}+c\left(\delta t_{r}-\delta t_{i}\right)+T_{i}+I_{i}+e_{i}
$$

There are only four unknowns in the equation above, namely receiver position $X, Y, Z$ and receiver clock bias $\delta t_{r}$.

\subsubsection{Least Squares Estimate of The Navigation Solution}

Measurement residual term $e_{i}^{k}$ should be minimized using the least square estimator for obtaining the unknown receiver position. In order to use this estimator, the nonlinear equation for the pseudo range measurement needs to be linearized for receiver position $X, Y, Z$ coordinates and receiver clock bias as follows.

Starting point of the linearization can be chosen to be the center of the Earth as a rule of thumb [23].

$$
\left(X_{0}, Y_{0}, Z_{0}\right)=(0,0,0)
$$


The estimated user position is used as the linearization point of the next iteration. After a number of iterations using the same GPS measurements, user navigation solution is obtained.

Define geometric range function as,

$$
f(X, Y, Z)=\sqrt{\left(X_{i}-X\right)^{2}+\left(Y_{i}-Y\right)^{2}+\left(Z_{i}-Z\right)^{2}}
$$

Define $\left(X_{1}, Y_{1}, Z_{1}\right)=\left(X_{0}+\Delta X, Y_{0}+\Delta Y, Z_{0}+\Delta Z\right)$

First order Taylor series expansion of the function can be obtained as follows:

$$
f\left(X_{1}, Y_{1}, Z_{1}\right)=f\left(X_{0}, Y_{0}, Z_{0}\right)+\frac{\partial f\left(X_{0}, Y_{0}, Z_{0}\right)}{\partial X_{0}} \Delta X+\frac{\partial f\left(X_{0}, Y_{0}, Z_{0}\right)}{\partial Y_{0}} \Delta Y+\frac{\partial f\left(X_{0}, Y_{0}, Z_{0}\right)}{\partial Z_{0}} \Delta Z
$$

After taking the partial derivatives, the first order linearized equation is obtained as:

$$
P_{i}=\rho_{i}-\frac{X_{i}-X_{0}}{\rho_{i}} \Delta X-\frac{Y_{i}-Y_{0}}{\rho_{i}} \Delta Y-\frac{Z_{i}-Z_{0}}{\rho_{i}} \Delta Z+c\left(\delta t_{r}-\delta t_{i}\right)+c \Delta t_{r}+T_{i}+I_{i}+e_{i}
$$

Where $\rho_{i}=\sqrt{\left(X_{i}-X\right)^{2}+\left(Y_{i}-Y\right)^{2}+\left(Z_{i}-Z\right)^{2}}$

The equation for pseudo ranges can be written in matrix from as;

$$
P_{i}=\rho_{i}+\left[\begin{array}{llll}
-\frac{X_{i}-X}{\rho_{i}} & -\frac{Y_{i}-Y}{\rho_{i}} & -\frac{Z_{i}-Z}{\rho_{i}} & 1
\end{array}\right]\left[\begin{array}{c}
\Delta X \\
\Delta Y \\
\Delta Z \\
c \Delta t_{r}
\end{array}\right]+c\left(\delta t_{r}-\delta t_{i}\right)+T_{i}+I_{i}+e_{i}
$$

Rearranging this equation in the standard form for $\operatorname{LSE}(A x=b)$, the following result is obtained. In this equation, $\mathrm{n}$ stands for the number of satellites. Therefore $\mathrm{A}$ is a $(n \times 4)$ matrix [23]. 


$$
\left[\begin{array}{cccc}
-\frac{X_{1}-X}{\rho_{1}} & -\frac{Y_{1}-Y}{\rho_{1}} & -\frac{Z_{1}-Z}{\rho_{1}} & 1 \\
\vdots & \vdots & \vdots & \vdots \\
-\frac{X_{n}-X}{\rho_{n}} & -\frac{Y_{n}-Y}{\rho_{n}} & -\frac{Z_{n}-Z}{\rho_{n}} & 1
\end{array}\right]\left[\begin{array}{c}
\Delta X \\
\Delta Y \\
\Delta Z \\
c \Delta t_{r}
\end{array}\right]=\left[\begin{array}{c}
P_{1}-\rho_{1}-c \delta t_{r}+c \delta t_{1}-T_{1}-I_{1}-e_{1} \\
\vdots \\
P_{n}-\rho_{n}-c \delta t_{r}+c \delta t_{n}-T_{n}-I_{n}-e_{n}
\end{array}\right]
$$

Note that, least squares estimation for the system $(A x=b)$ is

$$
\min \left\|e_{i}\right\|^{2}=\min (b-A x)^{T}(b-A x) \Rightarrow x=\left(A^{T} A\right)^{-1} A^{T} b
$$

\subsection{Measurement Errors}

The essential measurements made by the GPS receivers are pseudo range and pseudo range rates. These measurements are subject to time-correlated and noise like errors [9]. Each of these errors will be defined and their effects to the GPS navigation solution will be discussed in this section.

The satellite clock errors originate from the oscillator noise of the precise atomic clocks. This source of errors can be calibrated by using the coefficients in the data message. The residual clock error which depends on the stability of the satellite clock and control segment's monitoring network results in about 1 meter of range error on the average [24].

The ephemeris prediction errors arise due to the errors in the control segment's prediction of the satellite positions. The effects of ephemeris errors vary with different satellite geometries, but it results in an average range error of 0.45 meter [9].

The ionosphere layer of the atmosphere refracts GPS signals due to its dispersive medium. This refraction causes a delay in the carrier of the GPS signal. The effect of ionospheric delay on range error changes with the time of the day and satellite geometry. 
Troposphere is a nondispersive layer of atmosphere which extends about $12 \mathrm{~km}$ above the Earth surface. The tropospheric delay can be corrected by using models which are functions of elevation and height of the user. These corrections of the troposhperic delay give up residual errors resulting in about $0.2 \mathrm{~m}$ of range error in pseudo range measurements [9].

Multipath error occurs when the GPS antenna receives reflected signals from a given satellite in addition to the direct signals. In most cases, GPS signals are reflected from the host body, ground, buildings and trees. These signals are always delayed compared to the direct signals.

Modeling and evaluation of these separate error sources are not in the context of this thesis. Therefore, measurement errors of GPS are modeled as additive zero mean Gaussian noise for simplicity. The resulting measurement equation is as follows.

$$
y=h(x)+v_{k}
$$

Where $v_{k} \sim N\left(0, \sigma^{2}\right)$ and $h(x)$ is the nonlinear measurement function defined in the next section. Designation of the variance of the measurement noise $\left(\sigma^{2}\right)$ is considered in section 4.3 .

\subsection{Nonlinear Measurement Model}

In the tightly coupled INS/GPS integration architecture, which is discussed in Section 4.2.2, pseudo range and pseudo range rate observables are used as GPS measurements. The nonlinear equations relating the measurements to the state variables are the geometric range and range rate functions.

$$
\begin{gathered}
h_{1}(X, Y, Z)=\sqrt{\left(X_{i}-X\right)^{2}+\left(Y_{i}-Y\right)^{2}+\left(Z_{i}-Z\right)^{2}} \\
h_{2}\left(V_{X}, V_{Y}, V_{Z}\right)=\sqrt{\left(V_{X_{i}}-V_{X}\right)^{2}+\left(V_{Y_{i}}-V_{Y}\right)^{2}+\left(V_{Z_{i}}-V_{Z}\right)^{2}}
\end{gathered}
$$


Where $\left(X_{i}, Y_{i}, Z_{i}\right)$ and $\left(V_{X_{i}}, V_{Y_{i}}, V_{Z_{i}}\right)$ correspond to ECEF position and velocity of the $\mathrm{i}^{\text {th }}$ satellite. This information is acquired from the GPS data signal for each visible satellite. $(X, Y, Z)$ and $\left(V_{X}, V_{Y}, V_{Z}\right)$ correspond to the estimated user ECEF position and velocity. The estimated navigation solution can be obtained after the feedback step involved in the error state Kalman filter implementation. 


\section{CHAPTER 4}

\section{INS/GPS INTEGRATION}

Inertial navigation systems exhibit relatively low noisy outputs which tend to drift over time. A typical aviation grade INS errors grow at rates about 1-10 nmi/h (1.8-18 $\mathrm{km} / \mathrm{h}$ ) during normal operation [25]. Contrary to INS, GPS outputs are relatively noisy but do not exhibit long-term drift [4]. Using both of these systems results in a superior navigation performance than either alone. Integrating the outputs from each sensor results in a system which can be visualized as a drift free INS. Benefits of integrating these two navigation systems can be realized by first considering advantages and disadvantages of individual systems. In section 4.1, benefits and drawbacks of two systems are discussed in detail. The subsequent section focuses on the INS/GPS integration architectures, namely loosely coupled, tightly coupled and deeply coupled architectures. Each of these architectures determines the amount of interaction of two separate systems and the attribute of data used in the integration process.

\subsection{Benefits and Drawbacks of Each System}

Each of INS and GPS has a number of advantages and disadvantages. INS operation is not dependent on outer signals or systems. It is a self-contained system operating continuously in all external conditions and provides high bandwidth output at high rates. Outputs of the INS, namely position, velocity and attitude, exhibit low shortterm noise. INS also provides linear acceleration and angular rates which are used by stabilization and flight control systems [4]. On the other hand, as the instrument 
errors are continuously integrated in the INS mechanization equations, accuracy of the navigation solution degrades with time. For a relatively long operation periods of navigation, in the order of minutes, high performance INS's are required which have high cost about $100000 \$$, high power consumption and large size restricting the platform settlement [9].

In contrast to the INS, GPS is dependent on the signals broadcast by the satellites. External conditions, namely weather conditions, visibility of the satellites, satellite geometry, multipath of the signals and jamming have significant effects on the performance of the navigation solution. Standalone GPS provides high long-term position accuracy with limited errors to a few meters. Navigation solution of the GPS includes just position and velocity of the platform. These navigation states are output at very low rates, around $1-10 \mathrm{~Hz}$, and exhibit short-term high frequency noise. Attitude solution, linear acceleration and angular rate signals are not provided by this system. GPS signals are also subject to obstruction and interference which make the system unreliable for continuous navigation. The benefits and drawbacks of the INS and standard GPS are summarized in the following table for comparison [8].

Table 2 Benefits and drawbacks of INS and GPS

\begin{tabular}{|l|l|l|}
\hline Technical Properties & INS & GPS \\
\hline $\begin{array}{l}\text { Error propagation with } \\
\text { time }\end{array}$ & Increases with time & Limited errors in time \\
\hline Attitude Solution & Available & Not availble \\
\hline Flight control signals & Available & Not availble \\
\hline Data rate & High rate $(100-1000 \mathrm{~Hz})$ & Low rate $(1-10 \mathrm{~Hz})$ \\
\hline Bandwidth & High bandwidth & Low bandwidth \\
\hline Interference & Resistent & Vulnerable \\
\hline Dependency to environment & $\begin{array}{l}\text { Independent of the outer } \\
\text { world }\end{array}$ & $\begin{array}{l}\text { Environment } \\
\text { dependent }\end{array}$ \\
\hline Initial value assignment & Not available & Available \\
\hline
\end{tabular}




\subsection{Integration Architectures}

There are three main points that determine the architecture of INS/GPS integrated navigation systems namely feedback structure, types of measurements and aiding of GPS receiver. Although these aspects are largely independent of each other, there exist three widely used definitions for the integration architecture.

Feedback structure is the way that the estimated error states are used in the navigation solution. Either closed loop or open loop feedback can be used in the INS/GPS integration process. The choice of feedback structure mainly depends on the quality of inertial sensors. Since low cost MEMS inertial sensors are utilized in the simulations, a closed-loop feedback structure is applied. In this structure, integrated navigation solution is obtained by correcting the INS navigation solution using the estimated error states and the INS solution is updated with the corrected navigation solution at each time step. This architecture eliminates the accumulation of errors in the INS solution.

In sections 4.2.1 to 4.2.3, most widely used architectures for INS/GPS integration are discussed and compared to each other in performance, simplicity and robustness aspects.

\subsubsection{Loosely Coupled}

Loosely coupled integration is most widely used architecture due to its simplicity and redundancy. Simplicity of this architecture stems from the fact that it treats INS and GPS as separated systems which produce their standalone navigation solutions as shown in Figure 6 


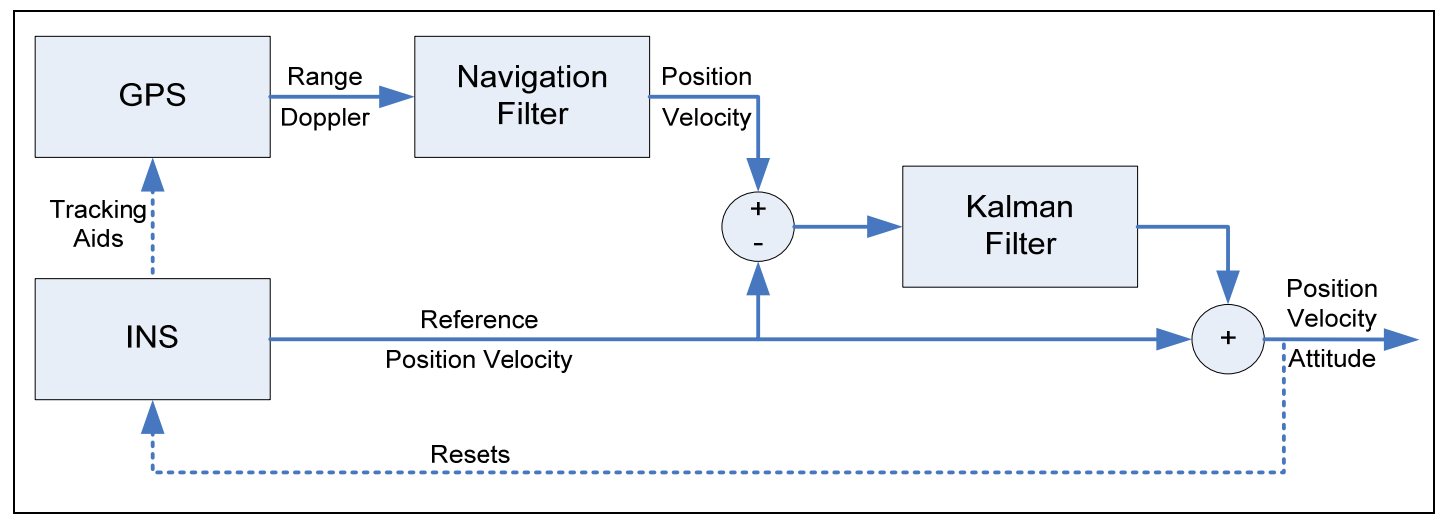

Figure 6 Flowchart of loosely coupled architecture

Position and/or velocity information computed by GPS navigation filter is used as the measurement of the integration filter in order to estimate the INS errors. Standalone INS solution is corrected using the estimated error states and integrated navigation solution is obtained

This architecture can be utilized with any commercial off-the-shelf INS and GPS user equipment. Redundancy of this architecture stems from the fact that, in addition to the integrated solution, each of the INS and GPS produces its independent solution. This enables integrity monitoring of the utilized INS/GPS Kalman filter [12].

\subsubsection{Tightly Coupled}

Tightly coupled integration eliminates the usage of cascaded filters unlike loosely coupled integration. Therefore, correlation of the measurements in GPS navigation filter is prevented. Instead of navigation solution of GPS, pseudo range and/or pseudo range rates obtained from Doppler data is used as the measurements of INS/GPS integration Kalman filter. Choice of these measurements brings nonlinearity to the measurement model of the integration filter. Figure 7 shows the flowchart of this architecture. 


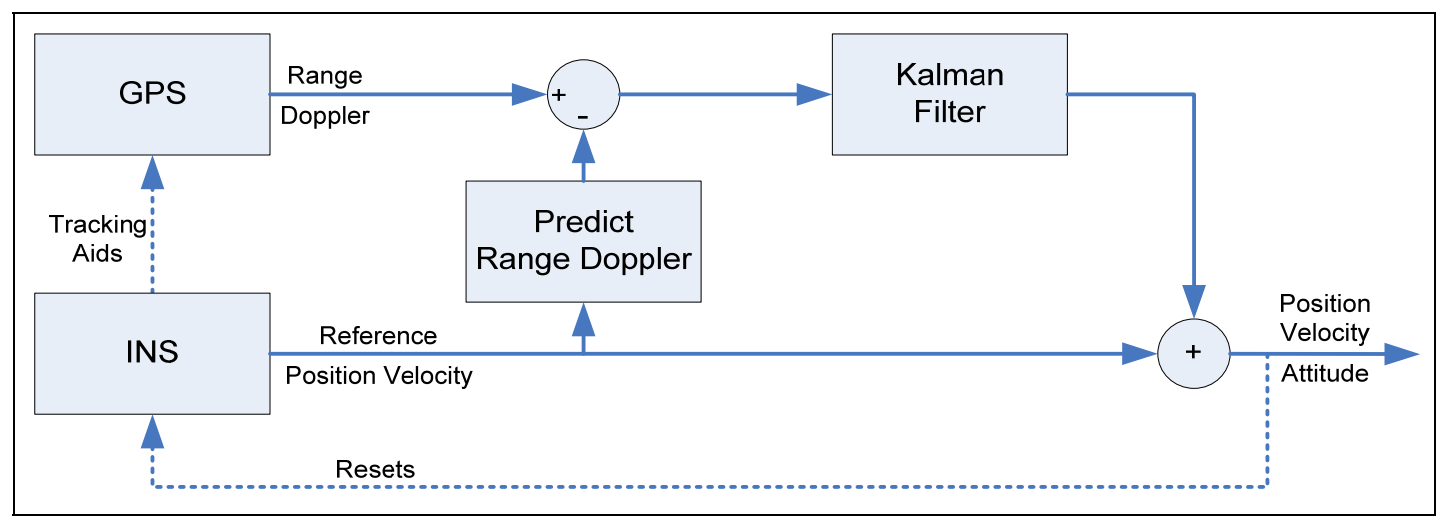

Figure 7 Flowchart of tightly coupled architecture

Similar to the loosely coupled case, integration filter estimates the errors of INS navigation solution. Integrated navigation solution is obtained by correcting the INS output by the state estimate. Furthermore, this system does not require a full GPS solution to aid the INS. Even when less than four satellites are available, integration filter will keep on operating. Compared to the loosely coupled case, tightly coupled integration has better performance and it is more robust given the same inertial instruments and GPS receiver [3].

\subsubsection{Ultra Tightly Coupled}

Ultra tightly coupled integration is more complex and most beneficial architecture which handles INS/GPS integration at the GPS tracking loop level. Traditional structure of the tracking loops is adjusted by the usage of inertial data [3]. Figure 8 shows the flowchart of an ultra tightly coupled integration architecture.

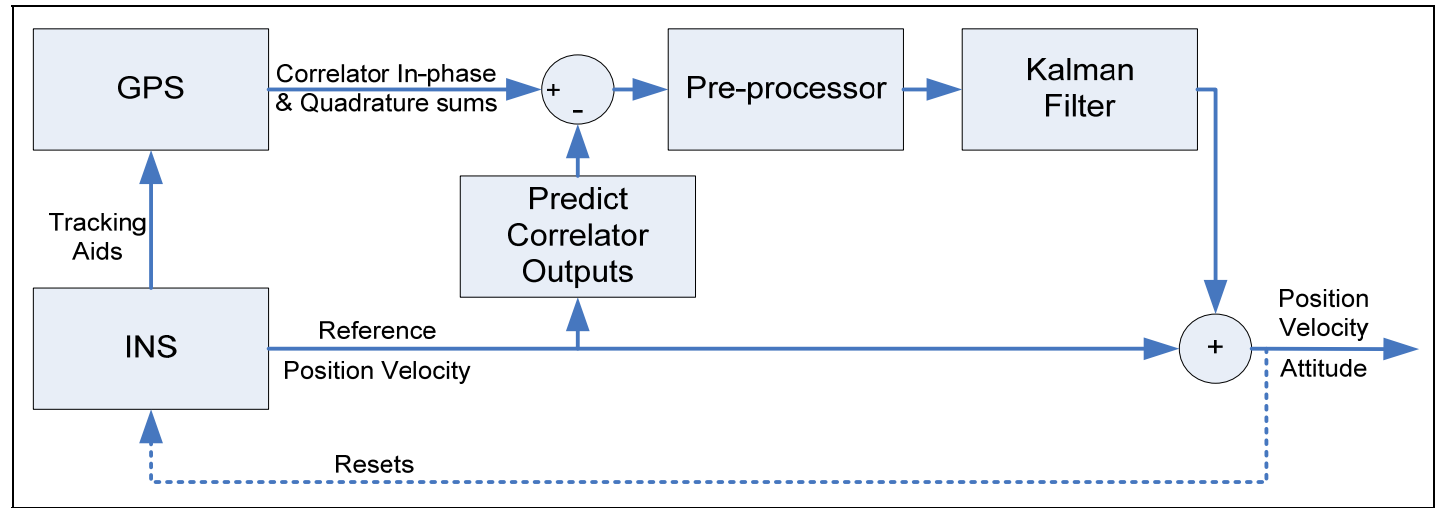

Figure 8 Flowchart of ultra tightly coupled integration 
This architecture can improve acquisition time and tracking performance of the GPS signals. The most important profit of this method is its ability to operate at lower signal to noise ratio levels. In this architecture, in-phase and quadrature samples of the GPS correlators can be used as measurement for the INS/GPS estimator [26].

In the context of this thesis study, a tightly-coupled INS/GPS integration architecture is utilized due to its higher performance than loosely coupled integration and relative simplicity compared to ultra-tightly coupled integration.

\subsection{IMU/GPS Specifications}

In the context of this thesis, a MEMS accelerometer and a MEMS gyroscope based low performance IMU is chosen as the basic component of the navigation system. The utilized MEMS IMU, MTi is a commercial off-the-shelf stabilization grade instrument produced by the Xsens Technologies Company. The performance specifications of this unit are gathered from its datasheet issued by the company and given in the following table.

Table 3 Specifications of the MEMS IMU

\begin{tabular}{|l|c|c|c|c|}
\hline Specifications & \multicolumn{2}{|c|}{ Gyroscope } & \multicolumn{2}{c|}{ Accelerometer } \\
\hline Bias repeatability & 200 & $\mathrm{deg} / \mathrm{m}$ & 40 & $\mathrm{mg}$ \\
\hline Scale factor repeatability & 1000 & $p p m$ & 1000 & $\mathrm{ppm}$ \\
\hline Misalignment & 0.1 & $\mathrm{deg}$ & 0.1 & $\mathrm{deg}$ \\
\hline Noise & 0.05 & $\mathrm{deg} / \mathrm{h} / \sqrt{\mathrm{hz}}$ & 0.002 & $\mathrm{~m} / \mathrm{s}^{2} / \sqrt{\mathrm{hz}}$ \\
\hline
\end{tabular}

In order to obtain realistic results from the simulations, aiding measurement system is also modeled using specifications of a commercial off-the-shelf GPS receiver. In the process of forming GPS measurements, reference pseudo range and pseudo range rate observables are calculated using reference satellite positions, velocities and reference user position, velocity. Zero mean Gaussian random noise is added to the reference pseudo range and pseudo range rate measurements to obtain realistic GPS measurements. Standard deviation parameters of the Gaussian distributions are 
gathered from commercial off-the-shelf GPS receiver's common specifications and given in Table 4.

Table 4 Specifications of the GPS receiver observables

\begin{tabular}{|l|l|}
\hline Pseudo range error $1 \sigma$ & $10 \mathrm{~m}$ \\
\hline Pseudo range rate error $1 \sigma$ & $0.1 \mathrm{~m} / \mathrm{s}$ \\
\hline
\end{tabular}

The reference satellite positions and velocities are acquired by using the satellite navigation toolbox of Matlab. Time specific data message (almanac) of GPS satellites is gathered from the official website of NASA [27]. The time specific data message is interpreted by the help of the toolbox and required information is recorded. Throughout the INS/GPS integration simulation, recorded satellite position and velocity data are used in an offline fashion.

\subsection{System Model}

An error state Kalman filter in a closed loop configuration is utilized for the INS/GPS integration process due to its lower processor load and simplicity [25]. The estimated error states are fed back at each time step to the nominal trajectory formed by processing inertial measurements and error states are reset after this feedback operation.

In the context of this setup, a state space which is composed of fifteen elements, namely three dimensional position, velocity and attitude errors, accelerometer biases and gyroscope biases, is exploited. The error state system model is obtained by linearizing the nonlinear navigation mechanization equations about the most recent navigation solution as given in Section 2.2.5. The following equation defines the system model for the designed estimators which can be written in the standard form as $\dot{X}=F X+G v$. 


$$
\left(\begin{array}{c}
\delta \dot{P} \\
\delta \dot{V} \\
\delta \dot{\psi} \\
\delta \dot{a}_{\text {Bias }} \\
\delta \dot{\omega}_{\text {Bias }}
\end{array}\right)=\left(\begin{array}{ccccc}
F_{P P} & F_{P V} & F_{P \psi} & 0 & 0 \\
F_{V P} & F_{V V} & F_{V \psi} & C_{B}^{N} & 0 \\
F_{\psi P} & F_{\psi V} & F_{\psi \psi} & 0 & C_{B}^{N} \\
0 & 0 & 0 & 0 & 0 \\
0 & 0 & 0 & 0 & 0
\end{array}\right)\left(\begin{array}{c}
\delta P \\
\delta V \\
\delta \psi \\
\delta a_{\text {Bias }} \\
\delta \omega_{\text {Bias }}
\end{array}\right)+\left(\begin{array}{cccc}
0 & 0 & 0 & 0 \\
C_{B}^{N} & 0 & 0 & 0 \\
0 & C_{B}^{N} & 0 & 0 \\
0 & 0 & I & 0 \\
0 & 0 & 0 & I
\end{array}\right)\left(\begin{array}{c}
v_{a} \\
v_{g} \\
v_{a b} \\
v_{g b}
\end{array}\right)
$$

Where $v_{a} \sim N\left(0, \sigma_{v_{a}}^{2}\right), v_{g} \sim N\left(0, \sigma_{v_{g}}^{2}\right)$ are accelerometer and gyroscope noise parameters respectively. $v_{a b} \sim N\left(0, \sigma_{v_{a b}}^{2}\right), v_{g b} \sim N\left(0, \sigma_{v_{g b}}^{2}\right)$ are accelerometer bias and gyroscope bias noise parameters respectively.

The entries of the state transition matrix are given by equations from (2.33) to (2.41) in Section 2.2.5.4.

\subsection{Discrete Time Equivalent System Model}

Inertial navigation is a discrete-time process since the analog outputs of the inertial sensors are converted to digital form by sampling at discrete time steps. Also the measurements from the GPS are acquired at discrete time points. Because of this nature of the navigation systems, discrete time difference equations for the system model and measurement model should be obtained instead of continuous-time differential equations as in the following format [28].

$$
\begin{gathered}
\dot{X}=F X+G w \\
X_{k+1}=\Phi_{k} X_{k}+w_{k}
\end{gathered}
$$

Where $X_{k}$ is the state vector at time step k and $\Phi_{k}$ is the state transition matrix.

The solution of the continuous time differential equation (4.2) can be denoted by the equation (4.3) and obtained as follows [29].

$$
X_{k+1}=e^{F\left(t_{k+1}-t_{k}\right)} X_{k}+\int_{t_{k}}^{t_{k+1}} e^{F\left(t_{k+1}-t_{k}-\tau\right)} G w(\tau) d \tau
$$

Where $\Delta t=t_{k+1}-t_{k}$ is the discrete time step. 
Assuming that the system dynamics matrix $\mathrm{F}$ is constant over a time period of $\Delta t$, the state transition matrix can be simply obtained by matrix exponential of $F \Delta t$ [28].

$$
\Phi_{k}=e^{F \Delta t}=I+F \Delta t+\frac{(F \Delta t)^{2}}{2 !}+\cdots
$$

The numerical evaluation of the discrete-time equivalent of process noise is more difficult than the state transition matrix. The following method proposes a simplified algorithm for the evaluation of the discrete-time process noise [28]. In this method, the matrix exponential of the matrix $\mathrm{A}$ is evaluated at first.

$$
\begin{gathered}
A=\left(\begin{array}{cc}
-F & G W G^{T} \\
0 & F^{T}
\end{array}\right) \\
B=\left(\begin{array}{cc}
\cdots & \Phi^{-1} Q_{k} \\
0 & \Phi^{T}
\end{array}\right)=e^{A} \\
Q_{k}=\Phi^{*} \Phi^{-1} Q_{k}
\end{gathered}
$$

Where $W$ is the power spectral density matrix of the process noise and $G$ is the scaling matrix of the process noise defined in Equation (4.2).

Then, by using the upper right and lower right parts of the matrix B, discrete-time equivalent process noise matrix $Q_{k}$ is obtained.

\subsection{Measurement Model}

The nonlinear measurement functions relating the state space variables and GPS measurements, namely pseudo range and pseudo range rates are given again in the equations (4.8) and (4.9) respectively for the sake of completeness.

$$
\begin{gathered}
h_{1}(X, Y, Z)=\sqrt{\left(X_{i}-X\right)^{2}+\left(Y_{i}-Y\right)^{2}+\left(Z_{i}-Z\right)^{2}} \\
h_{2}\left(V_{X}, V_{Y}, V_{Z}\right)=\sqrt{\left(V_{X_{i}}-V_{X}\right)^{2}+\left(V_{Y_{i}}-V_{Y}\right)^{2}+\left(V_{Z_{i}}-V_{Z}\right)^{2}}
\end{gathered}
$$


Where $\left(X_{i}, Y_{i}, Z_{i}\right)$ and $\left(V_{X_{i}}, V_{Y_{i}}, V_{Z_{i}}\right)$ correspond to ECEF position and velocity of the $i^{\text {th }}$ satellite. $(X, Y, Z)$ and $\left(V_{X}, V_{Y}, V_{Z}\right)$ correspond to the estimated user ECEF position and velocity.

However, a linear measurement model is required in the measurement update step of the extended Kalman filter for calculating the Kalman gain. The nonlinear measurement equations given are linearized with respect to the state space variables as given in Section 3.2.1.

Attitude error states and inertial sensor error states have negligible effects on the measurement innovation. Therefore the partial derivatives of the nonlinear measurement function with respect to these state variables are approximated as zero [9]. The following linearized measurement model is obtained as a function of partial derivatives of the measurement functions with respect to the positions and velocities.

$$
Y_{k+1}=H_{k} X_{k+1}
$$

Where $\left(X_{k}\right)$ corresponds to the state vector of the estimator.

$$
H_{k}=\left(\begin{array}{ccccc}
h_{1}^{T} & 0_{1 \times 3} & 0_{1 \times 3} & 0_{1 \times 3} & 0_{1 \times 3} \\
\vdots & \vdots & \vdots & \vdots & \vdots \\
h_{i}^{T} & 0_{1 \times 3} & 0_{1 \times 3} & 0_{1 \times 3} & 0_{1 \times 3} \\
0_{1 \times 3} & u_{1}^{T} & 0_{1 \times 3} & 0_{1 \times 3} & 0_{1 \times 3} \\
\vdots & \vdots & \vdots & \vdots & \vdots \\
0_{1 \times 3} & u_{i}^{T} & 0_{1 \times 3} & 0_{1 \times 3} & 0_{1 \times 3}
\end{array}\right)
$$

Where $\left(u_{i}^{T}\right)$ is the transpose of the unit vector pointing from the user to the $\mathrm{i}^{\text {th }}$ satellite defined in the $\mathrm{N}$-frame. North, east and down components of this unit vector for each satellite are represented as $\left(u_{i}^{N}, u_{i}^{E}, u_{i}^{D}\right)$ respectively. $\left(h_{i}^{T}\right)$ Component of the measurement matrix can be calculated as follows [9].

$$
h_{i}^{T}=\left[\begin{array}{lll}
\left(R_{N}+h\right) u_{i}^{N} & \left(R_{E}+h\right) u_{i}^{E} & -u_{i}^{D}
\end{array}\right]
$$




\section{CHAPTER 5}

\section{ESTIMATION TECHNIQUES}

This chapter of the thesis provides background information about the nonlinear estimation algorithms used in the INS/GPS integration process. In the first section, a short review of the Kalman Filtering is given with the mathematical models involved. In section 5.2 and 5.3, the Extended and Unscented Kalman Filters are discussed in detail, respectively. The adaptive scheme utilized for the Unscented Kalman Filter is described in the last section.

\subsection{The Kalman Filter}

The Kalman Filter (KF) is an optimal, recursive, minimum mean-square error estimator which can be utilized for the estimation of finite dimensional linear dynamic systems [30]. Discrete-time system and measurement models of the linear dynamic systems can be expressed in the following form.

$$
\begin{gathered}
x_{k+1}=F_{k} x_{k}+w_{k} \\
y_{k}=H_{k} x_{k}+v_{k}
\end{gathered}
$$

Where $x_{k}$ is the state vector, $F_{k}$ is the state transition matrix, and $w_{k}$ is the process noise matrix. $y_{k}$ is the measurement vector, $H_{k}$ is the measurement matrix and $v_{k}$ is the measurement noise vector. The equation (5.1) represents the system model which defines the evolution of the state with time. System model is used in the time update 
step of the KF. Time update is the step where the state is predicted and expected measurements are formed. Equation (5.2) represents the measurement model which defines the observables in terms of the state variables. Measurement model is used in the measurement update step of the KF. Measurement update is the step where the predicted state is corrected using incoming measurements.

The KF uses the basic assumptions of $w_{k}$ and $v_{k}$ being independent, zero-mean, additive Gaussian noise processes. These zero mean Gaussian processes can be denoted by $w_{k} \sim N\left(0, Q_{k}\right)$ and $v_{k} \sim N\left(0, R_{k}\right)$ where $Q_{k}$ and $R_{k}$ are the covariance matrices of the process and the measurement noise respectively. The standard KF algorithm is summarized in Table 5 [29].

State Space Model

$$
\begin{aligned}
& x_{k+1}=F_{k} x_{k}+w_{k} \\
& y_{k}=H_{k} x_{k}+v_{k}
\end{aligned}
$$

Initialization Of The Filter

$$
\begin{aligned}
& \hat{x}_{0}=E\left(x_{0}\right) \\
& P_{0}=E\left(\left(x_{0}-\hat{x}_{0}\right)\left(x_{0}-\hat{x}_{0}\right)^{T}\right)
\end{aligned}
$$

For $\mathrm{k}=0$ :end

Time Update

$$
\begin{aligned}
& \hat{x}_{k+1 \mid k}=F_{k} \hat{x}_{k \mid k} \\
& P_{k+1 \mid k}=F_{k} P_{k \mid k} F_{k}^{T}+Q_{k}
\end{aligned}
$$

Kalman Gain

$$
K_{k+1}=P_{k+1 \mid k} H_{k}^{T}\left(H_{k} P_{k+1 \mid k} H_{k}^{T}+R_{k+1}\right)^{-1}
$$

Measurement Update

$$
\begin{aligned}
& \hat{x}_{k+1 \mid k+1}=\hat{x}_{k+1 \mid k}+K_{k+1}\left(y_{k+1}-H_{k} \hat{x}_{k+1 \mid k}\right) \\
& P_{k+1 \mid k+1}=\left(I-K_{k+1} H_{k}\right) P_{k+1 \mid k}
\end{aligned}
$$




\subsection{The Extended Kalman Filter}

Standard KF focuses on the estimation of linear dynamic systems. However by the constitution of linearization procedure, the use of the KF can be extended to estimation of nonlinear systems. The resulting filter is referred as the Extended Kalman Filter [30]. The nonlinear system model and measurement model can be defined as in equations (5.8) and (5.9) respectively.

$$
\begin{gathered}
x_{k+1}=f_{k}\left(x_{k}\right)+w_{k} \\
y_{k}=h_{k}\left(x_{k}\right)+v_{k}
\end{gathered}
$$

Where $x_{k}$ is the state vector, $f_{k}\left(x_{k}\right)$ is the nonlinear state transition function, and $w_{k}$ is the process noise matrix. $y_{k}$ is the measurement vector, $h_{k}\left(x_{k}\right)$ is the nonlinear measurement function and $v_{k}$ is the measurement noise matrix. The basic assumption of $w_{k}$ and $v_{k}$ being independent, zero-mean, additive Gaussian noise processes also holds for the EKF.

The EKF uses the basic idea of linearizing the nonlinear functions $f_{k}\left(x_{k}\right)$ and $h_{k}\left(x_{k}\right)$ around the most recent state estimate $\hat{x}_{k \mid k}$ at each time step to calculate the related covariance matrices. The most vital drawback of the EKF is the linearization step of the nonlinear functions by calculating the Jacobian matrices as given in equation (5.10).

$$
\begin{aligned}
& F_{k}=\left.\frac{\partial f_{k}\left(x_{k}\right)}{\partial x}\right|_{x=x_{k}} \\
& H_{k}=\left.\frac{\partial h_{k}\left(x_{k}\right)}{\partial x}\right|_{x=x_{k}}
\end{aligned}
$$

Linearization procedure involved in the EKF algorithm by using first order Taylor series expansion can lead to second order errors in the mean and covariance of the state estimate depending on the order of nonlinearity of the system [31]. 
After obtaining the linearized system and measurement models, standard KF equations can be applied to the nonlinear system as given in Table 6 [30].

State Space Model

$$
\begin{aligned}
& x_{k+1}=f_{k}\left(x_{k}\right)+w_{k} \\
& y_{k}=h_{k}\left(x_{k}\right)+v_{k}
\end{aligned}
$$

Initialization Of The Filter

$$
\begin{aligned}
& \hat{x}_{0}=E\left(x_{0}\right) \\
& P_{0}=E\left(\left(x_{0}-\hat{x}_{0}\right)\left(x_{0}-\hat{x}_{0}\right)^{T}\right)
\end{aligned}
$$

For $\mathrm{k}=0$ :end

Calculate Jacobians

$$
\begin{aligned}
& F_{k}=\left.\frac{\partial f_{k}\left(x_{k}\right)}{\partial x}\right|_{x=x_{k}} \\
& H_{k}=\left.\frac{\partial h_{k}\left(x_{k}\right)}{\partial x}\right|_{x=x_{k}}
\end{aligned}
$$

Time Update

$$
\begin{aligned}
& \hat{x}_{k+1 \mid k}=f_{k}\left(\hat{x}_{k \mid k}\right) \\
& P_{k+1 \mid k}=F_{k} P_{k \mid k} F_{k}^{T}+Q_{k}
\end{aligned}
$$

Kalman Gain

$$
K_{k+1}=P_{k+1 \mid k} H_{k}^{T}\left(H_{k} P_{k+1 \mid k} H_{k}^{T}+R_{k+1}\right)^{-1}
$$

Measurement Update

$$
\begin{aligned}
& \hat{x}_{k+1 \mid k+1}=\hat{x}_{k+1 \mid k}+K_{k+1}\left(y_{k+1}-h_{k}\left(\hat{x}_{k+1 \mid k}\right)\right) \\
& P_{k+1 \mid k+1}=\left(I-K_{k+1} H_{k}\right) P_{k+1 \mid k}
\end{aligned}
$$




\subsection{The Unscented Kalman Filter}

The state distribution is approximated as a Gaussian random variable in the EKF implementation. Mean and covariance matrix of the state vector are propagated through the first order linearization of the nonlinear system model. Large linearization errors can be introduced in the mean and covariance of posterior distribution leading to suboptimal performance and even divergence of the filter [29].

Another nonlinear estimator, Unscented Kalman Filter (UKF) approaches this problem by carefully selecting deterministic sigma points from the Gaussian distribution completely capturing mean vector and covariance matrix. These points are propagated through the nonlinear function itself leading to third order errors for any nonlinearity. Therefore, the assumption of state being Gaussian distributed holds for both the EKF and UKF, but representation of this distribution by the help of deterministic sigma points instead of mean and covariance matrix makes the UKF superior in performance [32] .

The basic operation of the UKF is Unscented Transformation (UT) which is a method of calculating the statistics of a random variable undergoing a nonlinear transformation [30]. The sigma points are determined by the help of UT. In the literature, there are various methods utilized for the UT. The Scaled Unscented Transformation (SUT) is one of the sigma point selection methods which gives the opportunity to adjust the spread of sigma points and control the higher order errors by some design parameters [33].

The algorithm for the SUT is given in Table 7 [33]. There are three design parameters in the given algorithm. The parameter $\alpha$ determines the spread of sigma points around the given state. It is usually set to a small value. The parameter $\beta$ is used to incorporate the prior knowledge about the distribution of the state space variables. The optimal value of this parameter for Gaussian distributions is asserted as 2 . The parameter $\kappa$, which is a secondary constant controlling higher order effects, is usually set to 0 . In the literature, there is not a compromised method for the 
selection of these parameters. Therefore, determination of these parameters is problem specific [34].

Select Sigma Points

$$
\begin{aligned}
& X_{0}=\hat{x}_{k \mid k} \\
& X_{i}=\hat{x}_{k \mid k}+\left((N+\lambda) P_{k \mid k}\right)_{i}^{1 / 2}, i=1, \ldots, N \\
& X_{i}=\hat{x}_{k \mid k}-\left((N+\lambda) P_{k \mid k}\right)_{i}^{1 / 2}, i=N+1, \ldots, 2 N
\end{aligned}
$$

Weights of Sigma Points

$$
\begin{aligned}
& W_{0}^{(m)}=\frac{\lambda}{N+\lambda} \\
& W_{0}^{(c)}=\frac{\lambda}{N+\lambda}+\left(1-\alpha^{2}+\beta\right) \\
& W_{i}^{(m)}=W_{i}^{(c)}=\frac{1}{2(N+\lambda)}, i=1, \ldots, 2 N
\end{aligned}
$$

The Parameters Of The Scaled Unscented Transformation

$$
\begin{aligned}
& \lambda=\alpha^{2}(N+\kappa)-N \\
& N: \text { Dimension of the state space } \\
& \alpha: 10^{-4} \leq \alpha \leq 1 \text {, Usually set to a small positive value } \\
& \kappa: \kappa=3-N, \text { A secondary scaling parameter } \\
& \beta: \beta=2 \text {, Optimal for Gaussian distributions }
\end{aligned}
$$

According to the SUT method given above, $2 \mathrm{~N}+1$ sigma points are generated from the Gaussian distribution where $\mathrm{N}$ is the dimension of the state space [35]. The UKF makes use of these sigma points by propagating them through the nonlinear system and measurement models [36]. A generic algorithm for the UKF is given in Table 8.

In the context of this study, an error state space model is utilized as the system model which is given in Equation (4.1). The system model of the INS/GPS integration filter is linear. Therefore, the time update step of the UKF is done using the state propagation routine of the KF. 
State Space Model

$$
\begin{aligned}
& x_{k+1}=f_{k}\left(x_{k}\right)+w_{k} \\
& y_{k}=h_{k}\left(x_{k}\right)+v_{k}
\end{aligned}
$$

Initialization of the filter

$$
\begin{aligned}
& \hat{x}_{0}=E\left(x_{0}\right) \\
& P_{0}=E\left(\left(x_{0}-\hat{x}_{0}\right)\left(x_{0}-\hat{x}_{0}\right)^{T}\right)
\end{aligned}
$$

For $\mathrm{k}=0$ :end

Calculate the sigma points

$$
X_{k}=\left(\begin{array}{lll}
\hat{x}_{k} & \hat{x}_{k}+\gamma \sqrt{P_{k}} & \hat{x}_{k}-\gamma \sqrt{P_{k}}
\end{array}\right)
$$

Time Update

$$
\begin{aligned}
& \hat{X}_{k+1 \mid k}=f_{k}\left(X_{k}\right) \\
& \hat{x}_{k+1 \mid k}=\sum_{i=0}^{2 N} W_{i}^{(m)} \hat{X}_{i, k+1 \mid k} \\
& P_{k+1 \mid k}=\sum_{i=0}^{2 N} W_{i}^{(c)}\left(\hat{X}_{i, k+1 \mid k}-\hat{x}_{k+1 \mid k}\right)\left(\hat{X}_{i, k+1 \mid k}-\hat{x}_{k+1 \mid k}\right)^{T}+Q_{k}
\end{aligned}
$$

Measurement Prediction

$$
\begin{aligned}
& Y_{k+1 \mid k}=h_{k}\left(\hat{X}_{k+1 \mid k}\right) \\
& \hat{y}_{k+1}=\sum_{i=0}^{2 N} W_{i}^{(m)} Y_{i, k+1 \mid k}
\end{aligned}
$$

Kalman Gain

$$
\begin{aligned}
& P_{y_{k+1} y_{k+1}}=\sum_{i=0}^{2 N} W_{i}^{(c)}\left(Y_{i, k+1 \mid k}-\hat{y}_{k+1}\right)\left(Y_{i, k+1 \mid k}-\hat{y}_{k+1}\right)^{T}+R_{k+1} \\
& P_{x_{k+1} y_{k+1}}=\sum_{i=0}^{2 N} W_{i}^{(c)}\left(\hat{X}_{i, k+1 \mid k}-\hat{x}_{k+1}\right)\left(Y_{i, k+1 \mid k}-\hat{y}_{k+1}\right)^{T} \\
& K_{k+1}=P_{x_{k+1} y_{k+1}} P_{y_{k+1} y_{k+1}}^{-1}
\end{aligned}
$$

Measurement Update

$$
\begin{aligned}
& \hat{x}_{k+1 \mid k+1}=\hat{x}_{k+1 \mid k}+K_{k+1}\left(y_{k+1}-\hat{y}_{k+1}\right) \\
& P_{k+1 \mid k+1}=K_{k+1} P_{y_{k+1} y_{k+1}} K_{k+1}^{T}
\end{aligned}
$$




\subsection{The Adaptive Unscented Kalman Filter}

As mentioned in Section 5.3, the underlying routine for the UKF is the UT which determines the sigma points representing the related Gaussian distributions. Since the SUT gives the opportunity to adjust the spread of sigma points and control the higher order errors by some design parameters, it is selected as the UT method in the context of this thesis.

When the effects of the SUT parameters on integrated navigation solution are investigated, it is observed that the performance of the UKF is dependent on these design parameters. Selection of different parameters for the same navigation scenario results in varying navigation accuracy [37]. Also, in-flight performance of the integration filter changes for different parts of the trajectory for a given set of SUT parameters.

The parameter $\alpha$, which is usually set to a small value between 0 and 1 , determines the spread of sigma points around the given state. The parameter $\beta$, whose optimal value for Gaussian distributions is 2 , is used to incorporate the prior knowledge about the distribution of the state space variables. The parameter $\kappa$, which is a secondary constant controlling higher order effects, is usually set to 0 .

Among these three parameters, $\alpha$ is the most influential one in affecting the UKF performance. When the parameters $\beta$ and $\kappa$ are set to their default values as 2 and 0 , respectively, different values of $\alpha$ changes the spread of sigma points explicitly.

In Table 9, the spread of the sigma points from the mean value of the state can be observed as a function of the standard deviation of the state estimate $(\sigma)$. Also related weights of the sigma points are given in the same table. 
Table 9 Spread of $\sigma$-points for different $\alpha$ parameters

\begin{tabular}{|c|c|c|c|}
\hline $\begin{array}{c}\text { Value of alpha } \\
(\alpha)\end{array}$ & $\begin{array}{c}\text { Distinction of } \\
\sigma \text {-points wrt.mean }\end{array}$ & $\begin{array}{c}\text { Weight } \\
W_{0}^{(m)}\end{array}$ & $\begin{array}{c}\text { Weight } \\
W_{0}^{(c)}\end{array}$ \\
\hline 0.1 & $0.39 \sigma$ & -98.99 & -96.01 \\
\hline 0.2 & $0.77 \sigma$ & -24.00 & -21.04 \\
\hline 0.3 & $1.16 \sigma$ & -10.11 & -7.20 \\
\hline 0.4 & $1.55 \sigma$ & -5.25 & -2.41 \\
\hline 0.5 & $1.94 \sigma$ & -3.00 & -0.25 \\
\hline
\end{tabular}

It can be inferred from Table 9 that, for small values of $\alpha$, selected sigma points are close to the mean and they have relatively large weights. When the parameter $\alpha$ is raised, spread of the sigma points are extended and they move away from the mean with reduced weights. Depending on the uncertainty of the state estimate, state covariance matrix grows or shrinks. For large state covariance matrices, meaning high uncertainties, a large value of the parameter $\alpha$ results in sigma points which are far away from the mean. It is observed from the simulations that; in the INS/GPS integration process, such a situation may result in divergence of the UKF. But when the uncertainties of the state estimate are low, better performance can be obtained with larger values of the parameter $\alpha$. When elements of the state covariance matrix are relatively small, a small value of the parameter $\alpha$ results in heavily weighted sigma points which are very close to the mean. This situation resembles to the EKF which uses just the mean value of the state in order to represent the state estimate.

Proceeding from this point of view, selection of a common value for the parameter $\alpha$ is not a reasonable choice for the systems whose uncertainty may change drastically in time which is the case for INS/GPS integrated systems. Since GPS is vulnerable to jamming and signal blockage, intermittence of this aiding system can occur frequently. When the GPS aiding is obtained regularly, covariance matrices and mean of the state estimate can be kept bounded. But when the GPS outage is considered, accuracy of the state estimate degrades with time depending on the duration of measurement outage. Therefore, it is proposed that, different values of 
the SUT parameters should be utilized for various uncertainties of the state estimate adaptively in order to obtain a better performance from the integration filter. Actually, this method turns out to be an optimal tuning strategy, results of which will be used adaptively during the operation of the INS/GPS integrated system. In order to justify the effectiveness of this approach, four different scenarios for the INS/GPS integration process are constituted as follows.

- Steady state conditions for the system when GPS is available

- End of 20 seconds of GPS outage

- End of 30 seconds of GPS outage

- End of 40 seconds of GPS outage

For each of these scenarios, Monte Carlo Analysis method is utilized. 13 values for the parameter $\alpha$ are specified in the range of 0.01 to 0.6 , and 1000 runs are set for each of these parameters. RMS errors for each individual run is calculated over time and mean of these runs are formed in order to compare the performance of the system with different $\alpha$ values. In this analysis, each individual run lasts for two measurement update periods. Because, covariance of the states converges so quickly that, all of the scenarios reach to the steady state in a few seconds. Main concern of this application is the transient response period of the scenarios. Therefore first two seconds after the GPS outage period are considered. Optimal values for the parameter $\alpha$ and RMSE improvement percentage of AUKF with respect to EKF integration for each individual scenario is given in Table 10 .

Table 10 Optimal Alpha values and RMSE improvement percentage

\begin{tabular}{|c|c|c|c|c|}
\hline \multirow{2}{*}{$\begin{array}{c}\text { GPS Outage } \\
\text { Period }(\mathrm{s})\end{array}$} & \multirow{2}{*}{$\begin{array}{c}\text { Optimal } \\
\text { Alpha }(\alpha)\end{array}$} & Position & Velocity & Attitude \\
\cline { 3 - 5 } & 0.40 & 3.3 & 6.3 & 1.9 \\
\hline 0 & 0.30 & 16.6 & 14.3 & 2.5 \\
\hline 20 & 0.25 & 22.3 & 24.5 & 2.2 \\
\hline 30 & 0.15 & 32.4 & 25.8 & 2.9 \\
\hline
\end{tabular}


It is observed from Table 10 that, for larger periods of GPS outage, meaning larger uncertainty in the state estimate, smaller $\alpha$ values give better performance in terms of RMSE. In the INS/GPS integration process, basic parameter that includes the effects of all sensor and modeling errors is the position state. Both of the velocity and attitude errors accumulate in this state. Therefore, submatrix of the state covariance matrix corresponding to the position states is an indicator for the total uncertainty of the navigation solution. In this analysis, determinant of the aforementioned submatrix is utilized as the generalized variance of the system. The generalized variance is evaluated in log scale and segments of the flight trajectory are denoted by intervals of this metric as given in Table 11.

Table 11 Generalized variance intervals for different GPS outage periods

\begin{tabular}{|c|c|c|c|c|}
\hline GPS Outage Period (seconds) & Steady State & 20 & 30 & 40 \\
\hline Generalized Variance $(\log |P|)$ & $8-13$ & $13-20$ & $20-27$ & $27-35$ \\
\hline Alpha & 0.4 & 0.3 & 0.25 & 0.15 \\
\hline
\end{tabular}

Throughout the flight trajectory, generalized variance metric is evaluated. Depending on this value, the SUT parameter alpha is designated using the mapping given in Table 11 adaptively. This method makes the use of optimal choice of the SUT parameters possible. The better choice of the parameters is the underlying reason for the improvement in the navigation system performance.

The variation of the RMSE index for different $\alpha$ values can be observed in Figure 9 to Figure 12 separately for each scenario. RMSE budget of the EKF, which is the basic estimation algorithm for the INS/GPS integration, is given at each scenario together with the standard deviations of UKF RMSE in order to allow a comparison of the performance indices. 


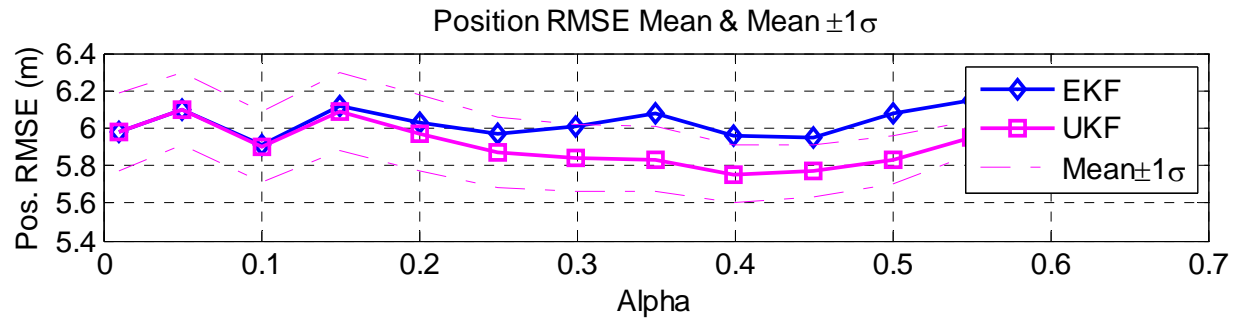

Velocity RMSE Mean \& Mean $\pm 1 \sigma$

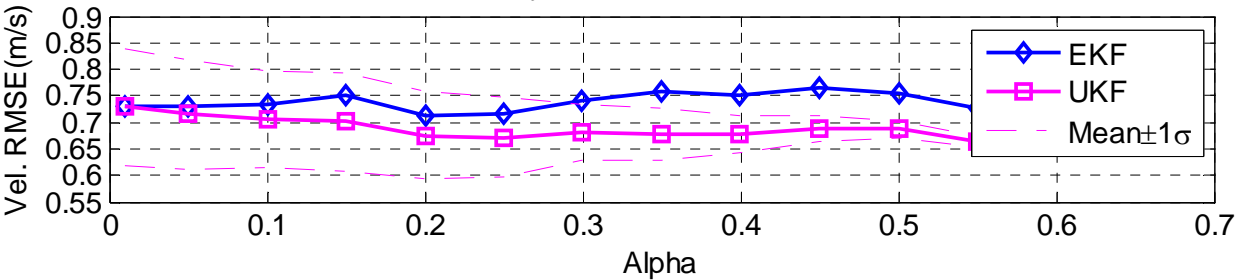

Attitude RMSE Mean \& Mean $\pm 1 \sigma$

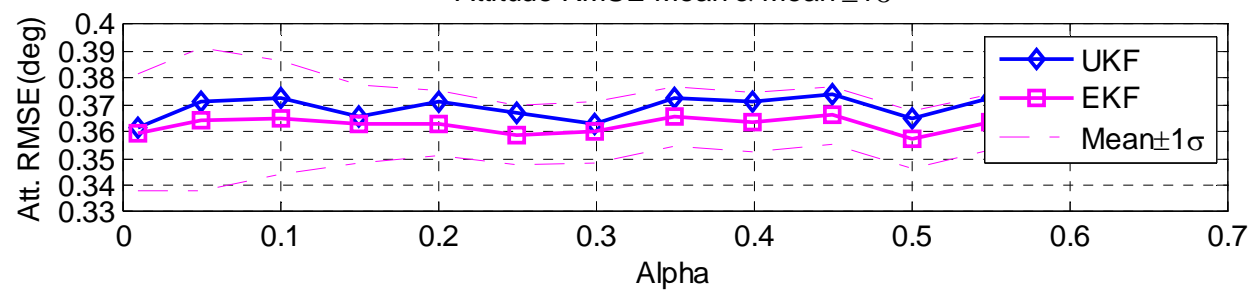

Figure 9 RMSE variations over alpha values for Steady state conditions

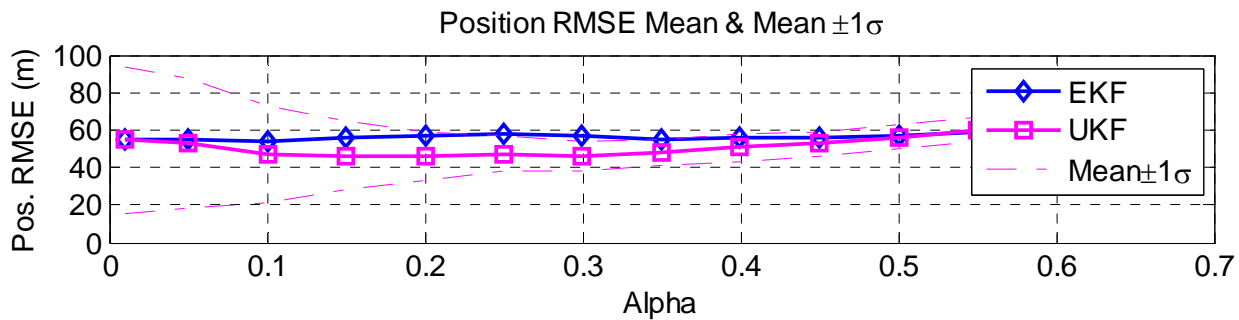

Velocity RMSE Mean \& Mean $\pm 1 \sigma$

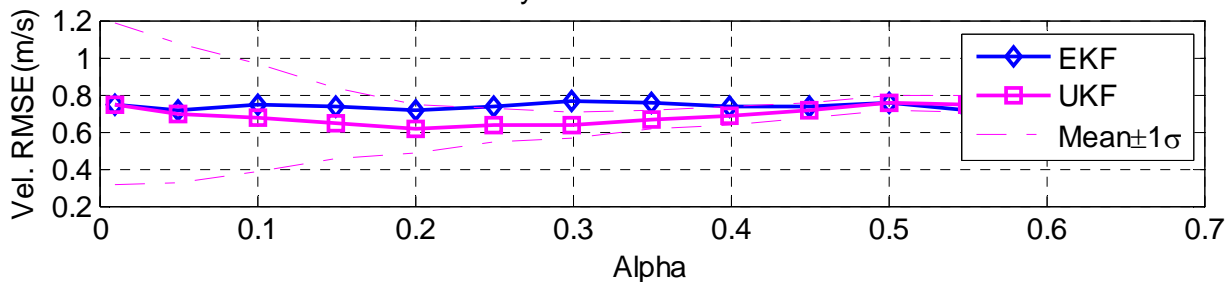

Attitude RMSE Mean \& Mean $\pm 1 \sigma$

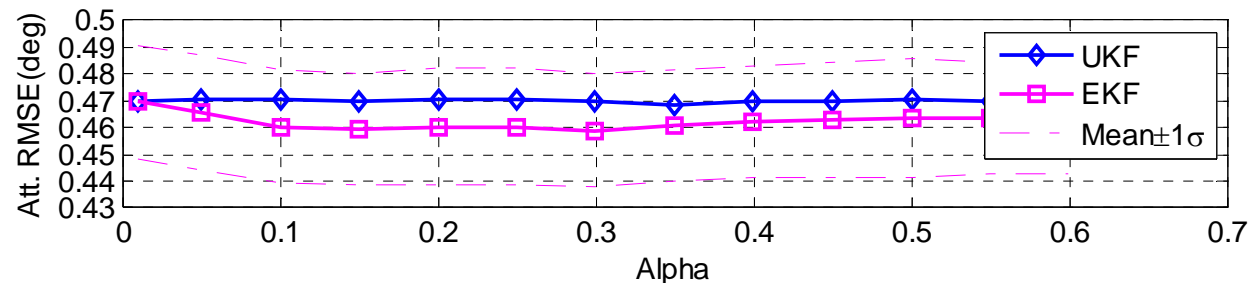

Figure 10 RMSE variations over alpha values for 20 seconds GPS Outage 


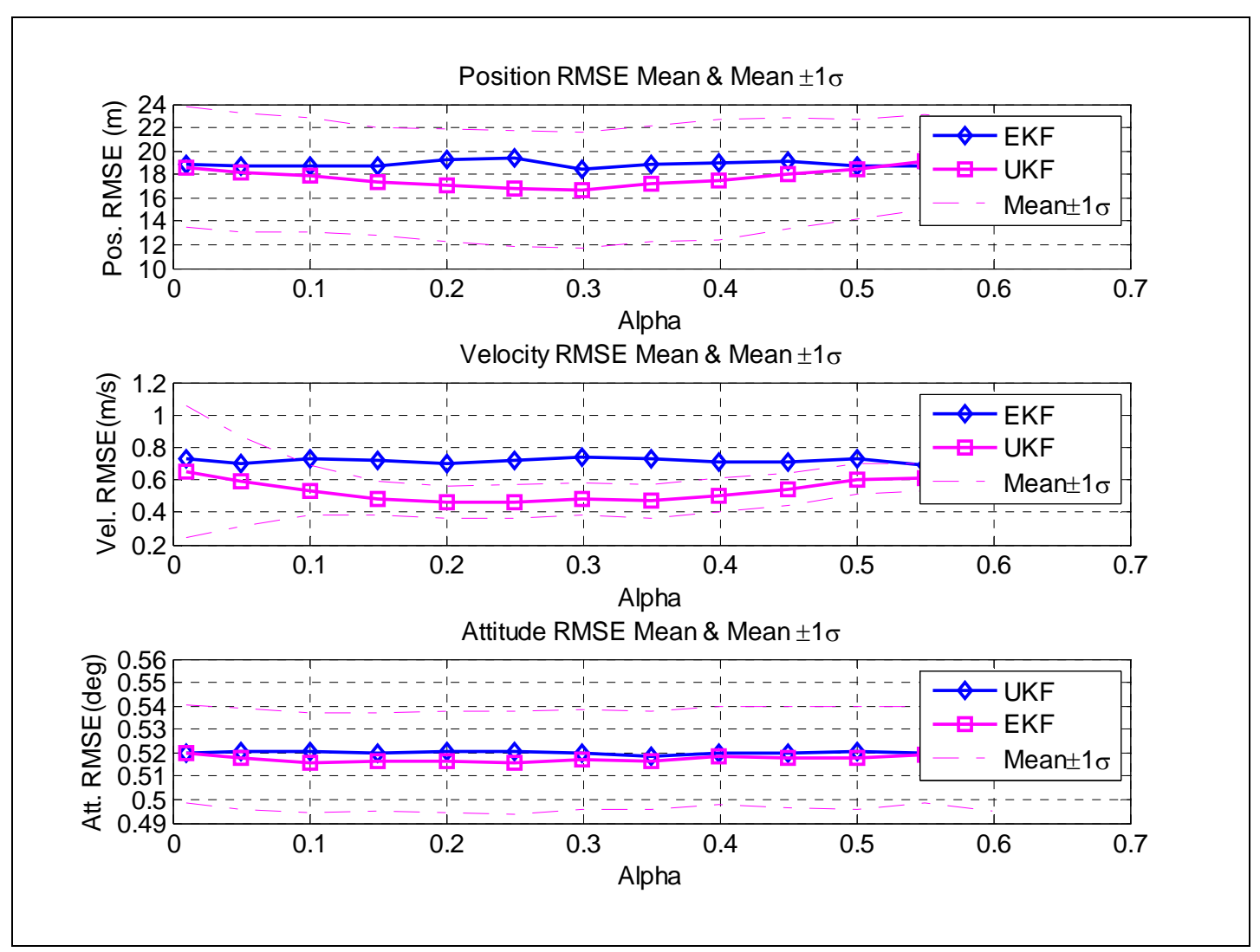

Figure 11 RMSE variations over alpha values for 30 seconds GPS Outage

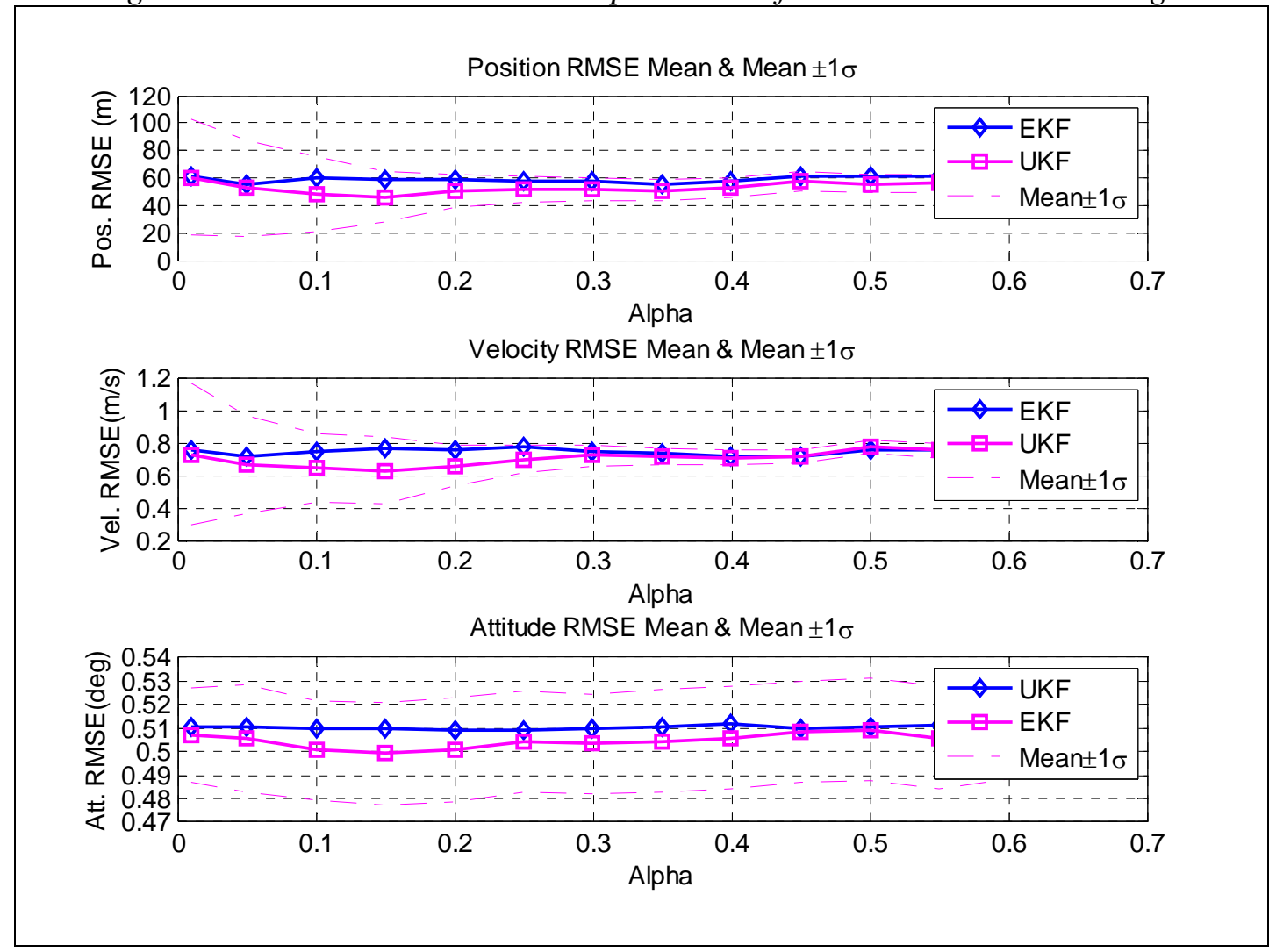

Figure 12 RMSE variations over alpha values for 40 seconds GPS Outage 


\section{CHAPTER 6}

\section{RESULTS AND DISCUSSIONS}

In this chapter, simulation and field test results of the INS/GPS integration process are given. In the first part, a realistic fighter flight trajectory, which is generated by the INS toolbox of Matlab is used as the main navigation scenario. This scenario is exploited to simulate IMU and GPS data within Monte Carlo analysis. Results of the proposed AUKF estimation method are compared with the standard EKF and UKF techniques. In the second part of the chapter, results of the field tests conducted using a tactical grade INS/GPS integrated system and a low performance MEMS IMU are examined.

\subsection{Simulation Results}

Simulation results are considered in three subsections as follows.

\subsubsection{Reference and Standalone Inertial Navigation Results}

The reference navigation solution of the flight trajectory is given in the following figures to visualize the generated scenario. Three dimensional flight trajectory can be observed in Figure 13 and Figure 14. The Figure 15 and Figure 16 show the linear acceleration and angular rate outputs of the simulated and reference IMU as a function of time respectively. Figure 17, Figure 18 and Figure 19 represent the ground truth and standalone INS navigation solutions namely the position, velocity and the attitude of the platform. The results of the accumulation of inertial sensor errors throughout a realistic flight trajectory can be observed in these figures. 


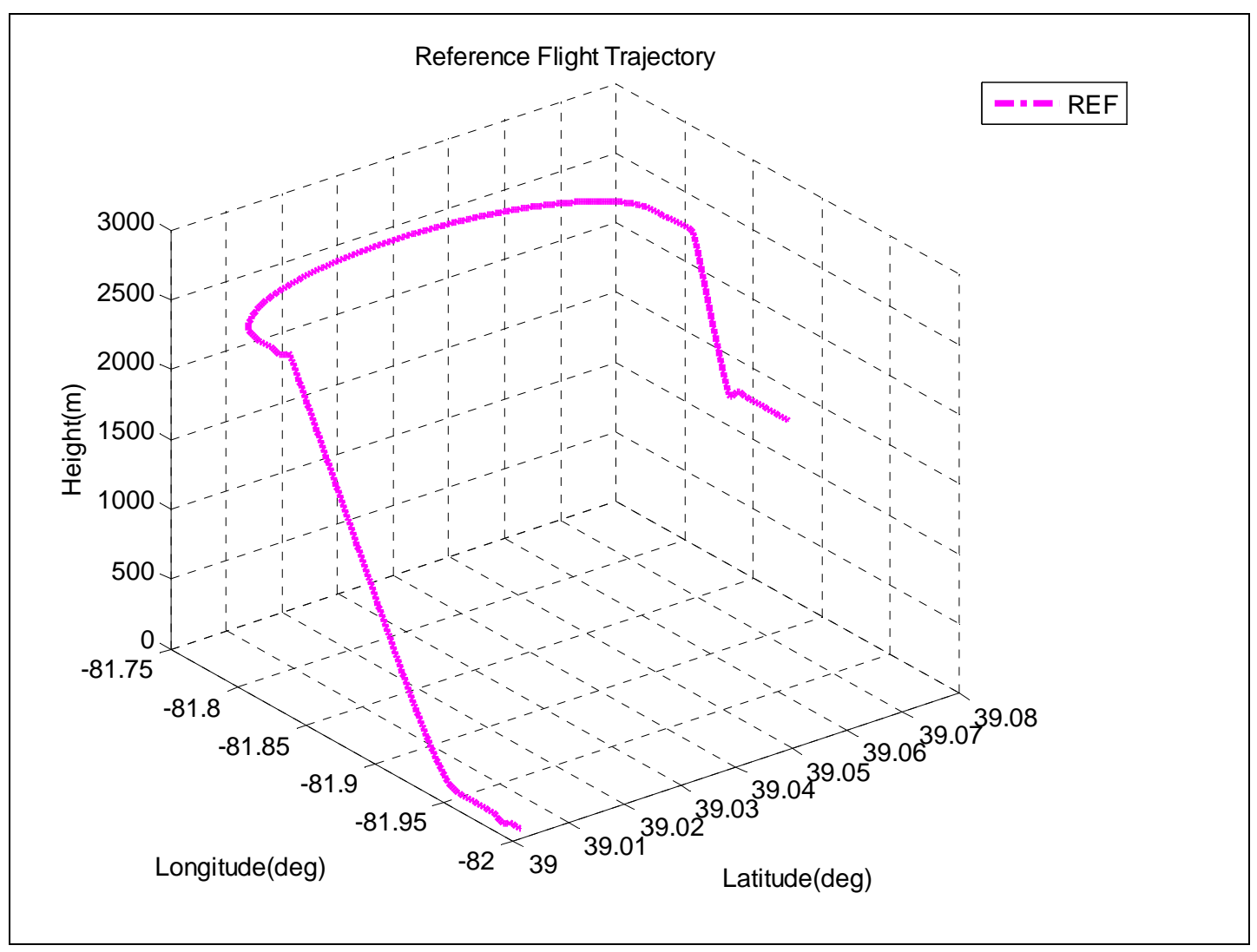

Figure 13 Reference flight trajectory in 3D space

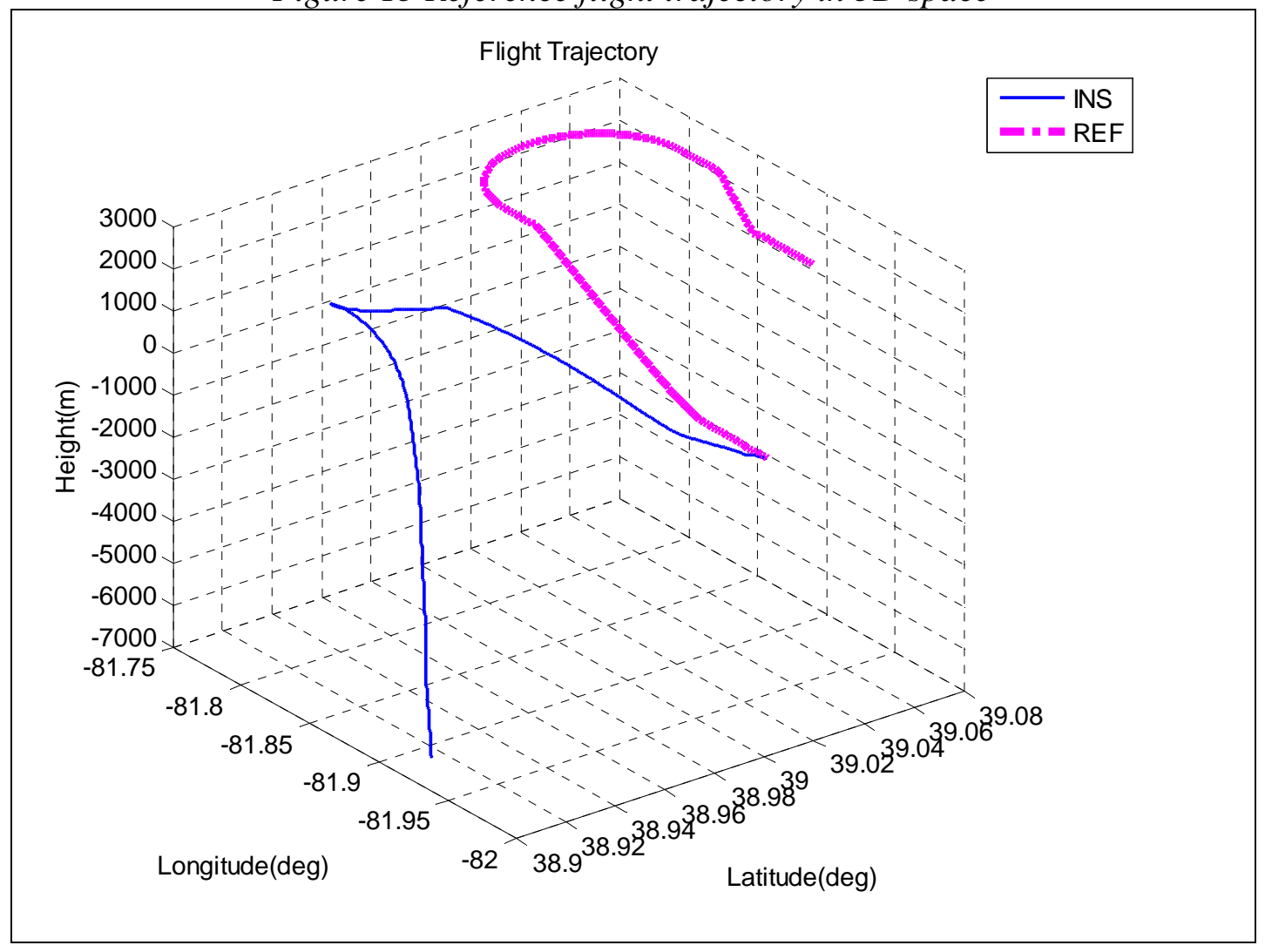

Figure 14 Standalone INS and reference flight trajectories in 3D space 


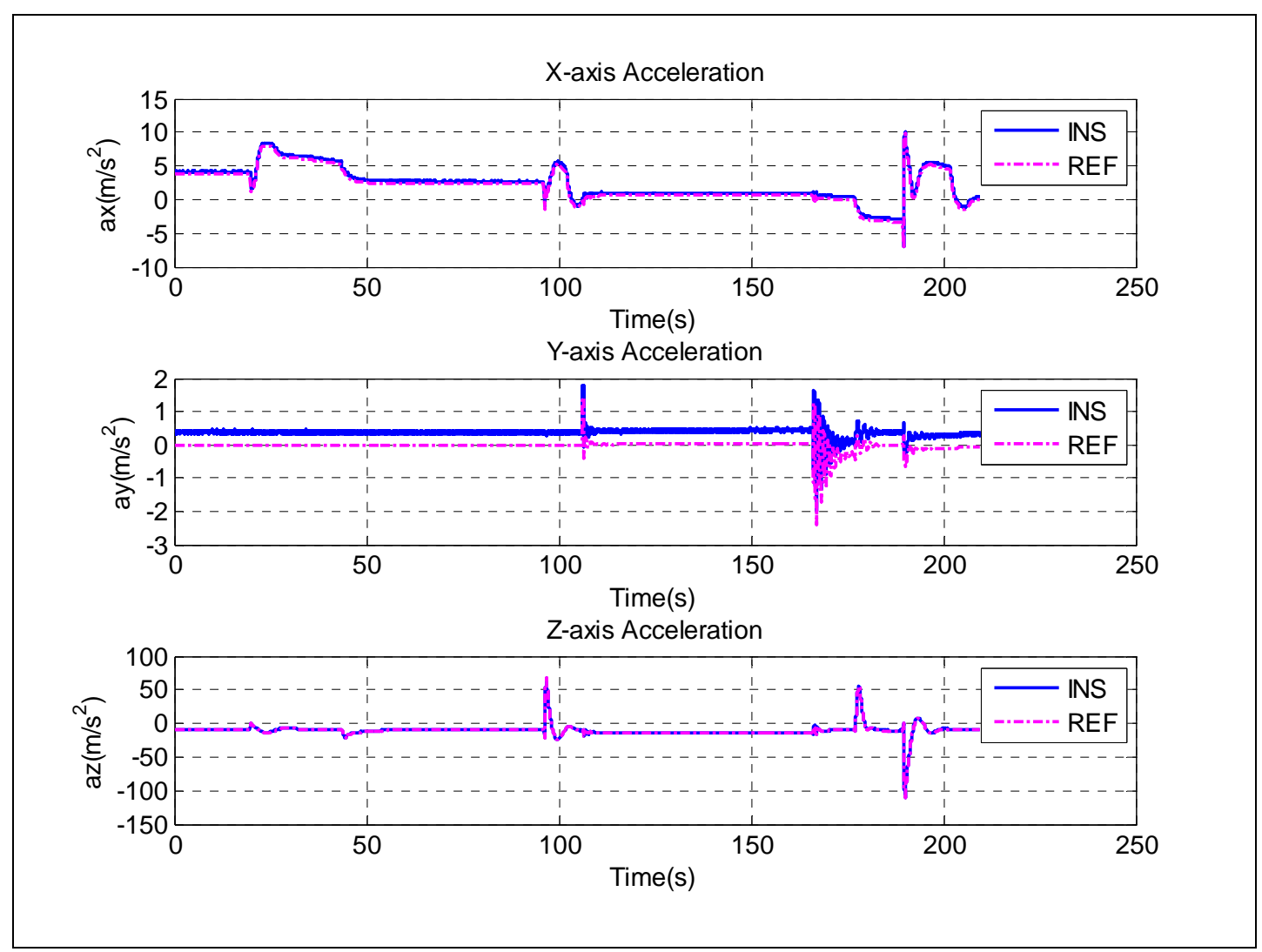

Figure 15 Simulated acceleration output of the IMU

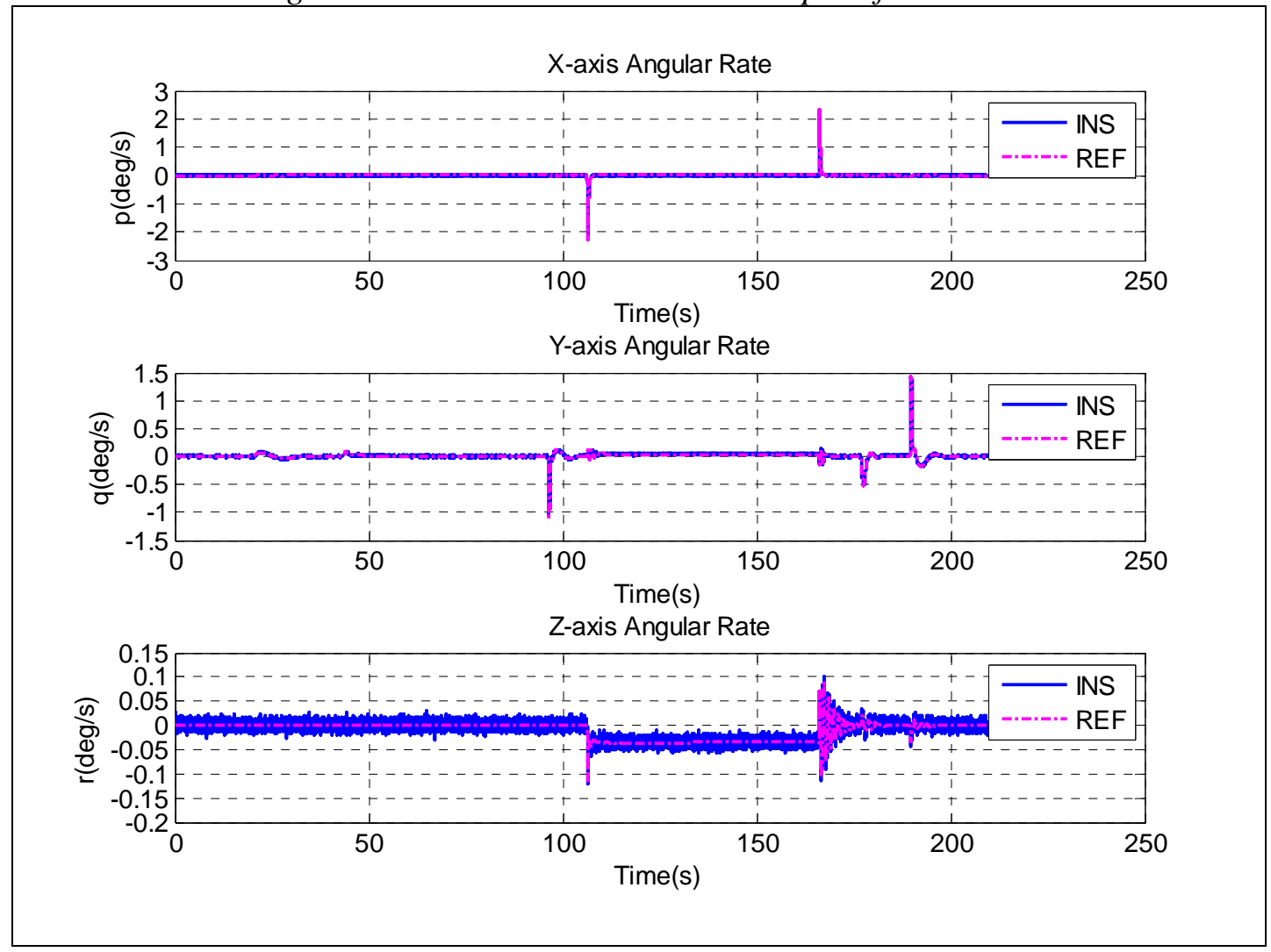

Figure 16 Simulated angular rate output of the IMU 


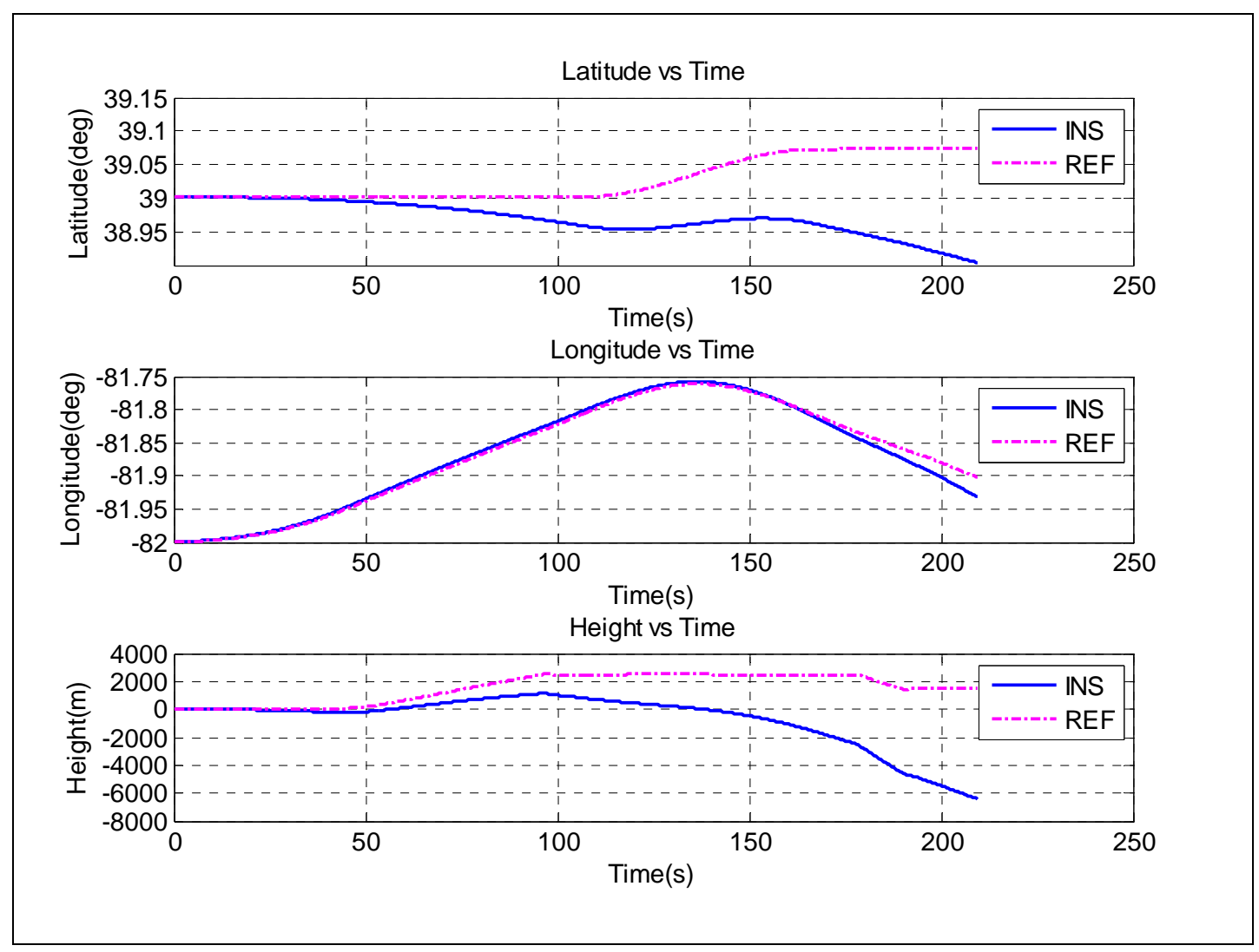

Figure 17 Position output of standalone INS

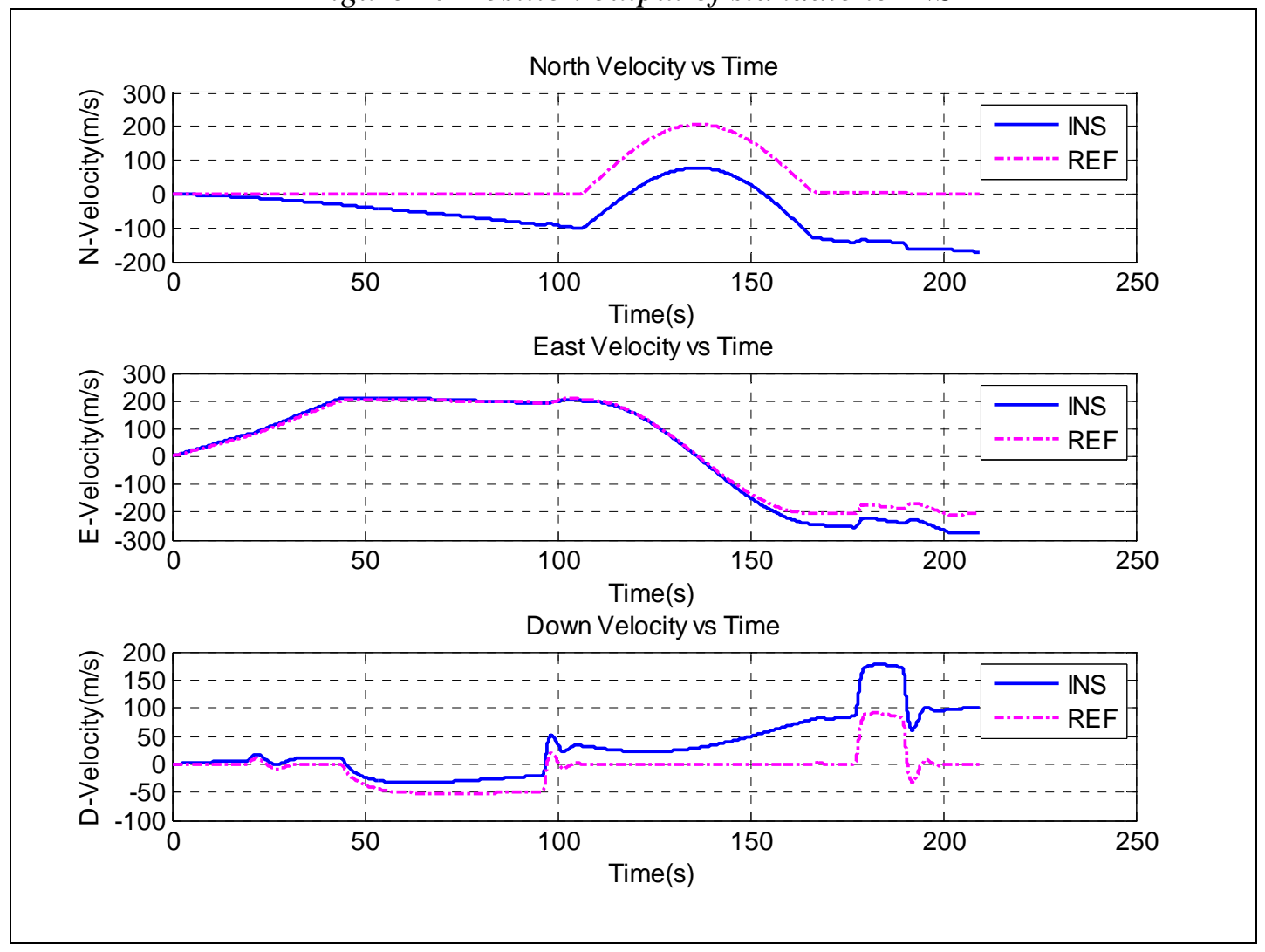

Figure 18 Velocity output of standalone INS 


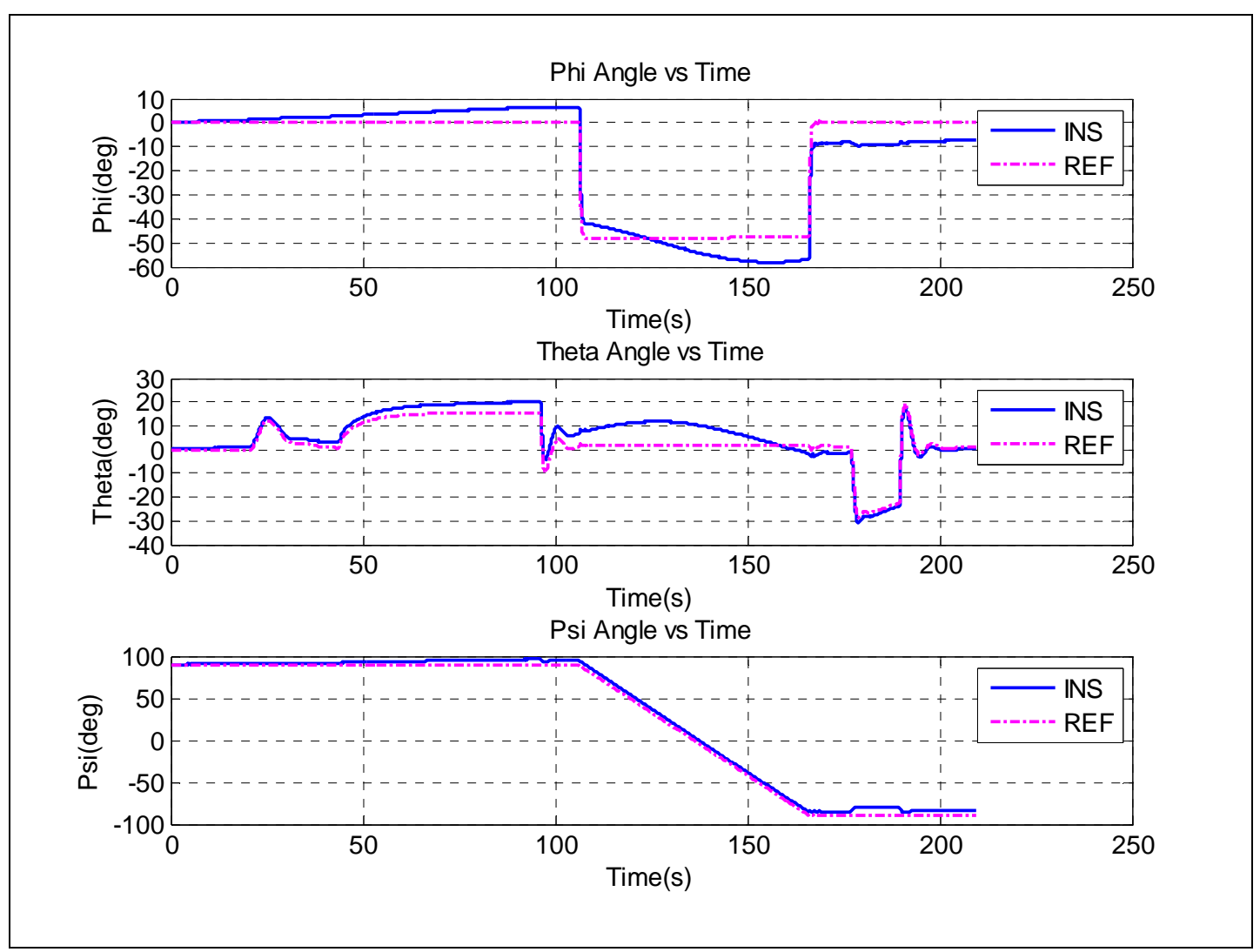

Figure 19 Attitude output of standalone INS

It is clear from Figure 14 that a low cost MEMS IMU can not be used as a sole navigation system after a few minutes from the take off. Accumulation of large errors present in the outputs of the low performance inertial sensors result in largescaled navigation errors which can not be tolerated for most of the moving platforms. This result makes integration of two complementary systems, namely INS and GPS essential.

\subsubsection{Standard EKF and UKF Results}

Low cost and low performance MEMS IMU is integrated with the GPS in a tightlycoupled architecture using the standard EKF and UKF algorithms. Identical system and measurement models are utilized in both of the filters. The same initial state covariance, process noise and measurement noise matrices are used in the estimation algorithms for a meaningful comparison of the performances. 
As mentioned in section 4.6, the nonlinearity of the INS/GPS integrated system stems from the utilization of the nonlinear measurement model. In the presence of the regular measurements, the estimation error of the navigation solution is kept bounded. Therefore, the nonlinearity in the measurements can easily be handled by the EKF.

When large initial state errors and covariances are considered, the first order approximation of the EKF becomes insufficient to cope with the nonlinearity. These situations may result in even divergence of the filter. Since GPS is vulnerable to jamming and signal blockage, outage of this aiding system should be considered carefully. Intermittence of the GPS measurements results in large initial state errors and large initial state covariances for the estimation algorithms depending on the period of standalone practice of the INS. In the course of the analysis, three GPS outage periods are modeled to create a compelling scenario for the estimation algorithms. Hence the actual performance of the EKF and the UKF are compared in a more compelling and realistic case. Simulated GPS outage periods are summarized in the following table.

Table 12 GPS outage periods in the flight trajectory

\begin{tabular}{|c|c|c|c|c|c|c|}
\hline GPS Availability & OFF & ON & OFF & ON & OFF & ON \\
\hline Time Period (s) & $0-20$ & $20-60$ & $60-90$ & $90-130$ & $130-170$ & $170-210$ \\
\hline
\end{tabular}

In Figure 20 to Figure 29, estimation errors for the curvilinear position, the velocity, the attitude and the sensor biases for a single scenario are presented together with the standard deviations of the two estimation algorithms consecutively. In Figure 30, the Root Mean Square Error values of the two filters throughout the trajectory are given in order to compare the performances. 

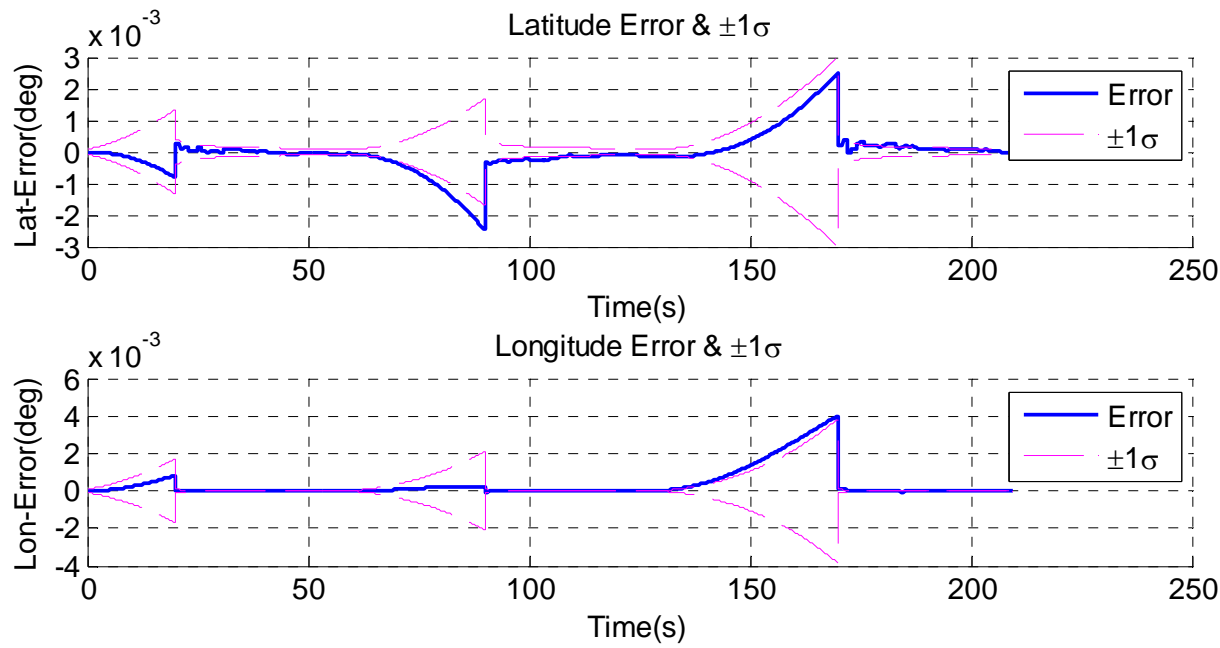

Height Error \& $\pm 1 \sigma$

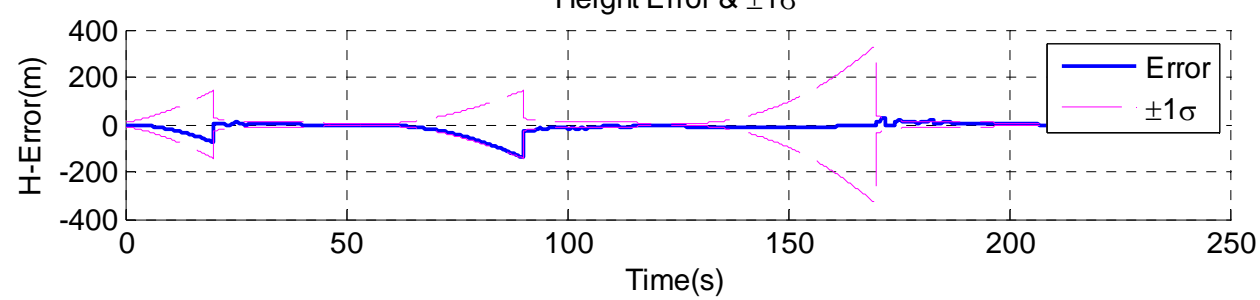

Figure 20 EKF Position estimation error and $\pm 1 \sigma$ error bound

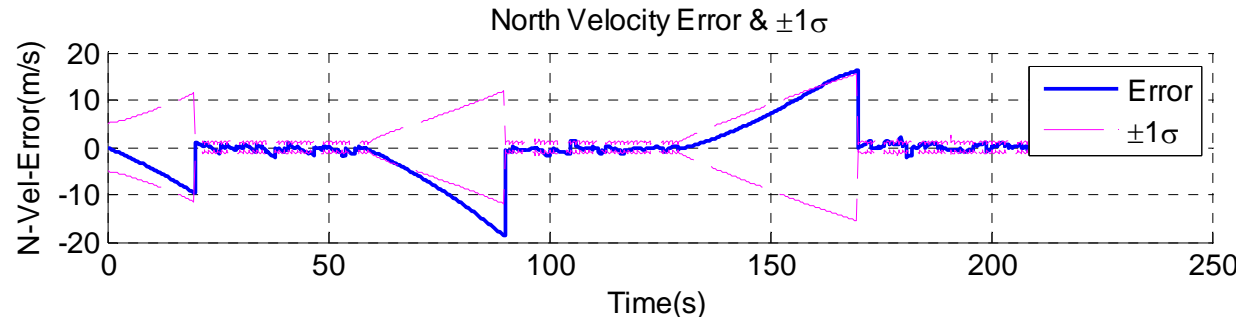

East Velocity Error $\& \pm 1 \sigma$

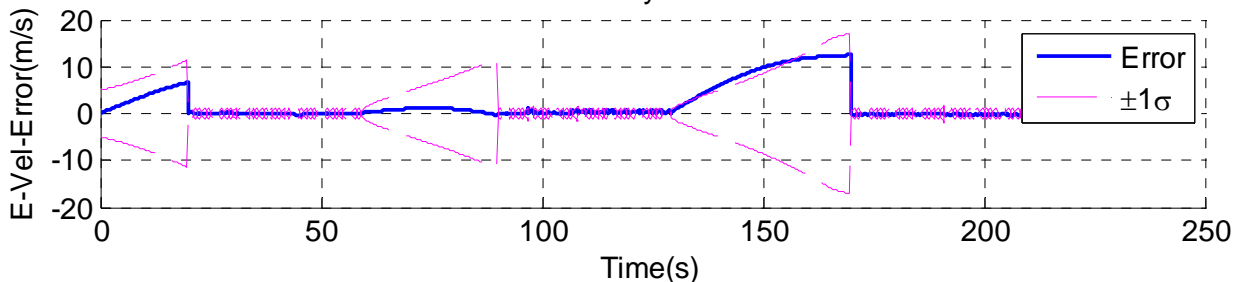

Down Velocity Error $\& \pm 1 \sigma$

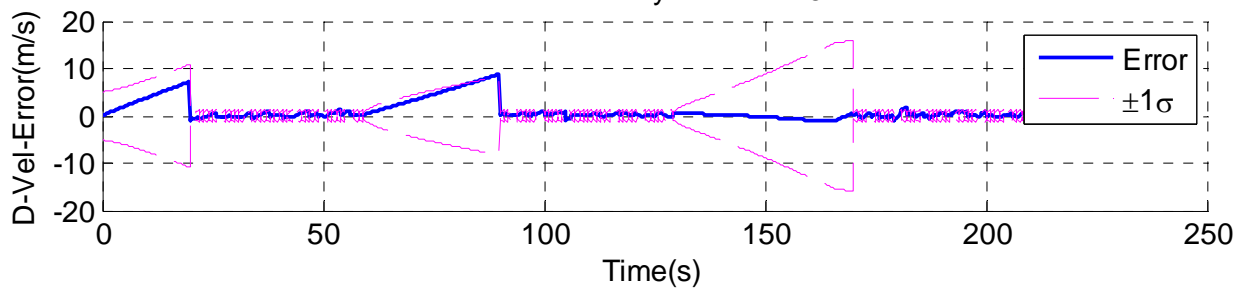

Figure 21 EKF Velocity estimation error and $\pm 1 \sigma$ error bound 


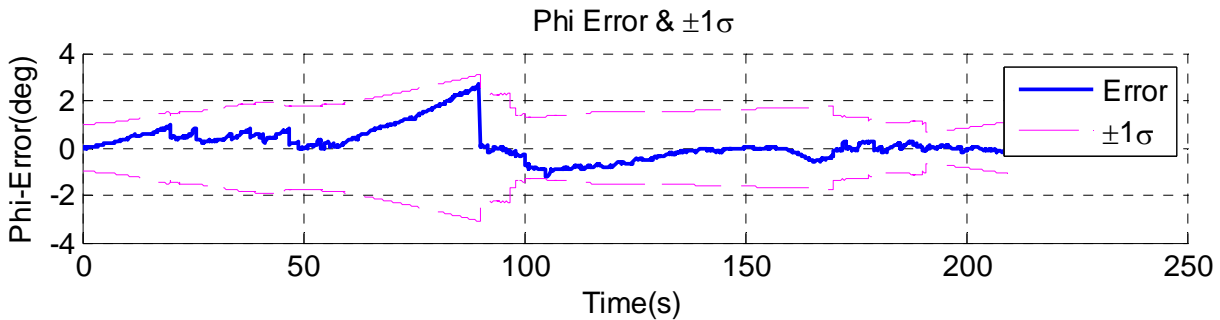

Theta Error \& $\pm 1 \sigma$

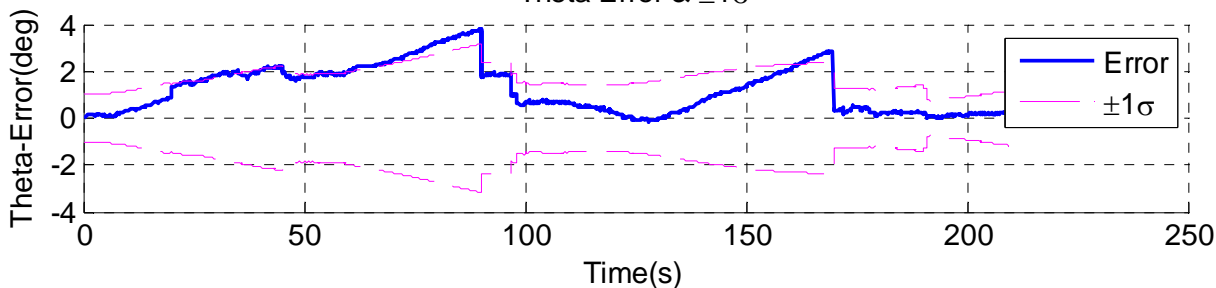

Psi Error \& $\pm 1 \sigma$

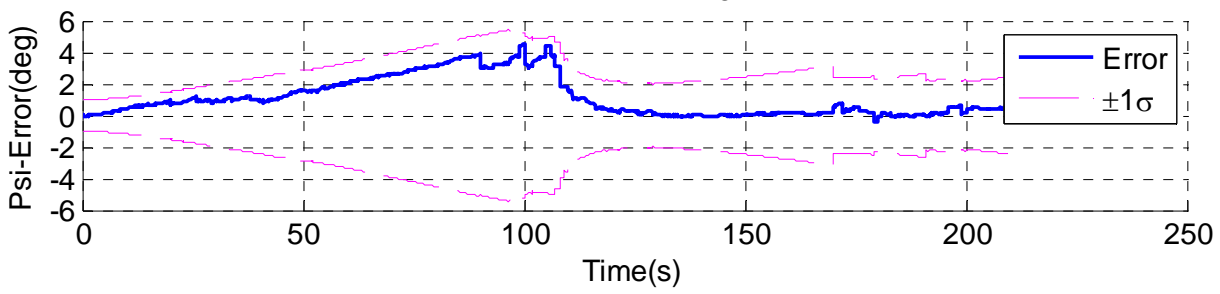

Figure 22 EKF Attitude estimation error and $\pm 1 \sigma$ error bound

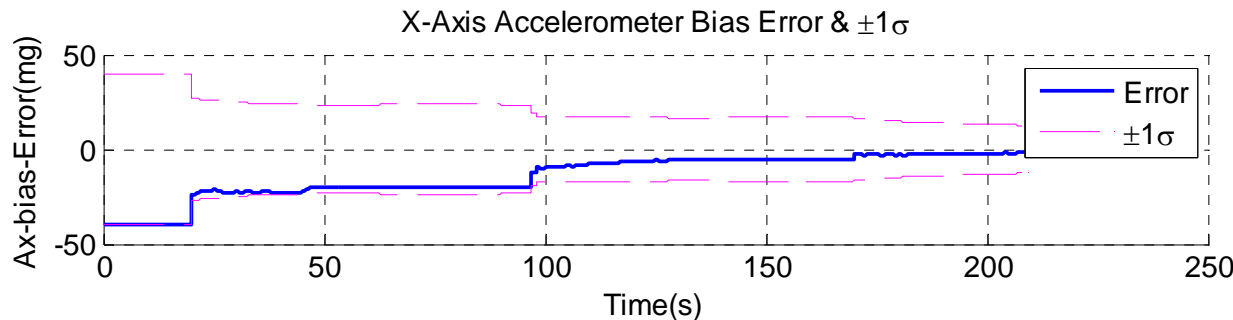

Y-Axis Accelerometer Bias Error $\& \pm 1 \sigma$

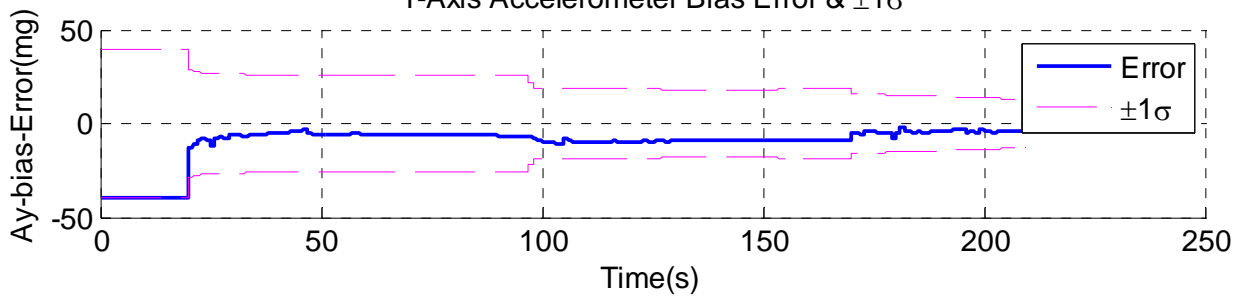

Z-Axis Accelerometer Bias Error $\& \pm 1 \sigma$

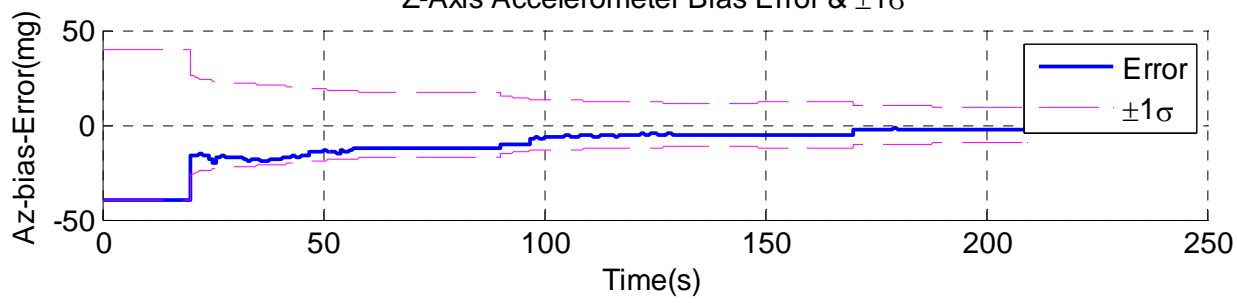

Figure 23 EKF Accelerometer bias estimation error and $\pm 1 \sigma$ error bound 

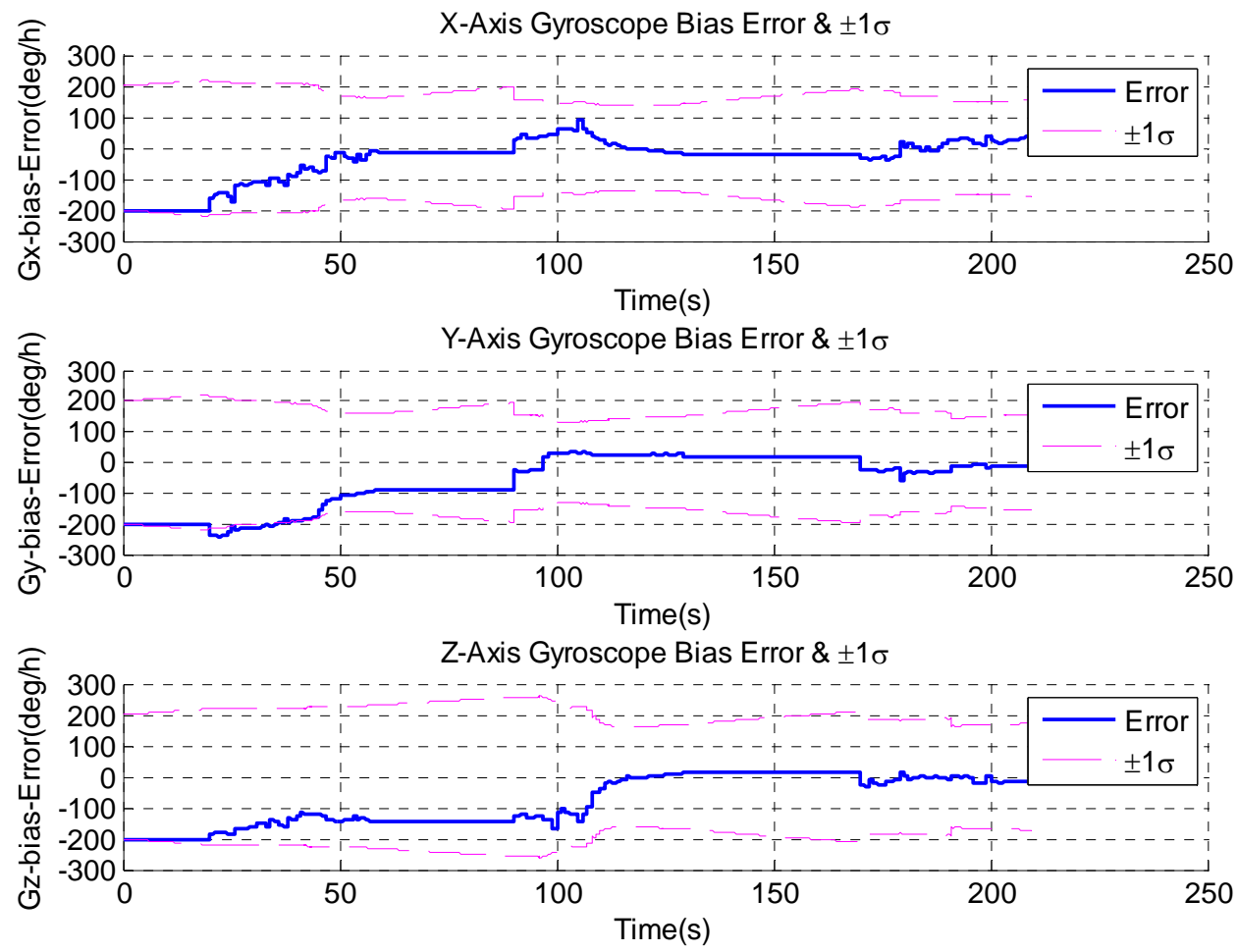

Figure 24 EKF Accelerometer bias estimation error and $\pm 1 \sigma$ error bound
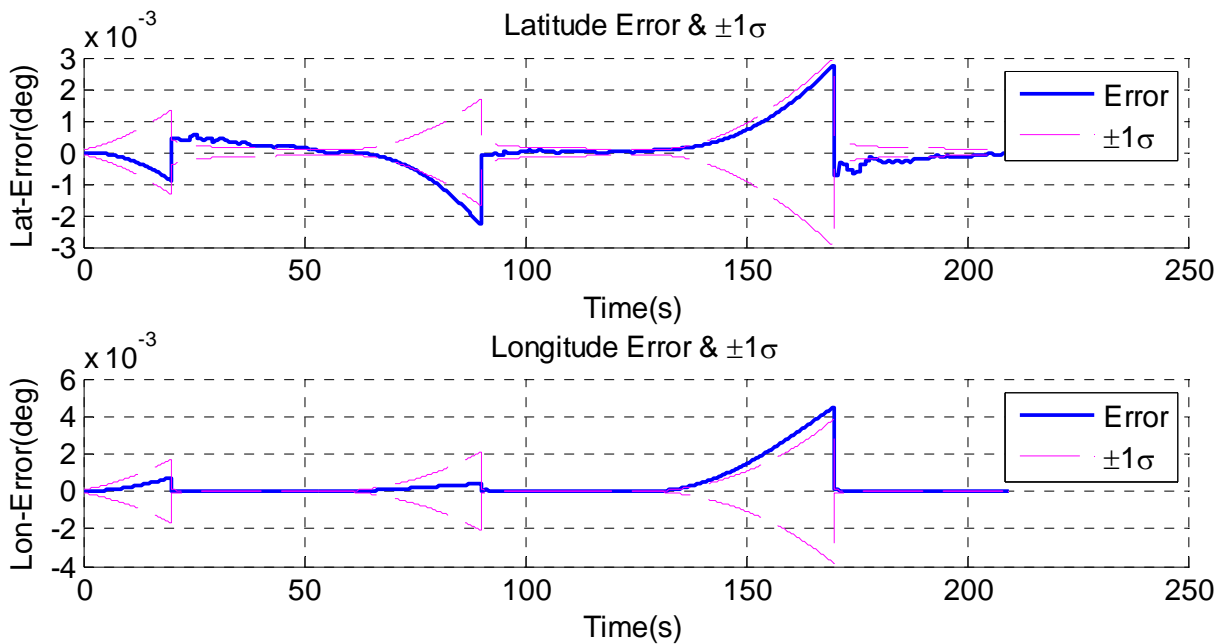

Height Error \& $\pm 1 \sigma$

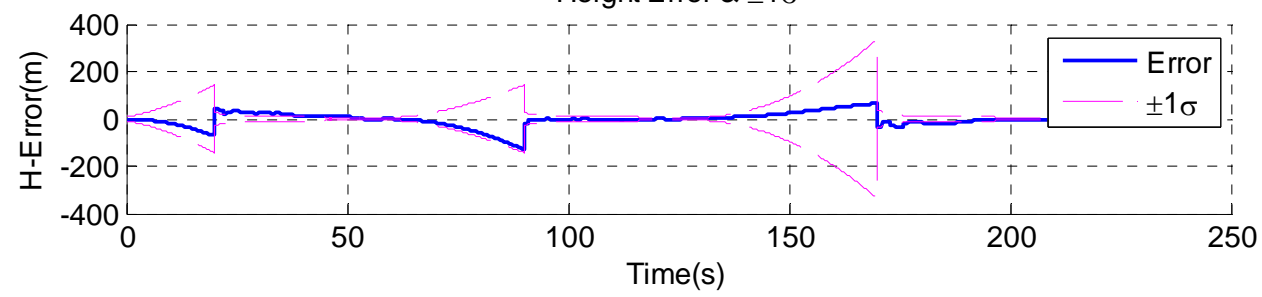

Figure 25 UKF Position estimation error and $\pm 1 \sigma$ error bound 

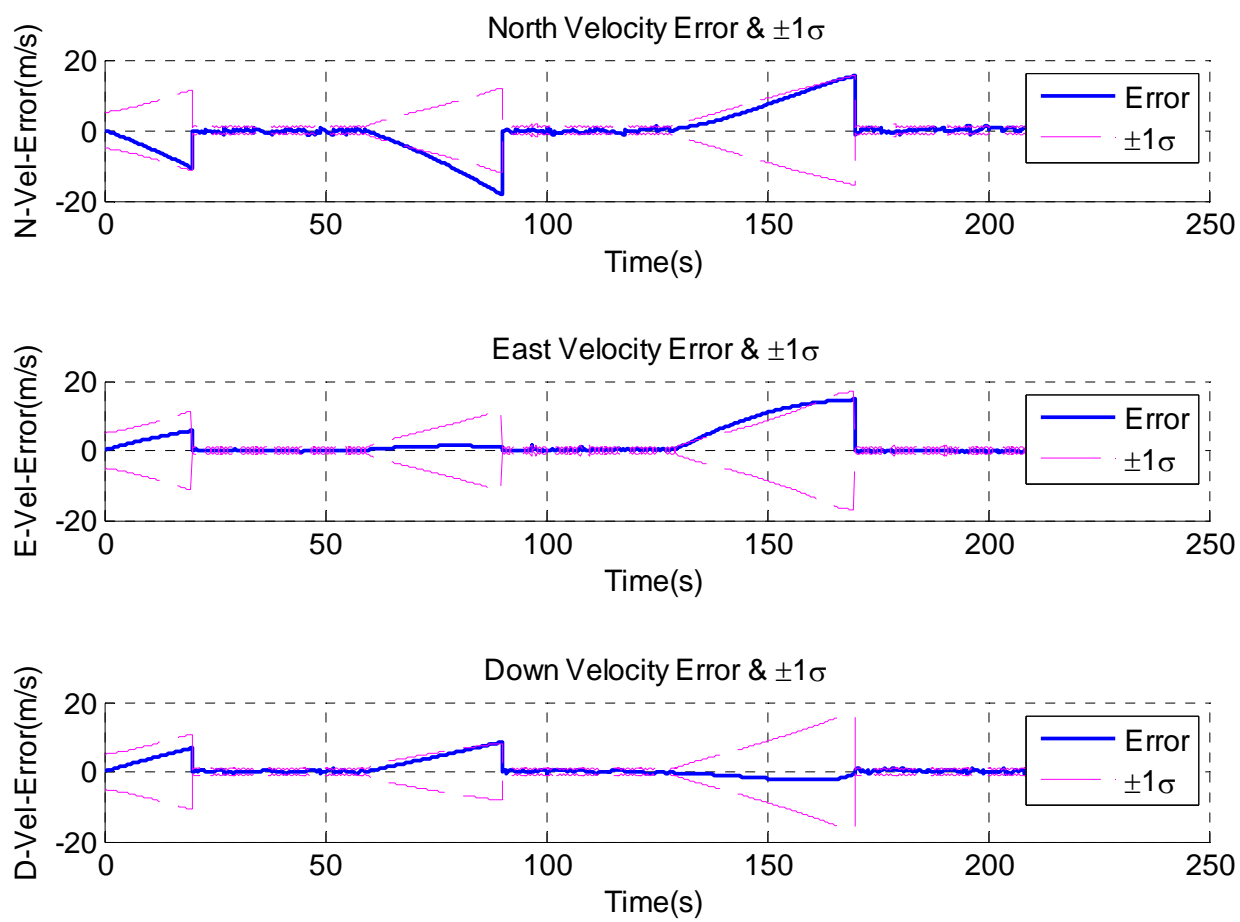

Figure 26 UKF Velocity estimation error and $\pm 1 \sigma$ error bound

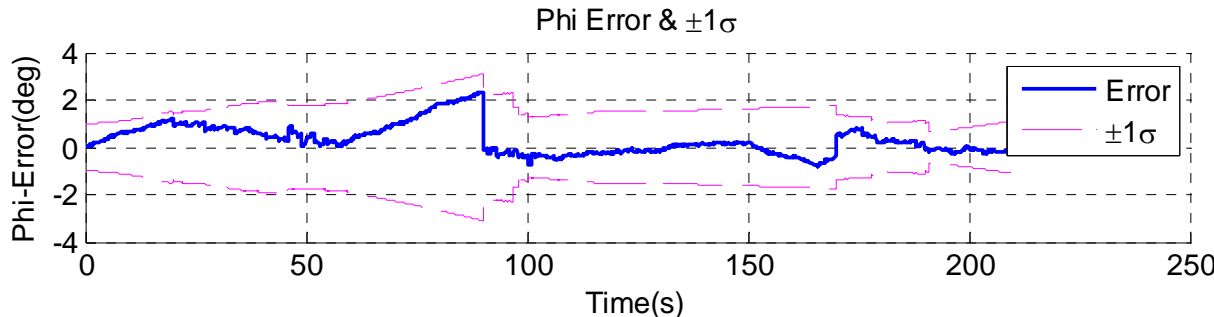

Theta Error \& $\pm 1 \sigma$

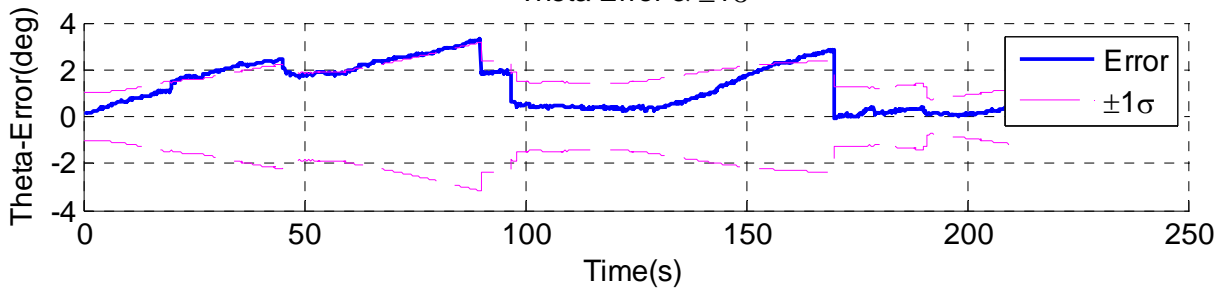

Psi Error $\& \pm 1 \sigma$

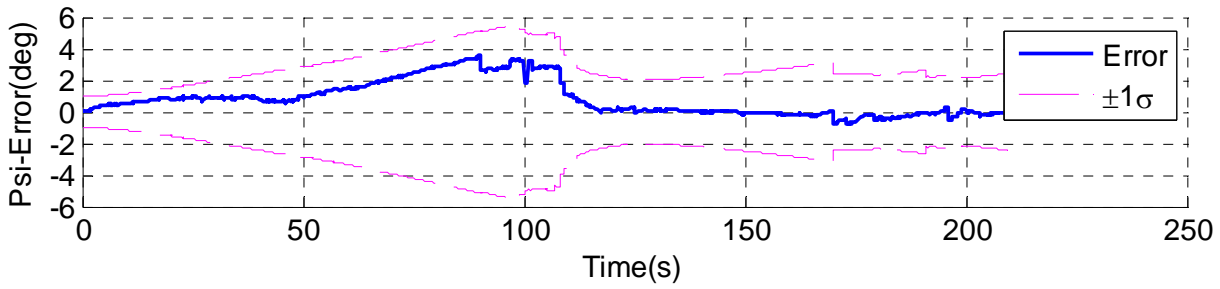

Figure 27 UKF Attitude estimation error and $\pm 1 \sigma$ error bound 


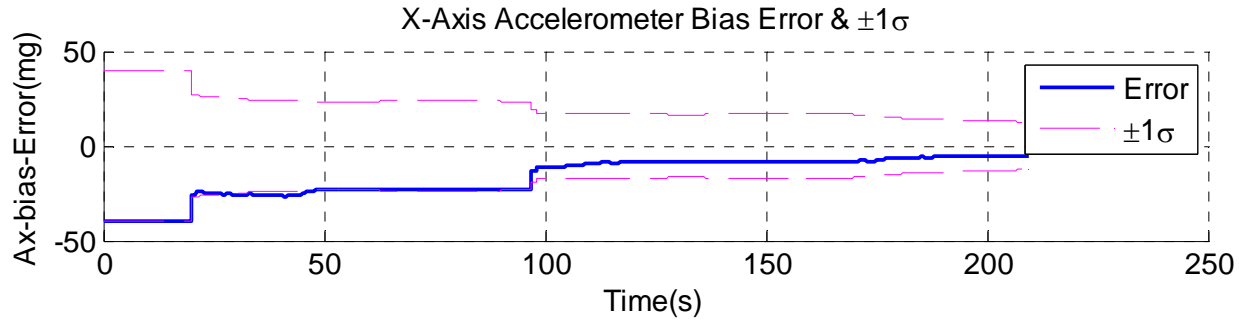

Y-Axis Accelerometer Bias Error $\& \pm 1 \sigma$

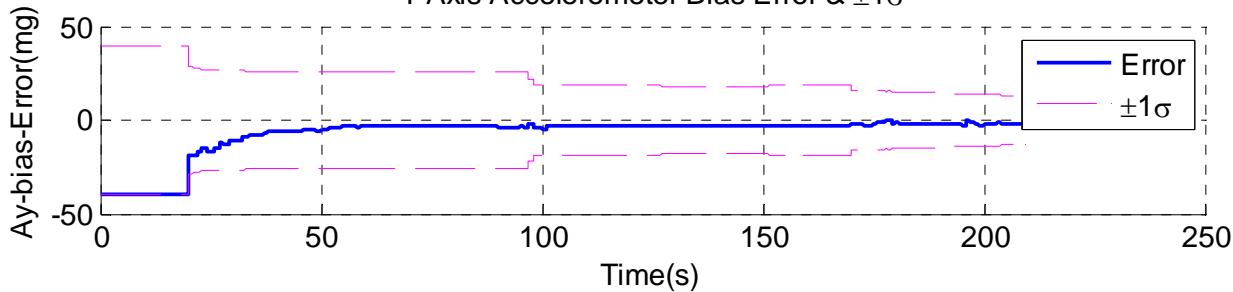

Z-Axis Accelerometer Bias Error $\& \pm 1 \sigma$

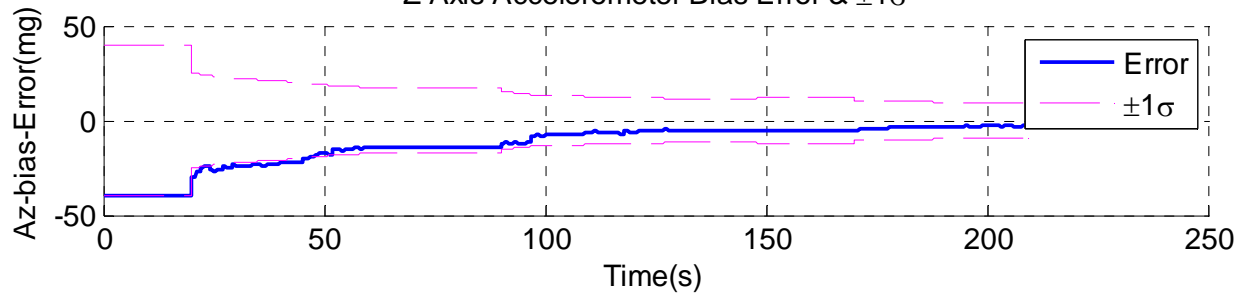

Figure 28 UKF Accelerometer bias estimation error and $\pm 1 \sigma$ error bound

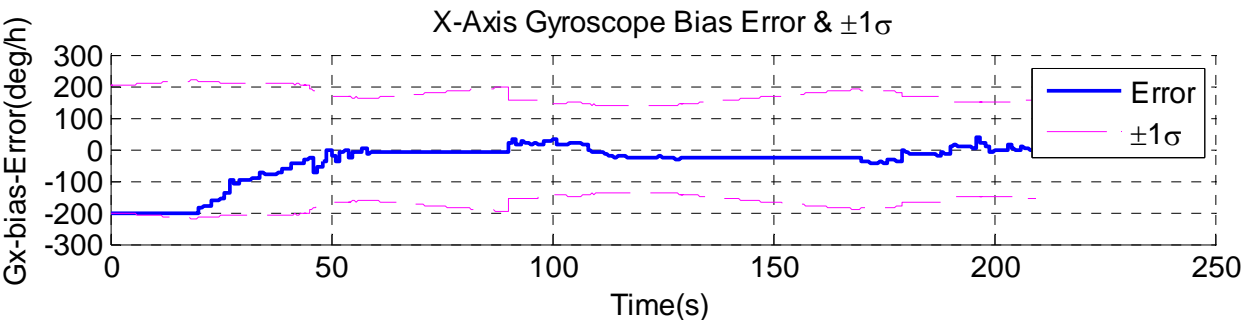

Y-Axis Gyroscope Bias Error $\& \pm 1 \sigma$

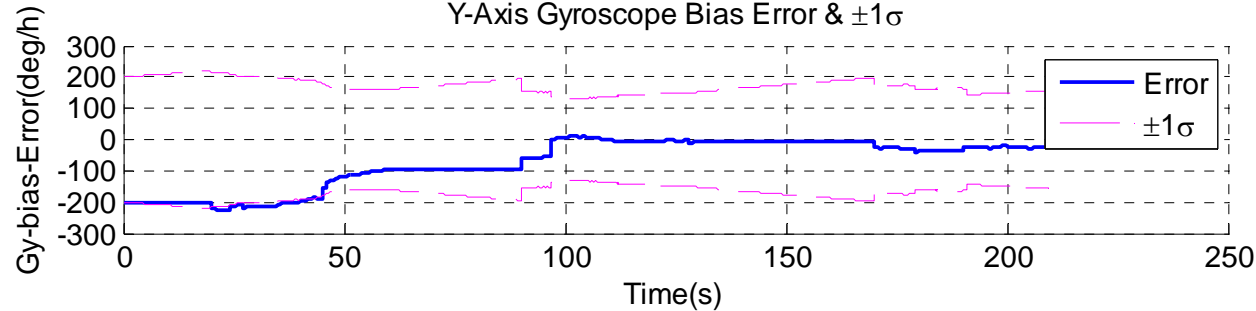

Z-Axis Gyroscope Bias Error $\& \pm 1 \sigma$

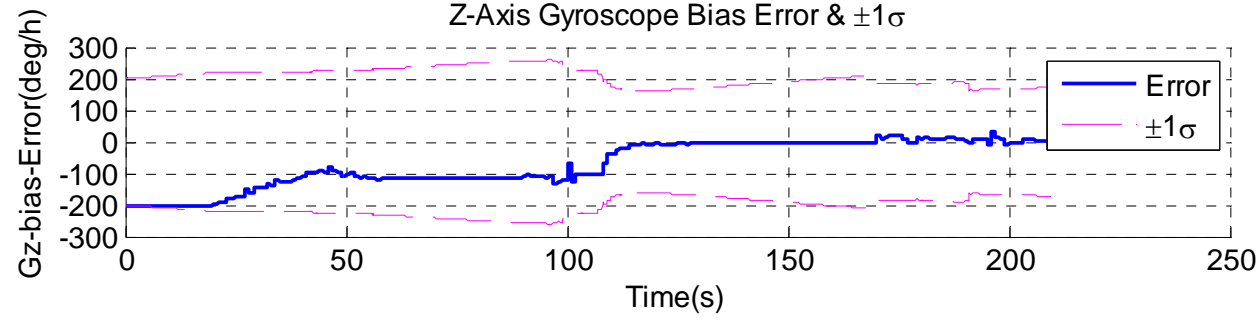

Figure 29 UKF Gyroscope bias estimation error and $\pm 1 \sigma$ error bound 
Performance of the EKF and the UKF algorithms are compared in terms of the Root Mean Square Error (RMSE) metrics which are obtained using the results of Monte Carlo Simulations in which the stochastic IMU and GPS error parameters are set randomly. RMSE of the results are calculated using the Equation (6.1).

$$
R M S E=\frac{1}{N} \sum_{n=1}^{N} \sqrt{\frac{1}{K} \sum_{k=1}^{K}\left(\left(x_{k}-x_{k}^{R E F}\right)^{2}+\left(y_{k}-y_{k}^{R E F}\right)^{2}+\left(z_{k}-z_{k}^{R E F}\right)^{2}\right)}
$$

where $N$ is the number of Monte Carlo runs and $K$ is the number of time steps in the scenario. $\left(x_{k}^{R E F}, y_{k}^{R E F}, z_{k}^{R E F}\right)$ are the error-free reference data and $\left(x_{k}, y_{k}, z_{k}\right)$ are the state estimates of the filter at time step $\mathrm{k}$.

The RMSE results for EKF and UKF are obtained using 1000 Monte Carlo runs. Variation of the two estimators' RMSE performance in time is given in Figure 30.

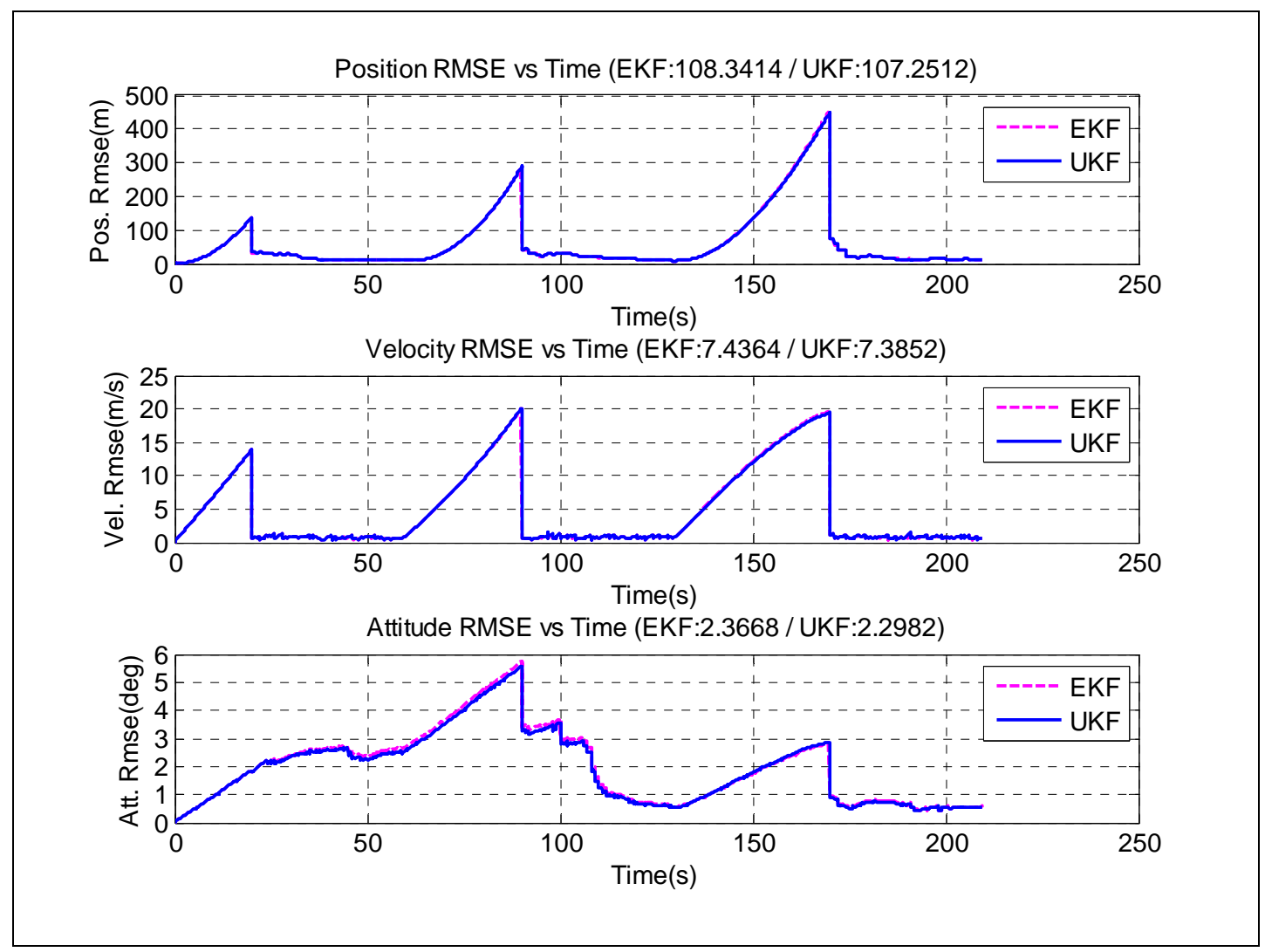

Figure 30 Comparison of EKF and UKF RMSE metrics 
A close look at the Figure 30 shows that the standard UKF slightly improves the performance of the EKF. For comparison, RMSE's of the estimated navigation solutions are given below in Table 13.

Table 13 RMSE Performance comparison of EKF and UKF

\begin{tabular}{|l|c|c|c|}
\hline RMSE & POSITION & VELOCITY & ATTITUDE \\
\hline EKF & 108.3414 & 7.4364 & 2.3668 \\
\hline UKF & 107.2512 & 7.3852 & 2.2982 \\
\hline Improvement & $1.01 \%$ & $0.689 \%$ & $2.89 \%$ \\
\hline
\end{tabular}

However, when different segments of the flight trajectory are considered separately, UKF does not have superior performance at all times. This case can be observed by simply zooming in to Figure 30 . While the position RMSE of the UKF is barely larger than that of the EKF at the end of 20 seconds GPS outage, UKF has better performance than the EKF at the end of 30 and 40 seconds of GPS outage periods as seen in Figure 31.

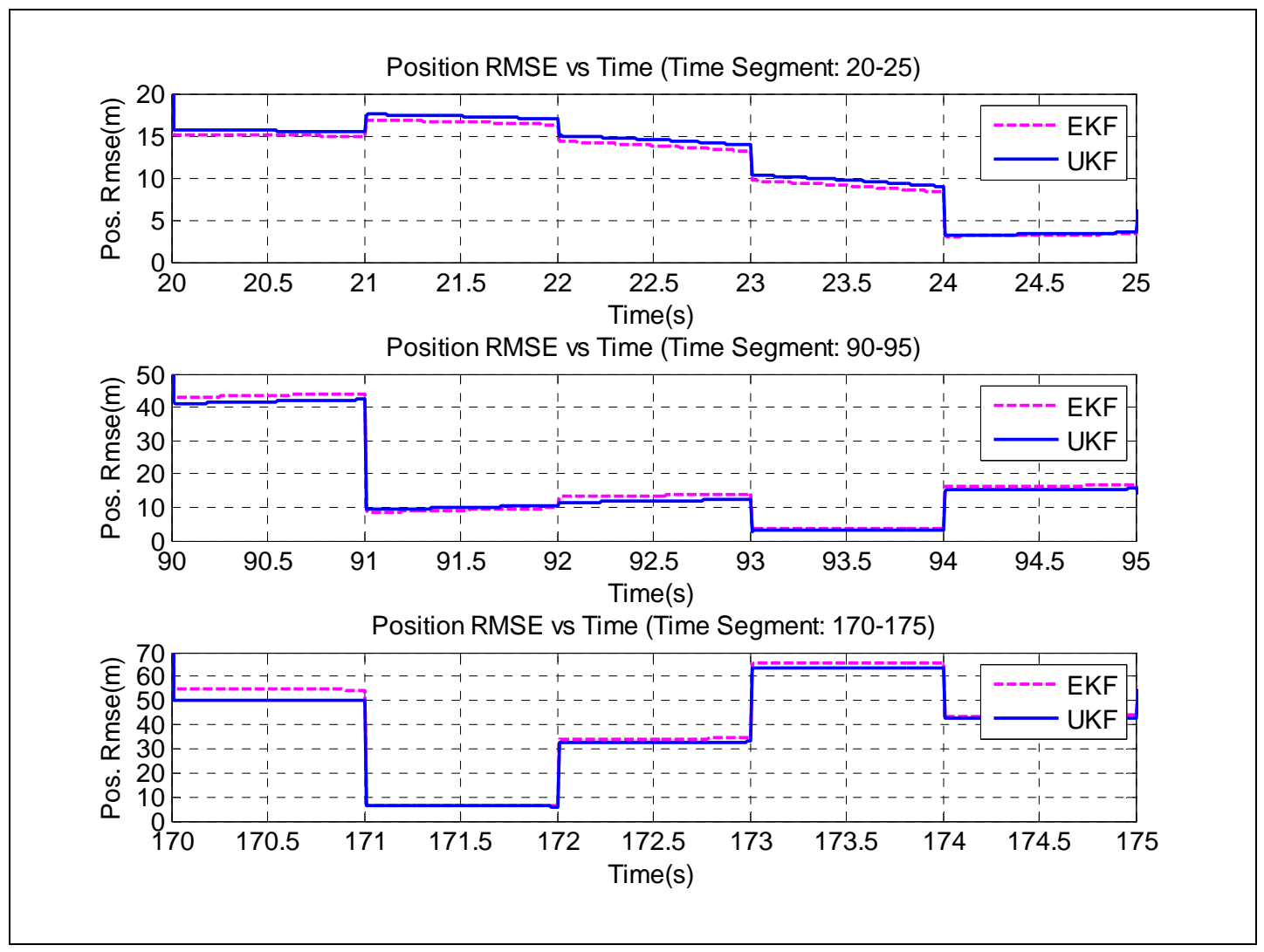

Figure 31 Position RMSE values of EKF\&UKF (Zoomed version of Figure 30) 
Therefore, selection of a single SUT parameter in the UKF throughout the trajectory does not results in superior performance at all time.

\subsubsection{Adaptive UKF Results}

The adaptive scheme discussed in Section 5.4 is used in the same flight trajectory and results of the proposed method are compared with the standard EKF and UKF implementations. An additional Monte Carlo Analysis is made for obtaining the results of the AUKF algorithm. The simulation results can be seen in Table 14 in terms of RMSE performance of the estimator. When different flight segments are investigated separately, it is observed that the correct choice of the SUT parameter at specific covariance bounds brings better performance compared to using the same parameters throughout the scenario. While evaluating the performances of three methods, EKF is selected as the basis for comparison. Performance improvements of the AUKF with respect to the EKF and UKF are calculated in percentages and the following results are obtained.

Table 14 RMSE Performance comparison of EKF, UKF and AUKF

\begin{tabular}{|l|c|c|c|}
\hline RMSE & POSITION & VELOCITY & ATTITUDE \\
\hline EKF & 108.3414 & 7.4364 & 2.3668 \\
\hline UKF & 107.2512 & 7.3852 & 2.2982 \\
\hline AUKF & 105.1268 & 7.3647 & 2.2764 \\
\hline Improvement Over UKF & $1.98 \%$ & $0.28 \%$ & $0.95 \%$ \\
\hline Improvement Over EKF & $2.97 \%$ & $0.96 \%$ & $3.82 \%$ \\
\hline
\end{tabular}

Table 14 reveals that, standard UKF slightly improves the navigation performance. The proposed method further introduces a little increase in the performance of the navigation system by changing the UT parameters adaptively depending on the recent covariance of the estimator. 


\subsection{Field Test Results}

A field test is conducted using a tactical grade INS/GPS integrated system as the reference and a stabilization grade low performance IMU as the unit under test. These two systems can be seen in Figure 32.

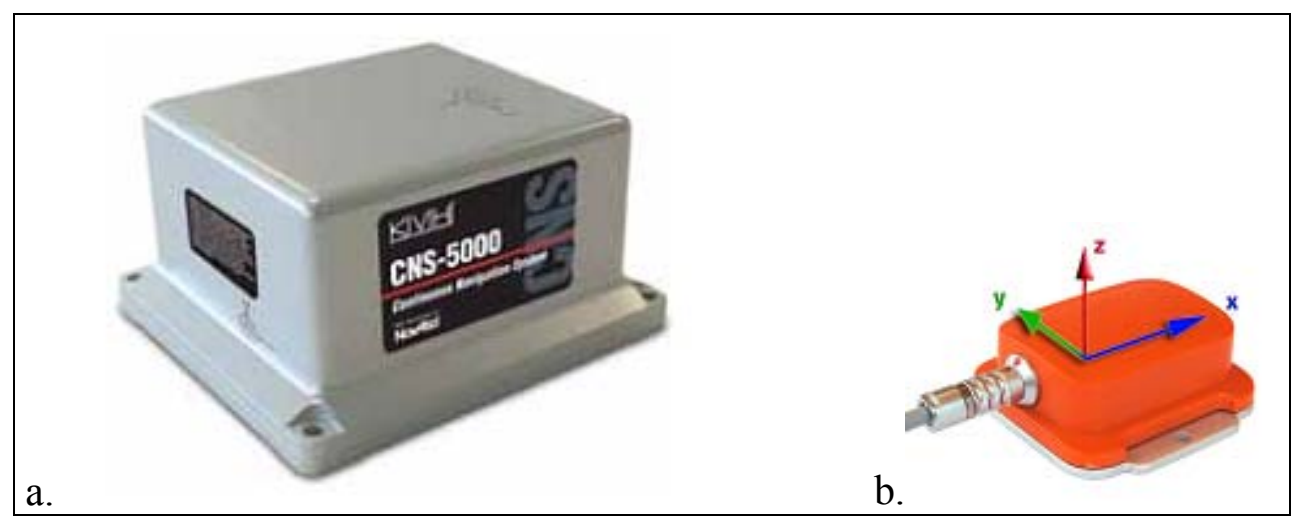

Figure 32 Navigation Systems: a. FOG INS/GPS- CNS5000, b. MEMS IMU-MTi

CNS5000 is a high performance INS/GPS integrated navigation system of KVH Industries Inc. which uses a tactical grade FOG IMU. MTi is a low cost - low performance MEMS IMU of Xsens Technologies Inc.

In the test setup, both of the navigation systems are located in a passenger car side by side and GPS antenna of the reference system is attached on the top of the car. An overview of the test set-up for the reference system is given in the following figure. The utilized MEMS IMU is directly connected to the USB port of the test-PC. 


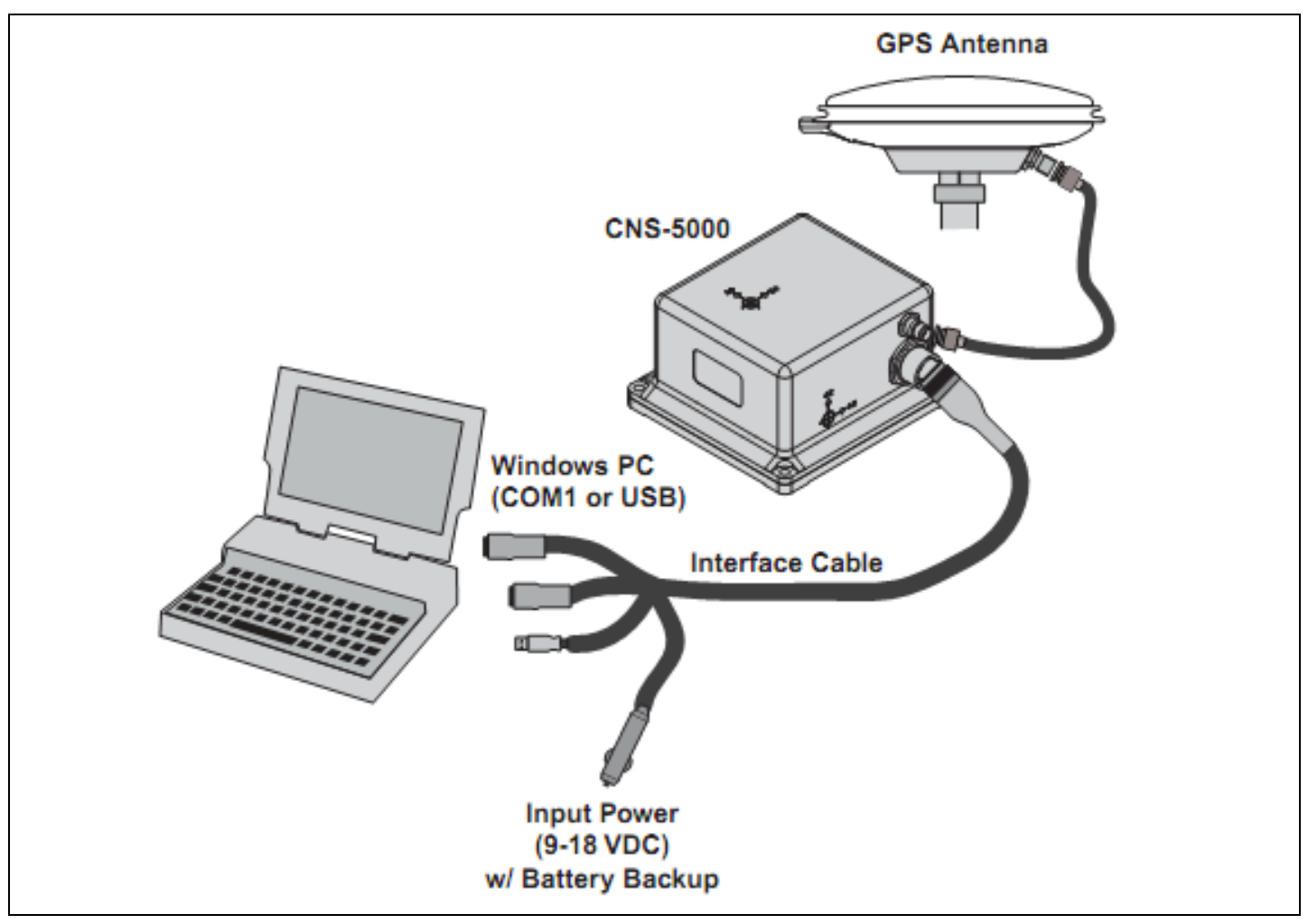

Figure 33 Test set-up for the reference system (Figure is adapted from [38])

In the rest of the thesis, measurements and navigation results of the high performance INS/GPS integrated system CNS5000 is referred to as reference (REF in short). Outputs of the low performance IMU MTi is referred to as MEMS.

In Figure 34 and Figure 35, linear acceleration and angular rate measurements of the two navigation units are given, respectively. 


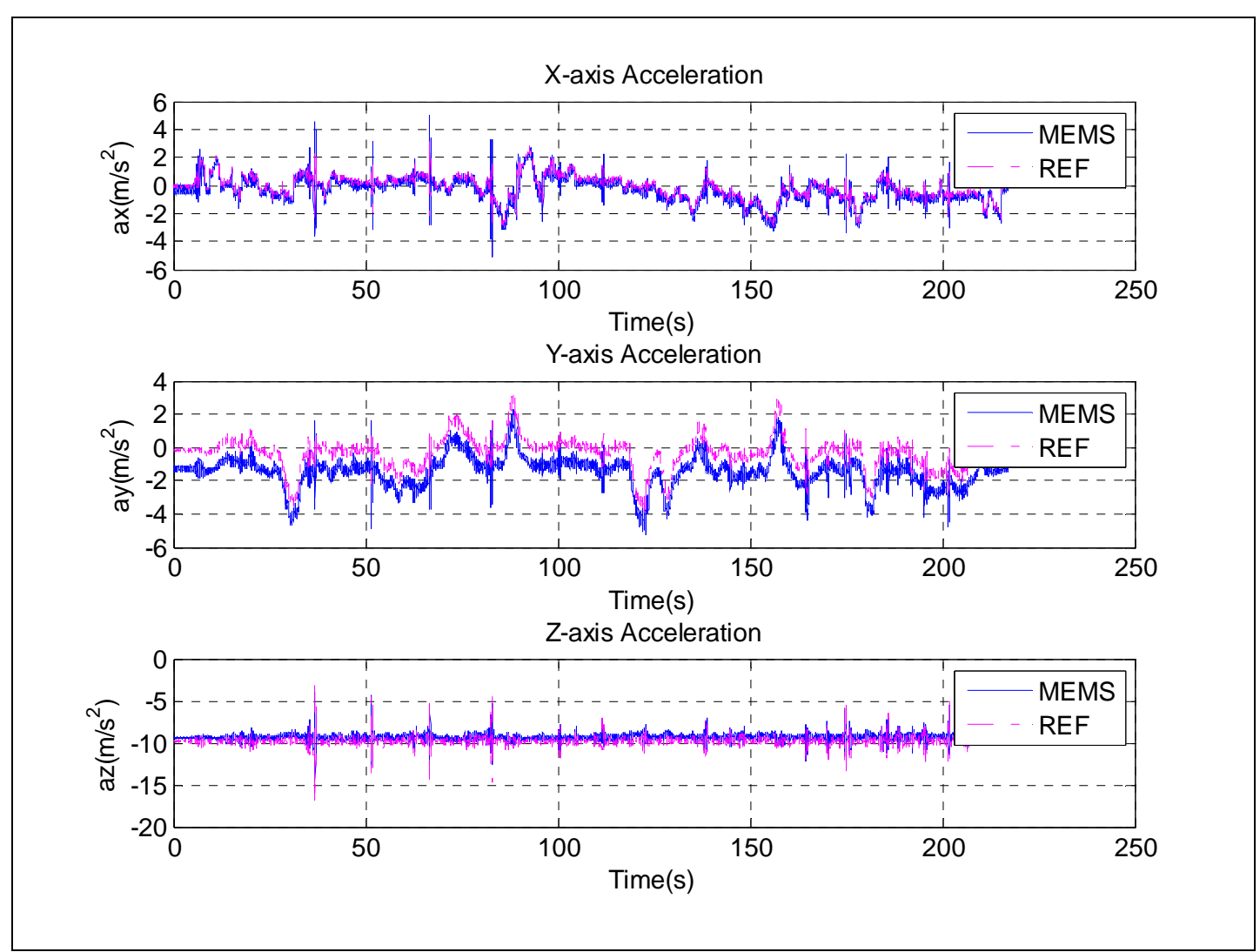

Figure 34 Acceleration measurements of CNS5000 (REF) and MTi (MEMS)

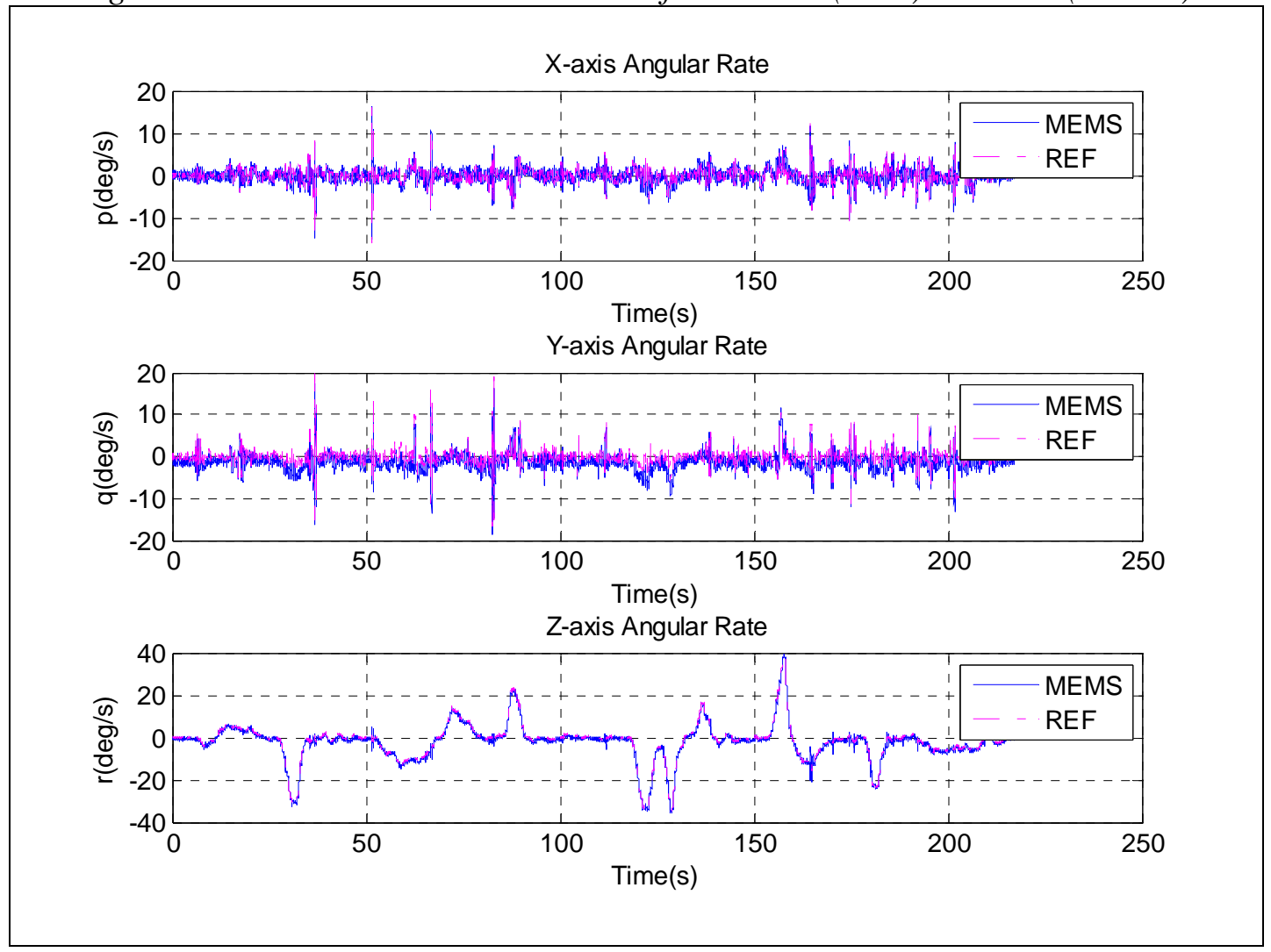

Figure 35 Angular rate measurements of CNS5000 (REF) and MTi (MEMS) 


\subsubsection{Test Results without GPS Outages}

Navigation results namely position, velocity and attitude of the test vehicle obtained by AUKF algorithm are given together with the reference system outputs in Figure 36 toFigure 40 .

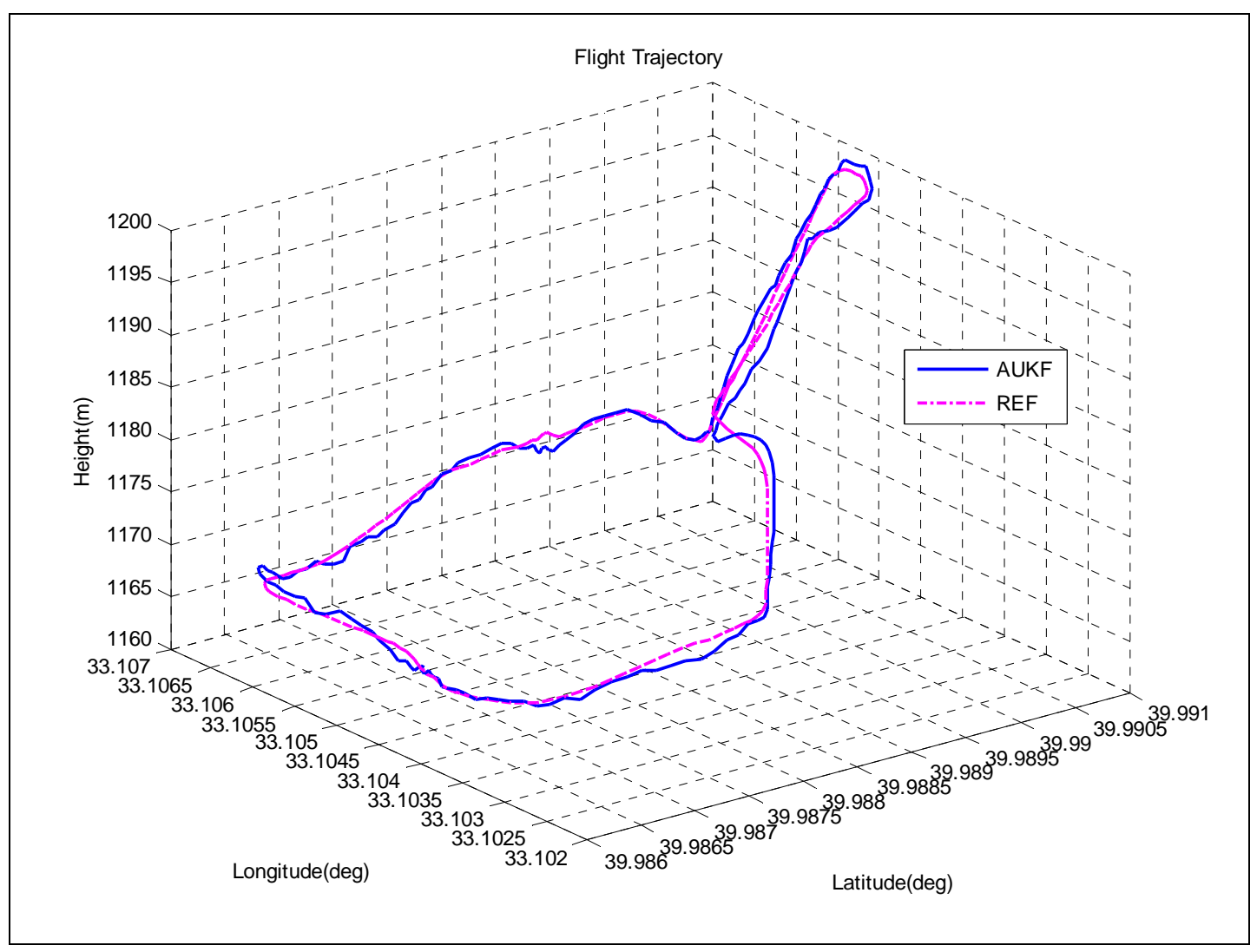

Figure 36 3D Flight trajectory computed by the reference system and AUKF 


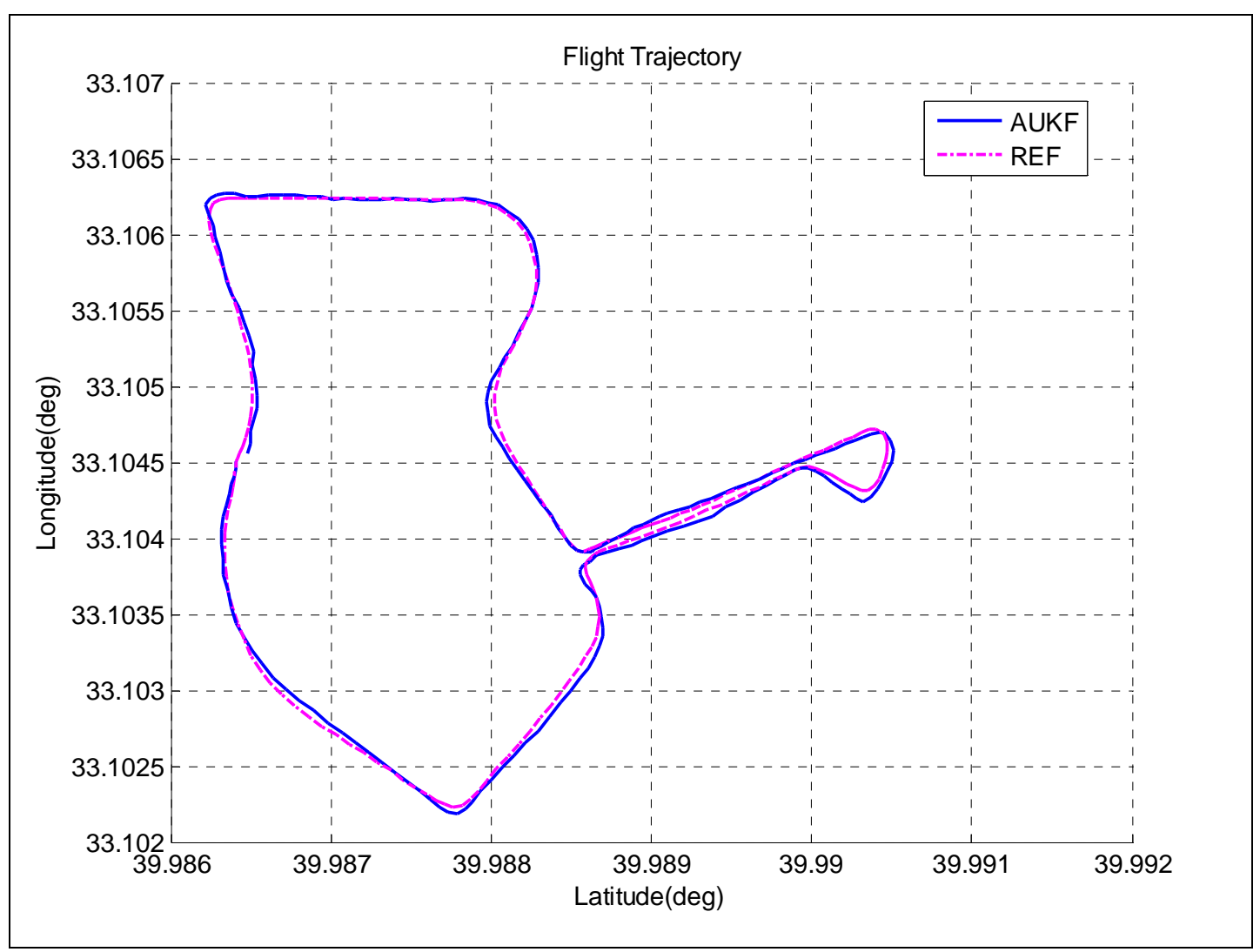

Figure 37 Horizontal position computed by the reference system and AUKF

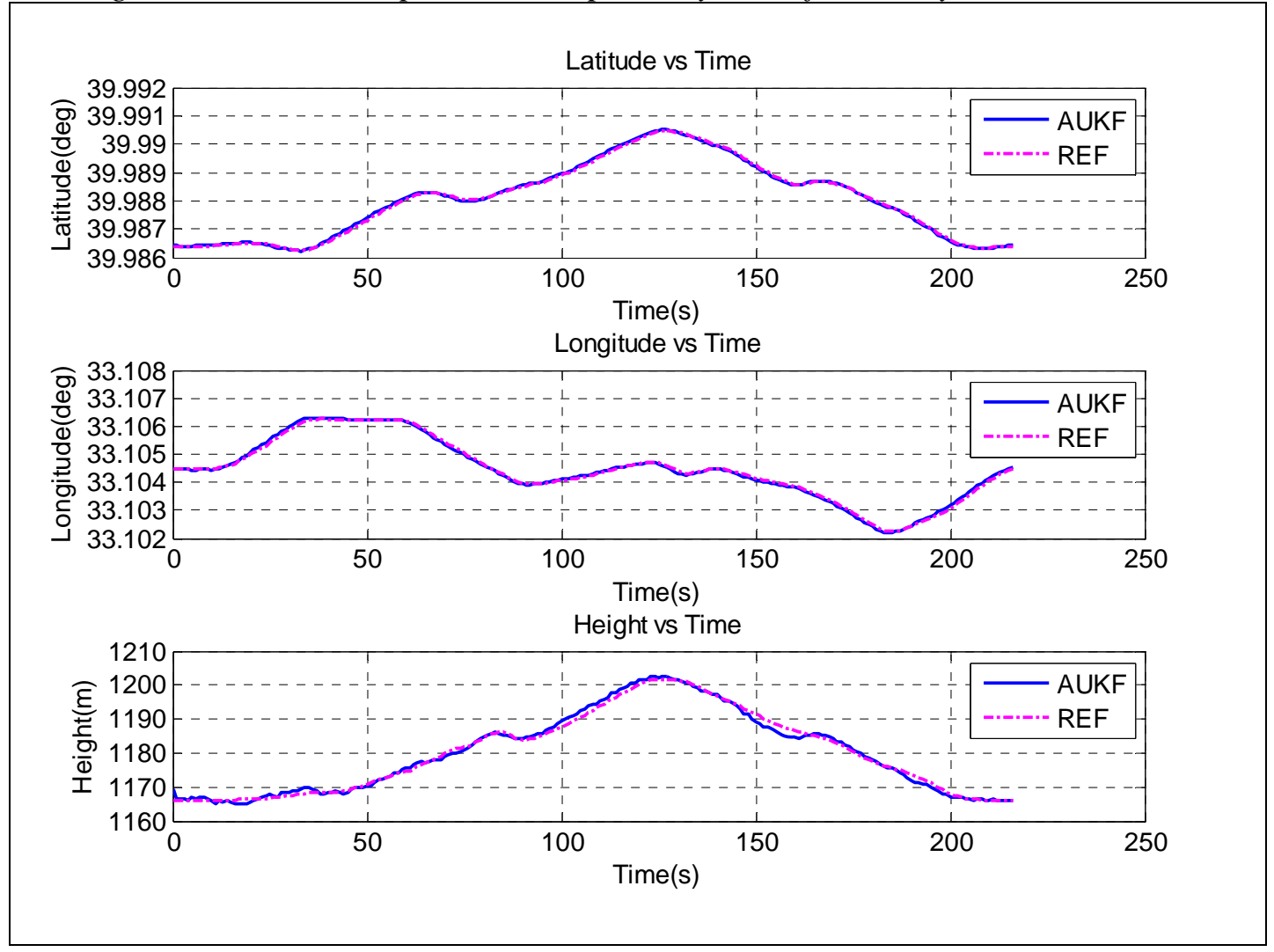

Figure 38 Position output computed by the reference system and AUKF 


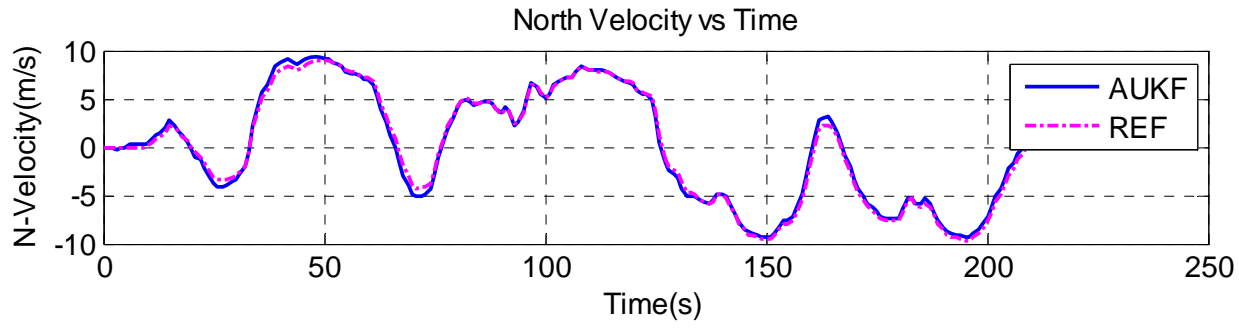

East Velocity vs Time

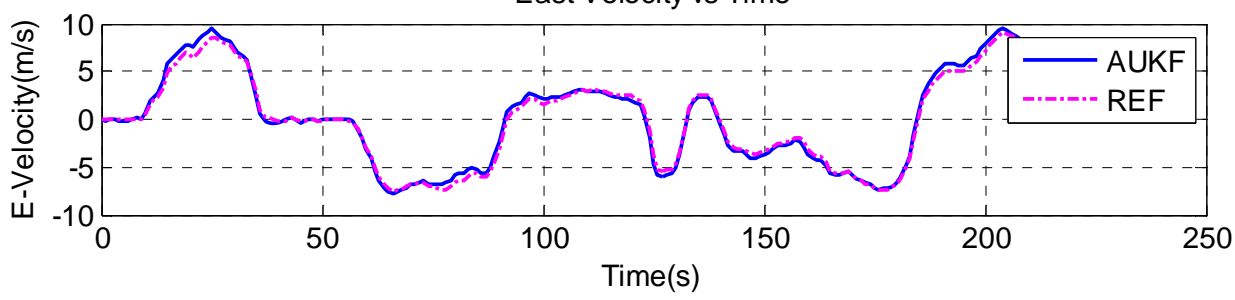

Down Velocity vs Time

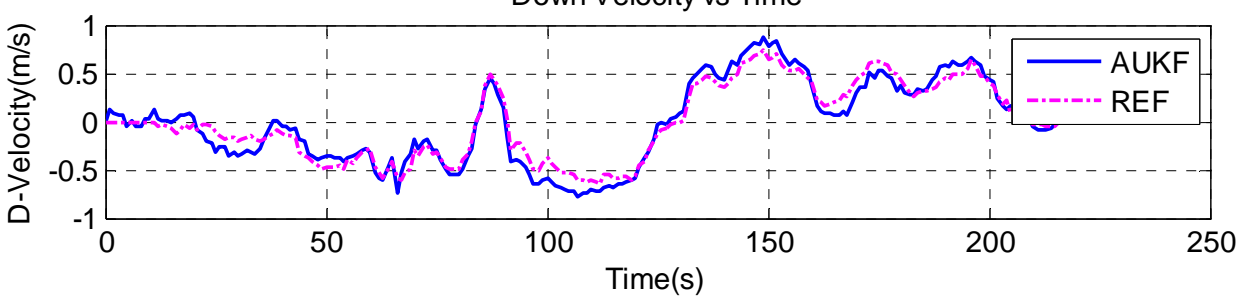

Figure 39 Velocity output computed by the reference system and AUKF
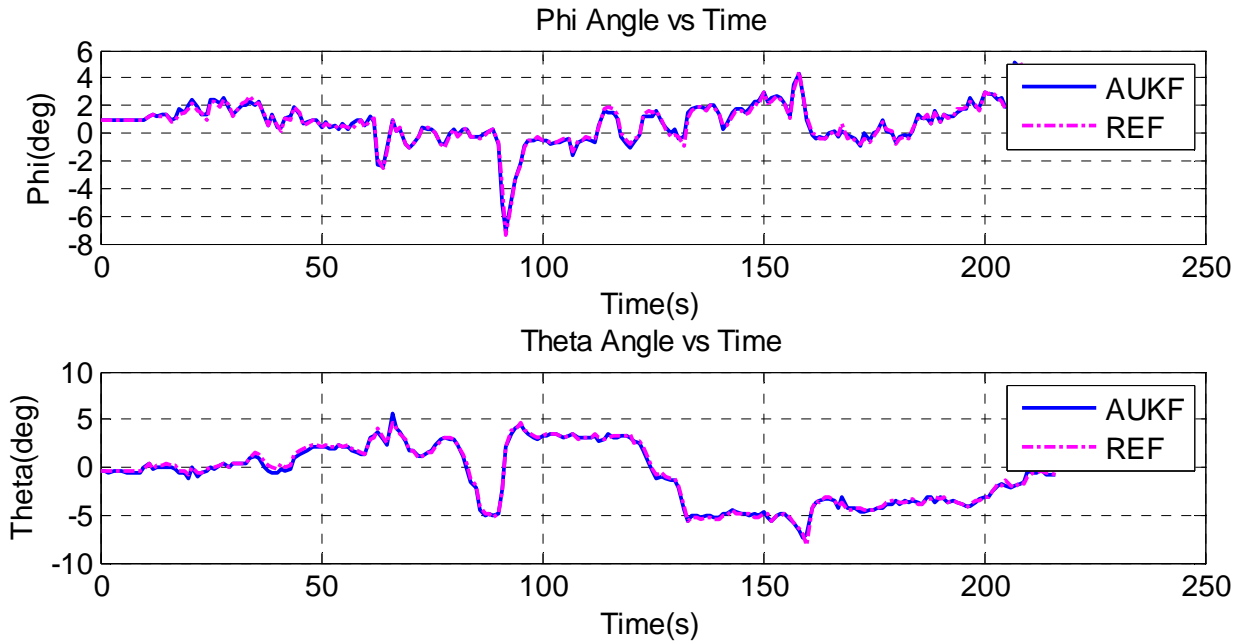

Psi Angle vs Time

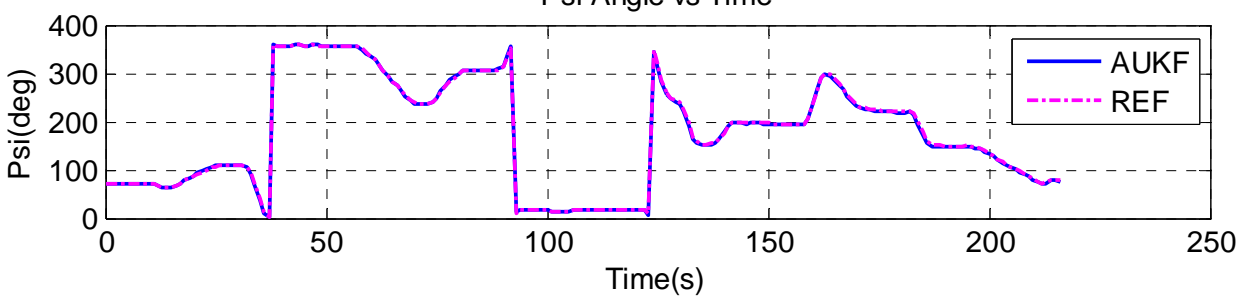

Figure 40 Attitude output computed by the reference system and AUKF 
Performance comparison of EKF, UKF and AUKF algorithms for the field test with continuous GPS aiding is given in Table 15.

Table 15 Field test RMSE performance comparison of EKF, UKF and AUKF

\begin{tabular}{|l|c|c|c|}
\hline RMSE & POSITION & VELOCITY & ATTITUDE \\
\hline EKF & 8.78 & 0.74 & 1.1 \\
\hline UKF & 8.65 & 0.72 & 0.94 \\
\hline AUKF & 8.63 & 0.73 & 0.83 \\
\hline Improvement Over UKF & $0.23 \%$ & $-1.4 \%$ & $1.1 \%$ \\
\hline Improvement Over EKF & $1.7 \%$ & $1.3 \%$ & $1.5 \%$ \\
\hline
\end{tabular}

When GPS aiding is uninterrupted, it can be deduced that UKF does not have much improvement over the standard INS/GPS integration algorithm EKF [39]. By further tuning the EKF carefully, performances of the three filters would become closer to each other.

\subsubsection{Test Results with GPS Outages}

Similar to the simulations discussed in Section 6.1, three GPS outage periods are created artificially in the post processing of the data collected at field tests. The time periods given in Table 16 are used as the GPS outage periods.

Table 16 GPS outage periods in the flight trajectory

\begin{tabular}{|c|c|c|c|c|c|c|}
\hline GPS Availability & OFF & ON & OFF & ON & OFF & ON \\
\hline Time Period (s) & $0-20$ & $20-60$ & $60-90$ & $90-130$ & $130-170$ & $170-210$ \\
\hline
\end{tabular}

At the GPS outage segments of the test, measurement update routines of the estimators are skipped. Navigation errors and 1 sigma standard deviation bounds of the formed scenario are given in Figure 41 to Figure 44. Navigation errors given in these figures are calculated by comparing the results of the AUKF integration routine with the reference INS/GPS integrated system. In this computation, inertial outputs of the MEMS IMU are utilized. 


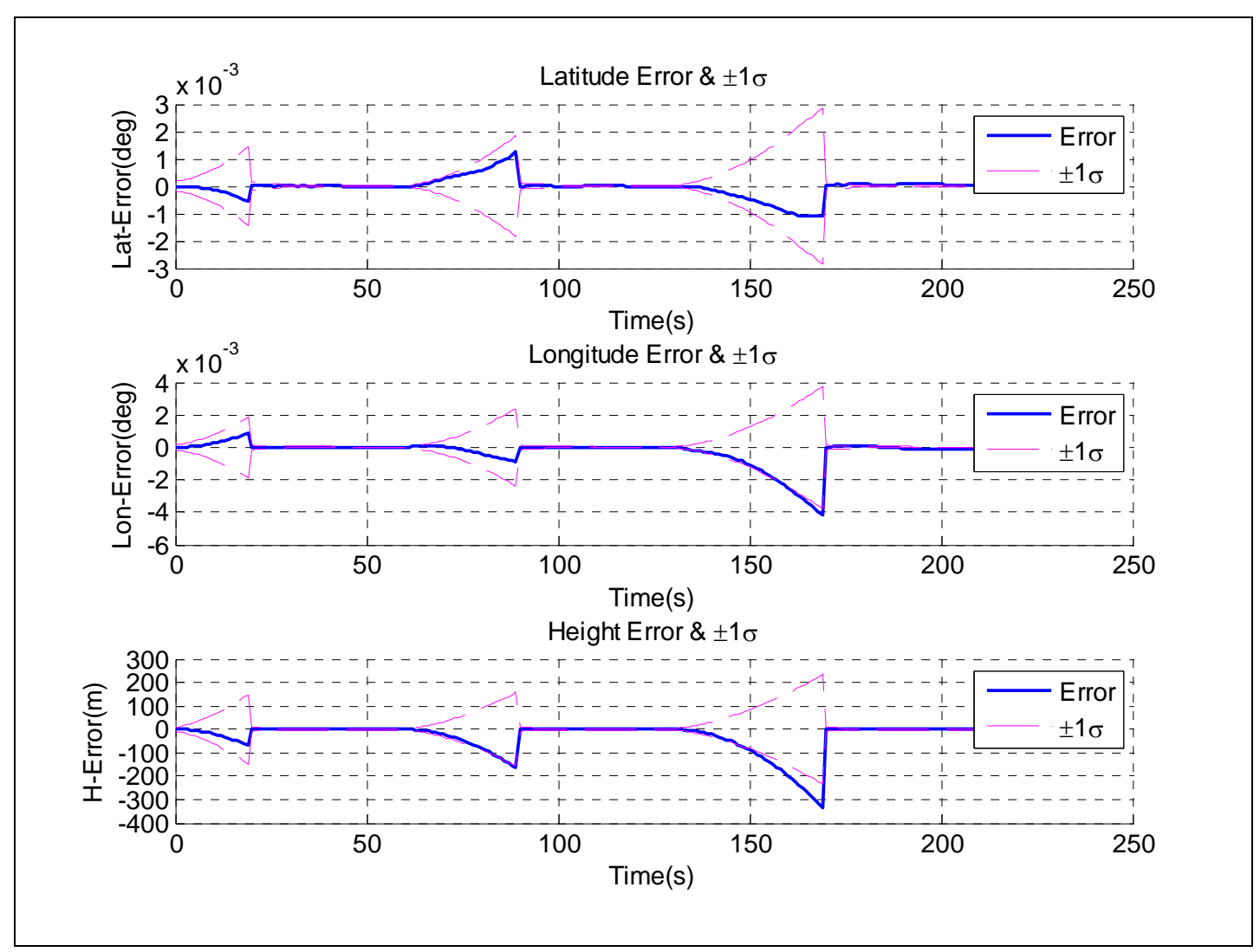

Figure 41 AUKF Position estimation error and $\pm 1 \sigma$ error bound

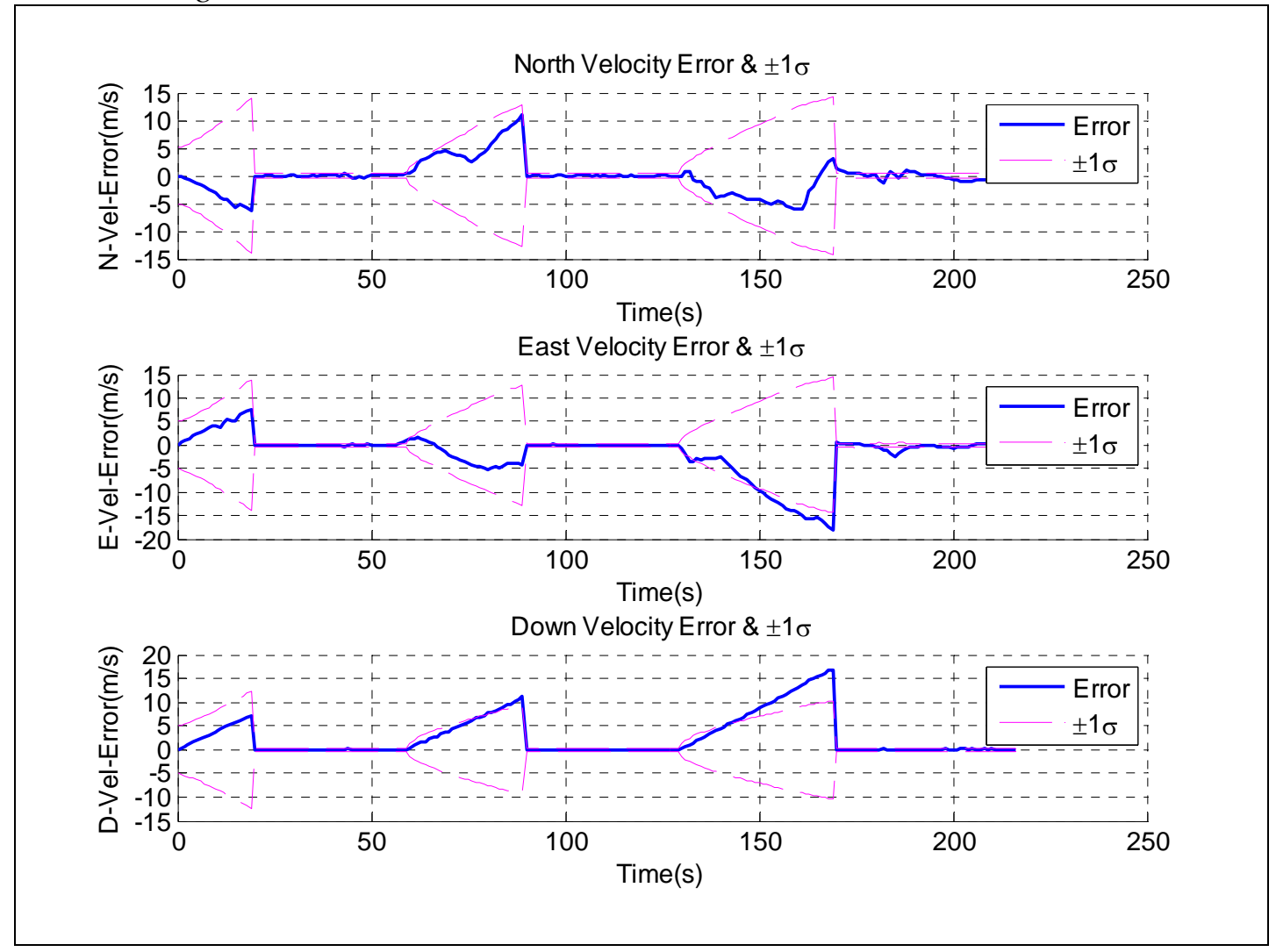

Figure 42 AUKF Position estimation error and $\pm 1 \sigma$ error bound 


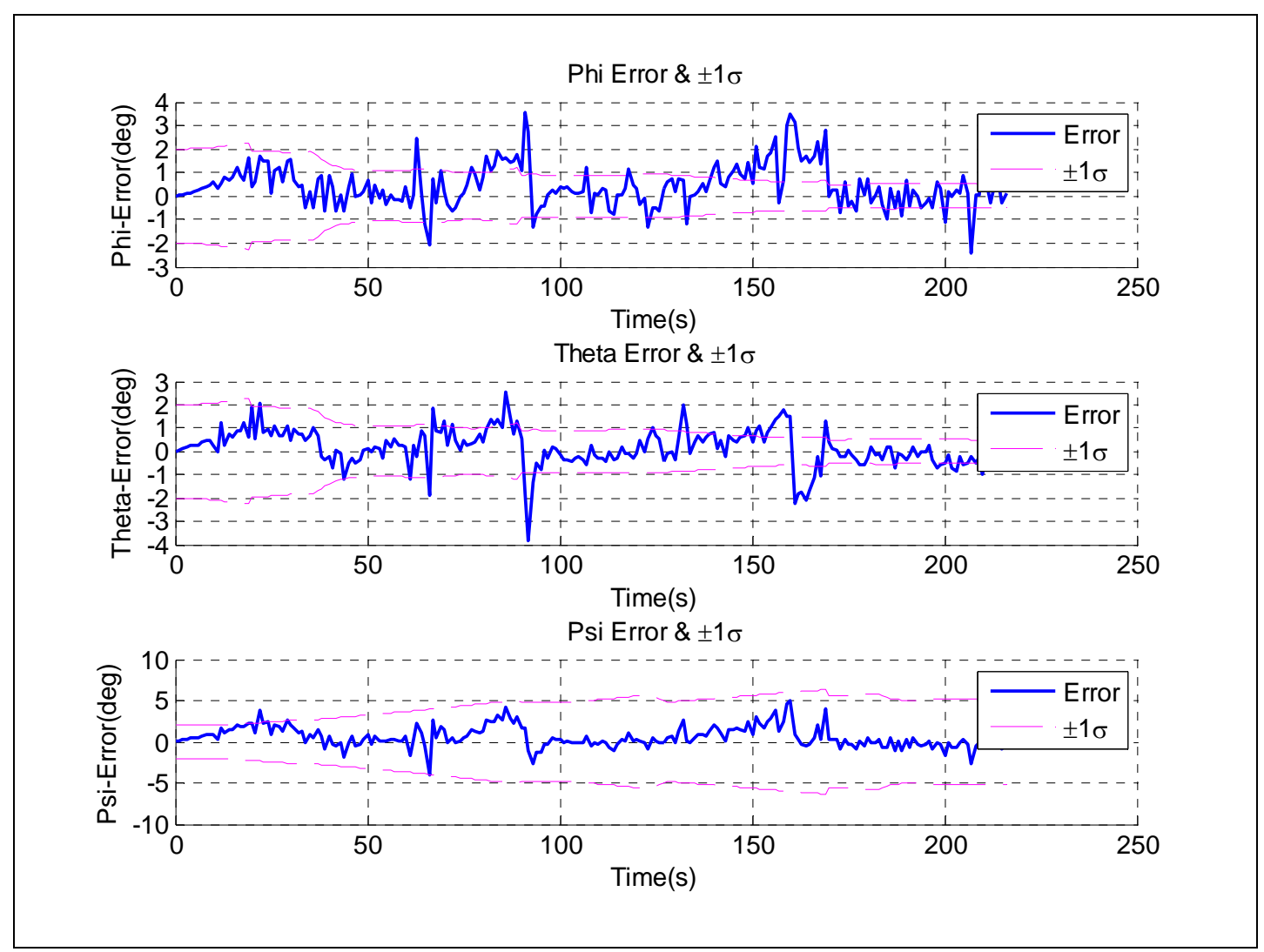

Figure 43 AUKF Position estimation error and $\pm 1 \sigma$ error bound
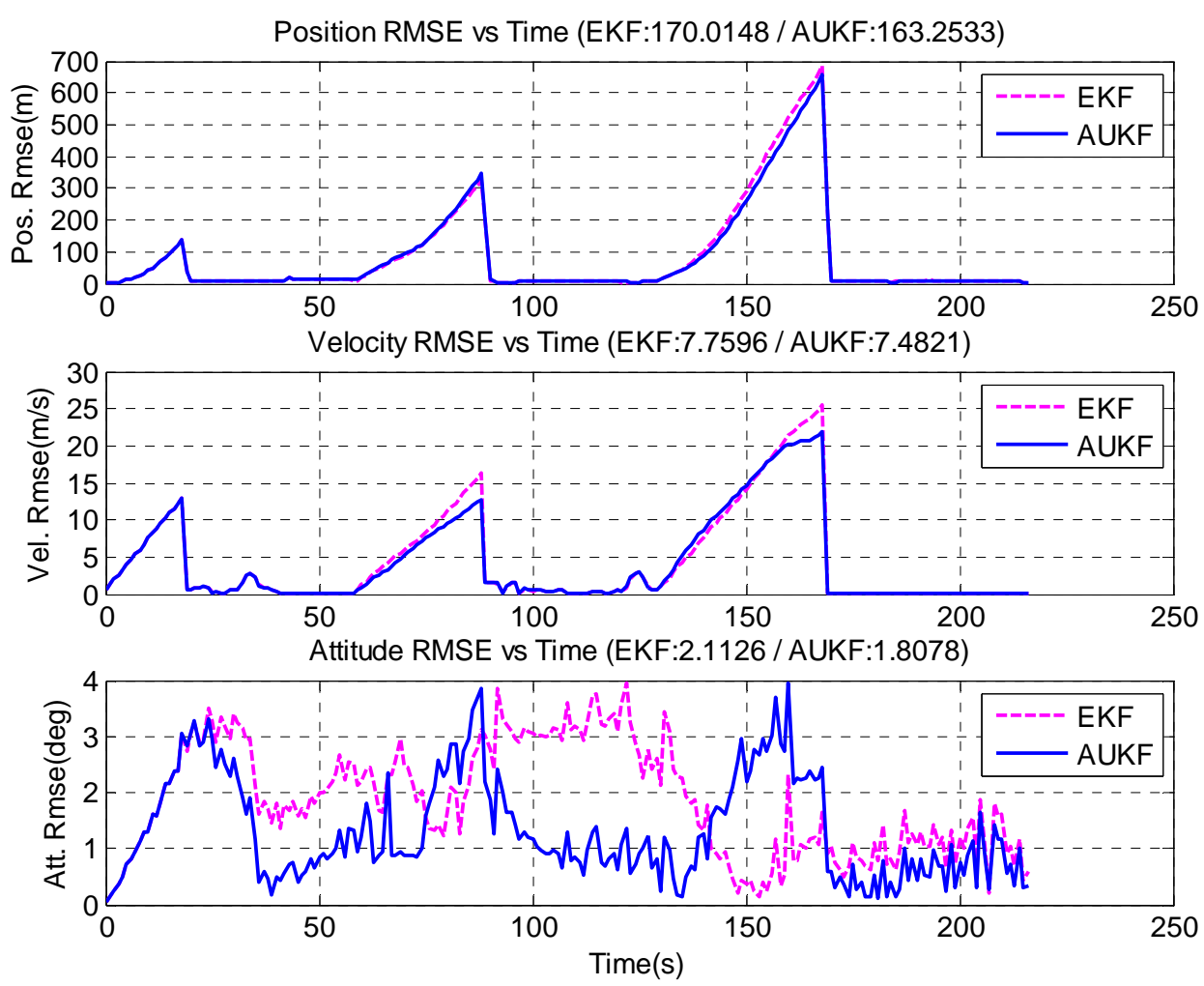

Figure 44 AUKF Position estimation error and $\pm 1 \sigma$ error bound 
A summary of the results obtained from the collected data is given in Table 17 .

Table 17 Field test RMSE performance comparison of EKF, UKF and AUKF

\begin{tabular}{|l|c|c|c|}
\hline RMSE & POSITION & VELOCITY & ATTITUDE \\
\hline EKF & 170.0148 & 7.7596 & 2.1126 \\
\hline UKF & 168.2164 & 7.7612 & 2.0127 \\
\hline AUKF & 163.2533 & 7.4821 & 1.8078 \\
\hline Improvement Over UKF & $2.95 \%$ & $3.60 \%$ & $11.33 \%$ \\
\hline Improvement Over EKF & $3.98 \%$ & $3.58 \%$ & $14.43 \%$ \\
\hline
\end{tabular}

It is observed that, the AUKF algorithm improves the navigation performance by an amount of about 4 percent in the position, 3.5 percent in the velocity and 15 percent in the attitude when compared to the EKF within a scenario including various GPS outages. Due to the selection of the optimal SUT parameters, results of the UKF algorithm are further increased by a small amount. 


\section{CHAPTER 7}

\section{CONCLUSIONS}

In this thesis, the INS/GPS integration is examined and three estimation techniques have been utilized in the context of the study. As a standard and most widely used algorithm, the Extended Kalman Filter is used to solve the regarding estimation problem. In order to improve the performance of the standard INS/GPS integration routine, Unscented Kalman Filter and Adaptive Unscented Kalman Filter implementations are utilized. Designed algorithms are verified and validated as a part of a realistic simulation within the Monte Carlo Analysis. In addition to the simulations, the results are proven with field tests conducted.

Initially, the fundamental information about the inertial navigation systems is presented. The Earth and the gravity models, the error models of the inertial measurement unit, the inertial navigation mechanization equations, and the linear error model of the INS, which is the system model of the designed estimators, are discussed in detail.

After providing the fundamental characteristics of the Global Positioning System, related measurement model and the measurement errors are discussed. A nonlinear measurement model is obatined. The constituted state space and the measurement model for the INS/GPS integration process are used within the estimators which are discussed in detail. 
Finally, the results obtained from the simulations and the field tests are presented and performances of the three estimators are compared in terms of RMSE of the navigation outputs.

It is concluded that UKF provides only a slight improvement in the navigation performance over EKF. When the GPS aiding is obtained regularly, covariance matrices and mean of the state estimate can be kept bounded. In this situation, satisfactory estimation accuracy can be obtained from EKF.

When the GPS outages are considered, accuracy of the standalone navigation deteriorates with the duration of the measurement outage periods. Large GPS outage periods result in large initial state errors and large initial state covariance for the estimators Due to the inaccuracy in the initialization of the estimator states at the end of intermittence of measurements, degradation occurs in the EKF INS/GPS integration in terms of accuracy and convergence time. The underlying reason for this situation is the fact that the first order approximation of the EKF becomes insufficient to cope with the growing nonlinearity [40]. By a proper selection of the parameters of the SUT method, up to fourth order accuracy for any nonlinearity can be obtained from the UKF [32].

One of the most important result of the simulations is that, the performance improvement of the UKF over the EKF increases with increasing initialization errors which means increasing nonlinearity.

When the effects of the SUT parameters on the integrated navigation solution are investigated, it is observed that the performance of the UKF depends on these design parameters. Selection of different parameters for the same navigation scenario results in varying navigation accuracy. Proper designation of the SUT parameters in the context of the AUKF further introduces little increase in the performance of the navigation system. This method is applied by changing the SUT parameters adaptively depending on the recent covariance of the estimator. It is found from the simulations and the field test results that, the correct choice of the SUT parameters at 
specific covariance bounds brings better performance compared to using fixed parameters throughout the scenario.

To further increase the performance of the AUKF, the number of covariance bounds which are defined for specific a SUT parameters can be increased as a feature work. By increasing the test points for finding optimal SUT parameters, these parameters can be selected more accurately.

In the proposed adaptive method, adaptiveness of the selection of the SUT parameters depends on the determinant of the covariance matrix of the position states. Further analysis can be done by using different metrics, such as the trace of the covariance matrix or the filter innovations.

Finally, by making a detailed stochastic observability analysis, further insight can be gained about the AUKF. The effects of the navigation scenario on the performance of the integration algorithm can be observed by considering the observabilities of the individual estimation filter states. 


\section{REFERENCES}

[1] Hofmann Wellenhof, Klaus Legat, Manferd Wieser, Navigation: Principles of Positioning and Guidance, Springer-Verlag Wien New York, 2003

[2] Averil B. Chatfield, Fundamentals of High Accuracy Inertial Navigation, American Institute of Aeronautics and Astronautics, Inc., 1997

[3] Mohinder S. Grewal, L.R. Weill, A.P. Andrews, Global Positioning Systems, Inertial Navigation \& Integration, John Wiley and Sons, New York, 2007

[4] Daniel J. Biezad, Integrated Navigation and Guidance Systems, American Institute of Aeronautics and Astronautics, Inc., 1999

[5] Christopher Jekeli, Integrated Navigation Systems With Geodetic Applications, Walter de Gruyter GmbH \& Co., 2000

[6] Esmat Bekir, Introduction to Modern Navigation Systems, World Scientific Publishing Co. Pte. Ltd., 2007

[7] Robert M. Rogers, Applied Mathematics in Integrated Navigation Systems, American Institute of Aeronautics and Astronautics, Inc., 2007

[8] D.H. Titterton, J.L. Weston, Strapdown Inertial Navigation Technology, American Institute of Aeronautics and Astronautics, 2004

[9] Paul D. Groves, Principles of GNSS, Inertial , and Multisensor Integrated Navigation Systems, Artech House, 2008

[10] NATO RTO-SET-054/RTG-30 Panel, Basic Guide to Advanced Navigation, 2003

[11] George R. Pitman, Inertial Guidance, John Wiley and Sons Inc., New YorkLondon, 1962

[12] Oleg Salychev, Applied Inertial Navigation: Problems and Solutions, The Bauman Moscow State Technical University Press, 2004

[13] George T. Schmidt, "INS/GPS Technology Trends" NATO RTO-SET-116 Panel, 2010 
[14] IEEE Standard Specification Format Guide and Test Procedure for Accelerometers and Gyroscopes, IEEE Std 952\&1293, 1998

[15] National Imagery and Mapping Agency, Department of Defence World Geodetic System 1984, Public Release, 2000

[16] Paul G. Savage, Strapdown Analytics, Strapdown Associates Inc., 2007

[17] Paul G. Savage, "Strapdown Inertial Navigation Integration Algorithm Design Part1: Attitude Algorithms", Journal of Guidance, Control and Dynamics Vol.21, No.1, January-February 1998

[18] Paul G. Savage, "Strapdown Inertial Navigation Integration Algorithm Design Part2: Velocity and Position Algorithms", Journal of Guidance, Control and Dynamics Vol.21, No.1, March-April 1998

[19] Jay A. Farrel, Aided Navigation: GPS with High Rate Sensors, McGrawHill Companies, 2008

[20] Scott Gleason, Demoz Gebre-Egziabher, GNSS Applications and Methods, Artech House Boston-Lodon, 2009

[21] Elliot D. Kaplan, Understanding GPS Principles And Applications, Artech House Inc., 2006

[22] Paul Zarchan, Global Positioning System: Theory and Applications Volume1, American Institute of Aeronautics and Astronautics, Inc., 1996

[23] Kai Borre, A software defined GPS and Galileo receiver, Birk Hauser Boston, 2007

[24] T. Creel, "New, Improved GPS: The Legacy Accuracy Improvement Initiative", GPS World, 2006

[25] George T. Schmidt, Richard E. Philips, "INS/GPS Integration Architectures", NATO RTO-EN-SET-116 Panel, 2010

[26] Santiago Alban, Dennis M. Akos, Stephen M. Rock, "Performance Analysis And Architectures For INS-Aided GPS Tracking Loops”, Department Of Aeronautics And Astronautics, Standford University, 2004

[27] The Navigation Center of Excellence, US Department of Homeland Security, http://www.navcen.uscg.gov , Last access date: 01.12.2011

[28] Robert Grover Brown and Patrick Y.C. Hwang, Introduction to Random Signals and Applied Kalman Filtering. John Wiley and Sons Inc., 1997. 
[29] Mohinder S. Grewal, Angus P. Andrews, Kalman Filtering Theory and Practice Using Matlab, John Wiley and Sons Inc., 2008

[30] Simon Haykin, Kalman Filtering And Neural Networks, John Wiley and Sons Inc, 2001

[31] Junchuan Zhou, Stefan Knedik, Otmar Loffeld, "Development of an Unscented Particle Filter for Tightly Coupled MEMS INS/GPS Integrated Navigation System", ION International Technical Meeting, 2010

[32] Wan E., Van der Merwe, "The Unscented Kalman Filter for Nonlinear Estimation", Proceedings of the IEEE Symposium (AS-SPCC), Lake Louise, Alberta, CA,2000

[33] Simon J. Julier, Jeffrey K. Uhlmann, "Unscented Filtering and Nonlinear Estimation", Proceedings of the IEEE, Vol. 92, No.3, March 2004

[34] Rudolph Van Der Merwe, Arnaud Doucet, Nondo de Freitas, Eric Wan, "The Uncented Particle Filter", Technical Report CUED/F-INFENG/TR 380 Cambridge University Engineering Department, 2000

[35] Yaakov Bar-Shalom, X. Rong Li and Thiagalingam Kirubarajan, Estimation with Applications to Tracking and Navigation. Wiley-Interscience Publication, 2001.

[36] Recep Serdar Acar, Tracking Short Range Ballistic Targets, METU M.Sc. Thesis, 2011

[37] Jae-Hyuk Kim, Sang-Young Park, Young-Rok Kim, “Analysis of Scaling Parameters of the Batch Unscented Transformation for Precision Orbit Determination using Satellite Laser Ranging Data", Journal of Astronomy and Space Sciences, 2011

[38] KVH CNS5000 Continuous Navigation System User Manual, KVH Industries Inc., 2010.

[39] Yong Li, Chris Rizos, Jinlig Wang, Peter Mumford, Weidong Ding, "Sigma Point Kalman Filtering for Tightly Coupled GPS/INS Integration", Navigation: Journal of the Institute of Navigation, Vol.55, No. 3, 2008

[40] Yudan Yi, Dorota A., Grejner-Brzezinska, "Tightly-Coupled GPS/INS Integration Using Unscented Kalman Filter and Particle Filter", ION GNSS $19^{\text {th }}$ International Technical Meeting of the Satellite Division, 26 Sept. 2006 